\title{
Biomarker discovery in heart failure
}

Citation for published version (APA):

Davarzani, N. (2018). Biomarker discovery in heart failure. [Doctoral Thesis, Maastricht University]. Maastricht University. https://doi.org/10.26481/dis.20180702nd

Document status and date:

Published: 01/01/2018

DOI:

10.26481/dis.20180702nd

Document Version:

Publisher's PDF, also known as Version of record

\section{Please check the document version of this publication:}

- A submitted manuscript is the version of the article upon submission and before peer-review. There can be important differences between the submitted version and the official published version of record.

People interested in the research are advised to contact the author for the final version of the publication, or visit the DOI to the publisher's website.

- The final author version and the galley proof are versions of the publication after peer review.

- The final published version features the final layout of the paper including the volume, issue and page numbers.

Link to publication

\footnotetext{
General rights rights.

- You may freely distribute the URL identifying the publication in the public portal. please follow below link for the End User Agreement:

www.umlib.nl/taverne-license

Take down policy

If you believe that this document breaches copyright please contact us at:

repository@maastrichtuniversity.nl

providing details and we will investigate your claim.
}

Copyright and moral rights for the publications made accessible in the public portal are retained by the authors and/or other copyright owners and it is a condition of accessing publications that users recognise and abide by the legal requirements associated with these

- Users may download and print one copy of any publication from the public portal for the purpose of private study or research.

- You may not further distribute the material or use it for any profit-making activity or commercial gain

If the publication is distributed under the terms of Article $25 \mathrm{fa}$ of the Dutch Copyright Act, indicated by the "Taverne" license above, 


\section{BIOMARKER DISCOVERY IN HEART FAILURE}

Naser Davarzani 



\title{
BIOMARKER DISCOVERY IN HEART FAILURE
}

\author{
DISSERTATION
}

to obtain the degree of Doctor at

Maastricht University,

on the authority of the Rector Magnificus,

Prof. dr. Rianne M. Letschert,

in accordance with the decision

of the Board of Deans,

to be defended in public

on Monday 2 July 2018 at 12:00 hours

by

Naser Davarzani 


\section{Supervisors:}

Prof. dr. ir. R. L. M. Peeters

Prof. H. P. Brunner-La Rocca

\section{Co-supervisors:}

Dr. E. N. Smirnov

Dr. J. M. H. Karel

\section{Assessment Committee:}

Prof. dr. G. B. Weiss (chair)

Prof. dr. I. C. W. Arts

Prof. dr. G. Molenberghs (Hasselt University)

Prof. dr. C. E. Müller (University Hospital Basel)

Dr. V.P. M. van Empel

\section{1) Maastricht University universiteit

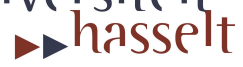

This research was funded in part by the transnational University Limburg (tUL).

2 Dissertation Series No. 2018-15

The research reported in this thesis has been carried out under the auspices of SIKS, the Dutch Research School for Information and Knowledge Systems.

ISBN 978-94-6233-994-1

(C) Naser Davarzani, 2018.

All rights reserved. No part of this publication may be reproduced, stored in a retrieval system, or transmitted, in any form or by any means, electronically, mechanically, photocopying, recording or otherwise, without prior permission of the author. 


\section{Contents}

Contents $\diamond 1$

1 Introduction $\diamond 3$

1.1 Conceptual Framework $\diamond 5$

1.2 Objectives of the thesis $\diamond 6$

1.3 Structure of the Thesis $\diamond 9$

2 Background $\diamond 11$

2.1 The TIME-CHF Study $\diamond 12$

2.2 Modelling Repeated Events $\diamond 16$

2.3 Bayesian Inference $\diamond 18$

2.4 Logistic-GEE Models $\diamond 22$

3 NT-proBNP-Guided Therapy Reduces Repeated Heart Failure Hospitalizations $\diamond 25$

3.1 Introduction $\diamond 27$

3.2 Methods $\diamond 28$

3.3 Results $\diamond 29$

3.4 Discussion $\diamond 33$

3.5 Conclusions $\diamond 36$

4 Cutpoint Estimation for Continuous Covariates Dichotomization $\diamond 37$

4.1 Introduction $\diamond 39$

4.2 Dichotomization Using Linear Regression Models $\diamond 41$

4.3 Simulation study $\diamond 44$

4.4 Application to the TIME-CHF Study $\diamond 49$

4.5 Discussion $\diamond 52$

5 Generalized Ranking Accuracy for Logistic-GEE Models $\diamond 54$

5.1 Introduction $\diamond 56$

5.2 Generalized Ranking Accuracy $\diamond 59$

5.3 Simulation study $\diamond 65$

5.4 Application to the TIME-CHF Study $\diamond 75$

5.5 Conclusion $\diamond 78$ 
6 Novel Concept to Guide Systolic Heart Failure Medication by Repeated Biomarker Testing $\diamond 80$

6.1 Introduction $\diamond 82$

6.2 Methods $\diamond 83$

6.3 Results $\diamond 86$

6.4 Discussion $\diamond 90$

7 Conclusions $\diamond 95$

7.1 Answer to the Research Questions $\diamond 96$

7.2 Directions for Future Research $\diamond 99$

References $\diamond 102$

Publication List $\diamond 118$

Summary $\diamond 120$

Valorization $\diamond 123$

Acknowledgements $\diamond 125$

About the Author $\diamond 127$

SIKS Dissertation Series $\diamond 128$ 


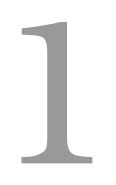

\section{Introduction}


Adequately utilized, data in the medical field can be an inexhaustible source of knowledge to fuel a learning health care system and help clinicians in making the right decision. The design of data analysis methods in medical studies is changing fundamentally due to the complexities generated in medical data, such as having too many variables or missing data and more specifically, having repeated correlated measures in time. Therefore, the need to develop new methods to address questions that can deal with these complexities is emerging. However, the issue with newly developed methods may be the lack of fully-fledged validation of these techniques that does not enable us be certain about the obtained results and to fully know how to extend the obtained results to future data.

Heart failure (HF) is recognised as a frequent disease in Western countries that causes increasing public health problems and is the topic of many clinical studies. HF is the main cause of hospital admission among elderly in developed countries [51,83] and it is very likely that prevalence will further increase in most countries [50,72]. Regardless of the main causes of hospitalization, these admissions impose enormous health care costs related to relatively long hospital stays [81, 127]. Therefore, precisely diagnosing and monitoring progression of disease in HF patients may have a major role in reducing disease burden and health care costs. Currently, diagnosing HF and monitoring patients are mainly based on clinical signs and symptoms. However, management of patients based on clinical signs and symptoms can be challenging as signs and symptoms of HF are not specific [111]. Moreover, monitoring progression of the disease can be very challenging and it is often impossible to select individuals who are most likely to need hospitalization, or to progress to mortality, and thus who are in need of more intensive treatment. Therefore, new tools are required to better monitor patients and to define the most effective treatment. Biomarkers are promising in this regard, as ideally, they predict outcome quite well. Moreover, some, e.g., Brain Natriuretic Peptide (BNP) and N-terminal pro-B-type Natriuretic Peptide (NT-proBNP), have shown to be of diagnostic value in HF [37, 146]. Due to the fact that biomarkers reflect different aspect of disease and state of body, they may not only have diagnostic and prognostic usages, but also therapeutic implications. In order to move towards individualized therapy, implementing biomarkers to guide management of HF can help select patients in need of more intensive therapy and improve therapeutic decision making. So far, however, only very few biomarkers are being used in daily practice due to the lack of direct therapeutic consequences based on biomarker measurement.

This thesis investigates the diagnostic and prognostic role of biomarkers and their usages in establishing a guidance in medical HF therapy in individual HF patients. The analyses are based on data feom the Trial of Intensified versus standard Medical therapy in Elderly patients with Congestive Heart Failure (TIME-CHF) [19, 102] study, as it contains multiple biomarker measurements over time together with detailed information on medication, allowing one to investigate the interaction between biomarkers and treatment 
effects.

\subsection{Conceptual Framework}

\subsubsection{Definition of Heart Failure}

Heart failure (HF), sometimes referred to as Congestive Heart Failure (CHF), occurs when the heart muscle does not pump sufficient blood to fulfill the requirements of the body as well as it should. In a clinical definition, HF is a clinical syndrome with according symptoms and signs, such as edema (accumulation of fluid causing swelling in the leg), dyspnea (shortness of breath, excessive tiredness), fatigue, together with proof of an underlying functional and/or structural hearth disease [84, 85].

Problems of HF increase with age [116] and is the most important reason for hospitalization in patients aged $\geq 65$ years $[83,122]$. HF has a high prevalence such that $1 \%$ to $2 \%$ of the population and up to $10 \%$ of the population aged $\geq 70$ years suffer from CHF $[90,110]$. HF hospitalizations have a great impact on disease burden, particularly quality of life, and on health care costs [81].

The diagnosis of HF is difficult, especially when relying on signs and symptoms only $[111,85]$. In addition to physical examinations, diagnostic tests support the diagnosis of HF, e.g., chest X-ray laboratory tests, electrocardiography, and echocardiography. However, diagnosing HF remains difficult, especially in elderly patients. This is due to the fact that in older age, HF patients usually have multiple comorbidities such as renal failure, diabetes, pulmonary disorders, cancer, anemia, cerebrovascular and peripheral arterial occlusive disease. In addition to comorbidities, in elderly patients various other factors such as sedentary lifestyle may mitigate the signs of HF. Hence investigating the relationship between other markers, e.g. biomarkers, and the severity and diagnosis of HF seems necessary and helpful, especially in elderly patients.

\subsubsection{Biomarkers in Heart Failure}

Biomarkers usually refer to measurable circulating markers in the blood that may indicate some biological processes in the body. Biomarkers are usually enzymes or proteins that are in relationship with one or more pathophysiological pathways relevant in HF [5, 115, 120]. Evaluating the diagnostic and prognostic accuracy and effectiveness of biomarkers in HF has been a topic of great interest in the last decades [58, 115, 137].

Assessing the relationship between biomarkers and disease not only can be of diagnostic and prognostic value, but also may have therapeutic implications $[4,86]$. Knowing 
about the prognostic implications of biomarkers might be interesting, but without having therapeutic consequences, they may not be of clinical use.

Despite the large amount of research related to biomarkers, the use of biomarkers in the treatment of HF is still very limited. Among the biomarkers investigated, BNP and NT-proBNP are the only ones that currently are incorporated in the guidelines for diagnosis of HF [133]. It has been shown that the circulating level of NT-proBNP is normally very low in healthy individuals and is increased in HF, and correlates well with ventricular wall stress and severity of $\operatorname{HF}[58,80]$. Most of the medical HF therapies significantly reduce NT-proBNP levels [37, 146]. Therefore, NT-proBNP has been also suggested as a tool to tailor and intensify medical HF therapy. Several trials and metaanalyses suggested that performing repeated measurements of NT-proBNP may help to establish guideline recommended medical HF therapy in HF with reduced ejection fraction (HFrEF) patients and to improve outcome [65, 96].

\subsubsection{Heart Failure Medications}

Monitoring progression of HF is not that a easy task since it is also mainly based on interpreting the clinical symptoms and signs. Therefore it remains difficult to predict and prevent HF events accurately and consequently it is very difficult or even sometimes impossible to find the best drug therapy for individual HF patients.

In the past decades, medical therapy for HFrEF has evolved greatly [88]. Several medication classes - e.g., Renin-Angiotensin System (RAS) blockers encompassing AngiotensinConverting-Enzyme (ACE)-inhibitors and Angiotensin-Receptor Blockers (ARBs), $\beta$ blockers and Mineralocorticoid Receptor Antagonists (MRAs) - have proven to improve prognosis in HFrEF and therefore are recommended in high doses by current guidelines [145]. In addition, (loop) diuretics are recommended for decongestion to relieve symptoms [145]. However, in clinical practice, it is often difficult to implement all medication classes and to reach guideline recommended dosage, especially in elderly and comorbid patients [78]. In such cases, it would be crucial to know which drug is most important to be given in high doses to improve prognosis, and which may be less important. At present, it is largely impossible to make this decision for an individual patient.

\subsection{Objectives of the thesis}

This thesis has two main objectives: a medical objective and a methodological objective.

The medical objective of this thesis is to investigate biomarkers in the TIME-CHF study as markers to monitor progression of HF and to guide HF drug therapy. The methodological objective of the thesis is to develop new methods with the aim to facilitate 
investigation of biomarkers, or other variables, and to develop criteria backing the results covering the medical objective of the thesis.

We deal with these main objectives by studying the following research questions:

Research Question 1: Does NT-proBNP guided therapy reduce repeated HF hospitalizations?

HF therapy guided by NT-proBNP may be superior to standard therapy in patients with chronic HF as shown in various meta-analyses [36, 104, 121]. However, the obtained results from different individual trials were not uniform $[69,102,133]$. This is most likely because of limited power of these trials due to a relatively small sample size.

The TIME-CHF study is the largest trial evaluating NT-proBNP-guided therapy so far in the HF domain. The results have shown that there was no significant effect on the primary endpoint hospital-free survival, while the disease specific endpoint HF hospitalfree survival was significantly improved $[102,118]$.

Despite the fact that repeated hospitalizations (both related to HF and other causes $[29,51])$ are very common in HF patients, most intervention trials for treatment of HF have only evaluated the effect on time-to-first event (hospitalization or death). Since hospitalizations are common (and a very costly problem) [81, 127], it may be clinically more relevant to see whether any new HF therapy prevents hospitalizations beyond the first event $[17,113]$.

Research Question 2: What is the best cutpoint for dichotomization of continuous variables?

Dichotomizing a continuous variable is a common part of many diagnostic and prognostic analyses, such as biomarker studies, and is widely used in clinical research. Dichotomization of a covariate makes it easier for clinicians to interpret the covariate's effect on the outcome variable and guides them in their choice of therapy. By using dichotomized covariates we may have a simpler interpretation of common effect measures from statistical models such as odds ratios and hazard ratios. They can also help to avoid errors of wrong functionality form assumptions for covariates in regression models, and to make data summarization more efficient. Therefore, selecting or estimating a cutpoint for a covariate might be worthwhile in many directions.

Research Question 3: How to measure the goodness-of-fit and the predictive perform- 
ance of a logistic-GEE model?

In many clinical trials such as TIME-CHF, observations for individual patients are often repeated over time (longitudinal data). The difficulty with any investigation in such a trial lies within the fact that the outcomes for a single patient, which could be the quality of life of a patient or the risk of hospitalization at several points of time, are likely to be correlated. Moreover, repeated measures of biomarkers, medication and other covariates are typically correlated as well. Therefore, the correlation between outcomes and variation of covariates in time needs to be taken into account for proper analysis.

Among the methods dealing with correlated observations, logistic Generalized Estimating Equations (logistic-GEE) has become the most popular regression model for analyzing longitudinal data with binary outcomes [47, 73]. However, assessing the goodness-offit and predictive performance of logistic-GEE models is problematic, due to the fact that the outcomes are correlated within a patient and no likelihood is available. So far, several criteria and tests have been proposed for assessing the goodness-of-fit of logistic-GEE models in the literature (see e.g, [31, 32, 107]). However, most of them have shortcomings, making it impossible to have a commonly applicable and accepted criterion or test. Moreover, to the best of our knowledge there is no criterion reflecting the predictive performance of logistic-GEE models. Therefore, in order to have a better interpretation of the obtained results, using a logistic-GEE model and its extension to a future data set, we need a measure that can reflect both goodness-of-fit and predictive performance of the model.

Research Question 4: Can biomarkers predict treatment effects of medication in patients with systolic HF?

Repeated measurements of NT-proBNP, as was suggested in several studies, may help to establish medical therapy as considered by the guidelines in HFrEF patients $[65,96]$. However, guiding HF therapy by a single marker is a limited approach because one biomarker cannot cover the complex pathophysiological pathways that play a role in HF. In fact, the NT-proBNP approach is based on the idea that patients at highest risk of poor outcome are in need of intensified therapy. However, there is no specific tailoring of individual therapy. In addition, despite a general beneficial effect of HF drugs, not every drug may have the same benefit in a specific patient, or for a patient subgroup. Furthermore, different medication classes in HF interfere with different pathways. Therefore, a combination of biomarkers that reflect these pathways may be better to indicate which medication class is most important to up-titrate or possibly also to down-titrate in a spe- 
cific patient, aiming towards precision medicine in HF. Unfortunately at present, it is still impossible to make this decision for an individual patient.

\subsection{Structure of the Thesis}

These four research questions have shaped the structure of this thesis. Chapter 2 provides the reader with general background information on the TIME-CHF study and the methods used to address the research questions. In order to address research question 1 , in Chapter 3, we have employed the gap-time method (proposed by Prentice, Williams and Peterson $[10,106])$ to investigate the effect of NT-proBNP-guided therapy on the recurrence of all-cause hospitalization events (all-cause hospitalization or death) and HF hospitalization events (HF hospitalization or death) in TIME-CHF. We do this not only for all patients, but also for different age groups. In Chapter 4, we deal with research question 2. To this end we propose a Bayesian approach for dichotomizing continuous covariates when using a regression model. This method can be used both in univariate and in multivariate settings. We have also applied the proposed Bayesian approach to estimate the cutpoint for some covariates in TIME-CHF. In Chapter 5, we address research question 3 and propose a new measure for evaluating the quality of logistic-GEE models, namely Generalized Ranking Accuracy (GRA). GRA can be used for assessing the goodness-of-fit and the predictive performance of logistic-GEE models depending on the type of assessment method (e.g. cross-validation). In this chapter we also compare the GRA with some other existing criteria and assess the performance of GRA in a simulation study. In Chapter 6, we deal with research question 4 and explore the hypothesis that biomarkers may be able to predict response to therapy in HF in TIME-CHF. We investigate the interaction between multiple repeatedly measured biomarkers, and the response to the four most important classes of $\mathrm{HF}$ medication regarding the risk of $\mathrm{HF}$ hospitalization or death. To this end we employ logistic-GEE models and implement the GRA to evaluate these models and to assess the reliability of the obtained results. Finally, in Chapter 7, we conclude the thesis by answering the research questions and providing an outlook on possible directions for future research. 



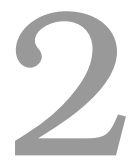

Background 
This chapter provides general background information about the Trial of Intensified versus standard Medical therapy in Elderly patients with Congestive Heart Failure (TIME$\mathrm{CHF}$ ). It also discusses the methods we used to address research questions 1-4. In Section 2.1 we introduce the TIME-CHF study and give information about the characteristics of the patients, available biomarkers, and medications. In Section 2.2 we present the Prentice, Williams and Peterson Gap-Time (PWP-GT) model as a method for analysing repeated events which helps us to deal with research question 1, in Chapter 3. In Section 2.3 we give general information about Bayesian Inference that we use to address research question 2, in Chapter 4. In Section 2.4 we briefly explain and formalize the bipartite ranking task for clustered data and the logistic Generalized Estimation Equations (GEE) model. The latter we use when dealing with research questions 3 and 4, in Chapters 5 and 6 , respectively.

\subsection{The TIME-CHF Study}

The TIME-CHF study was a multicenter trial that involved 15 centers in Switzerland and Germany [19, 102]. The study included 622 patients aged 60 years or older with symptomatic Heart Failure (HF), HF hospitalization within the last year, and a N-terminal pro-B-type Natriuretic Peptide (NT-proBNP) level of $400 \mathrm{pg} / \mathrm{mL}$ or higher in patients younger than 75 years and a level of $800 \mathrm{pg} / \mathrm{mL}$ or higher in patients aged 75 years or older. Patients were randomized to two treatment groups: standard (symptom-guided) therapy or intensified (NT-proBNP-guided) therapy during the 18 months of the trial. Both treatment groups were stratified into two age groups of 60 to 74 and 75 years or older. In this single-blind trial, patients in contrast with treating physicians were blinded to treatment group allocation.

According to the protocol used, after the baseline assessment, the patients were treated and followed up for 18 months in pre-specified visits after 1, 3, 6, 12, and 18 months, with further follow-up up to 5.5 years. For each patient, every hospitalization together with its cause and eventual mortality were recorded. The median of the overall survival time (time until death) was 3.96 (range from 0.01 to 5.5) years and $60.3 \%$ of patients were alive after 5.5 years follow-up.

Within 5.5 years follow-up, 132 patients (61 NT-proBNP-guided, 71 symptom-guided) experienced one and 345 patients (167 NT-proBNP-guided, 178 symptom-guided) two or more all-cause hospitalization events (all-cause hospitalization or death). Regarding HF hospitalization events (HF hospitalization or death), 166 patients (78 NT-proBNP-guided, 88 symptom-guided) experienced one event and 151 patients (68 NT-proBNP-guided, 83 symptom-guided) two or more events. The median number of all-cause hospitalization events and HF hospitalization events were 2 and 1, respectively. 
Three main types of covariates were measured per patient in the TIME-CHF study:

Patient characteristics: Table 2.1 presents the baseline characteristics of patients we used in this thesis, per treatment group as mean \pm standard deviation for continuous variables or as numbers and percentages for binary variables.

Biomarkers: Blood samples were drawn at every visit; samples were stored at -80 degrees Celsius until analysis. At the end of the trial, twenty biomarkers were measured from these stored samples of all available visits. The selection of biomarkers was based on the representation of different pathways that are known to reflect important pathophysiological pathways [120]. The following biomarkers were measured: Soluble FMS-Like Tyrosine kinase-1 (SFLT), Growth Differentiation Factor 15 (GDF15), CYStatin-C (CYSC), Ferritin, Interleukin-6 (IL6), Placental Like Growth Factor (PLGF), Sex Hormone-Binding Globulin (SHBG), Soluble TransFerrin Receptor (STFR), High Sensitivity Troponin T (HSTNT), Type-1 Procollagen N-terminal Peptide (TP1NP), Uric acid (Uric), Blood Urea Nitrogen (BUN), Soluble ST2 (SST2), N-terminal pro-B-type Natriuretic Peptide (NT-proBNP), Creatinine, High Sensitivity C-Reactive Protein (HSCRP), Prealbumin (PREA), Osteopontin (OPN), Mimican, and Insulin-like Growth Factor-Binding Protein 7 (IGFBP7). The assays used to measure these markers are summarized in Table 2.2.

HF medications: The medication doses for the four most important HF drug classes: $\beta$ blockers, Renin-Angiotensin System (RAS) blockers, Spironolactone and Loop diuretics were available on a daily basis. Doses of $\beta$-blockers and RAS blockers are expressed as the percentage of the target dose as reported in [87] (e.g. 5mg of Ramipril per day is $50 \%$ of the target dose of $10 \mathrm{mg} / \mathrm{day}$ ). For combinations of Angiotensin-ConvertingEnzyme (ACE)-inhibition and Angiotensin-Receptor Blockers (ARB), the relative doses were added and expressed as a combined dose. Spironolactone is given in $\mathrm{mg}$ as it was the only Mineralocorticoid Receptor Antagonist (MRA) used in TIME-CHF. Loop diuretics are expressed as the equivalent dose of furosemide (i.e. $40 \mathrm{mg}$ of furosemide $=10 \mathrm{mg}$ of torasemide $=1 \mathrm{mg}$ of bumetanide). 


\begin{tabular}{cccc}
\hline Patient characteristics & $\begin{array}{c}\text { Symptom-guided } \\
(\mathrm{n}=312)\end{array}$ & $\begin{array}{c}\text { NT-proBNP-guided } \\
(\mathrm{n}=310)\end{array}$ & P-value \\
\hline Age (years) & $77.3 \pm 7.7$ & $76.6 \pm 7.4$ & 0.247 \\
Male sex (\%) & $177(56.7)$ & $192(61.9)$ & 0.951 \\
Ischemic HF etiology $(\%)$ & $171(54.8)$ & $159(51.3)$ & 0.379 \\
Charlson Score & $3.1 \pm 1.7$ & $3.2 \pm 1.7$ & 0.638 \\
Kidney disease $(\%)$ & $175(56.1)$ & $180(58.1)$ & 0.619 \\
Diabetes $(\%)$ & $121(38.8)$ & $101(32.6)$ & 0.107 \\
Hypertension $(\%)$ & $236(75.6)$ & $226(72.9)$ & 0.435 \\
Stroke $(\%)$ & $29(9.3)$ & $23(7.4)$ & 0.398 \\
Cancer $(\%)$ & $43(13.8)$ & $43(13.9)$ & 0.974 \\
Anemia $(\%)$ & $85(27.2)$ & $90(29)$ & 0.620 \\
NYHA $>$ II $(\%)$ & $238(76.3)$ & $235(75.8)$ & 0.889 \\
Rales $(\%)$ & $146(46.8)$ & $134(43.5)$ & 0.411 \\
Angina $(\%)$ & $62(20.1)$ & $64(20.8)$ & 0.842 \\
COPD $(\%)$ & $57(18.3)$ & $67(26.1)$ & 0.297 \\
LVEF $(\%)$ & $35.1 \pm 13.2$ & $34.8 \pm 12.7$ & 0.785 \\
Heart rate & $76 \pm 14.4$ & $75.1 \pm 13.9$ & 0.359 \\
Body mass index $\left(\mathrm{kg} / \mathrm{m}^{2}\right)$ & $25.5 \pm 4.7$ & $25.7 \pm 4.1$ & 0.959 \\
Systolic blood pressure $(\mathrm{mmHg})$ & $122.3 \pm 21$ & $121.5 \pm 19.4$ & 0.781 \\
Hemoglobin $(\mathrm{g} / \mathrm{l})$ & $129.6 \pm 18$ & $131.4 \pm 18.2$ & 0.165 \\
Potassium $(\mathrm{mmol} / \mathrm{l})$ & $4.1 \pm 0.49$ & $4.1 \pm 0.55$ & 0.986 \\
\hline
\end{tabular}

Table 2.1: Patient characteristics at the baseline visit in the TIME-CHF study. The variables are given for the Symptom-guided therapy and the NT-proBNP-guided therapy groups. P-value indicates the difference for variables with respect to patients from the Symptom-guided and the NT-proBNP-guided groups (the Mann-Whitney U-test for continuous variables and the $\chi^{2}$ test for binary variables).

NYHA: New York Heart Association functional classification; LVEF: Left Ventricular Ejection Fraction; COPD: Chronic Obstructive Pulmonary Disease. 


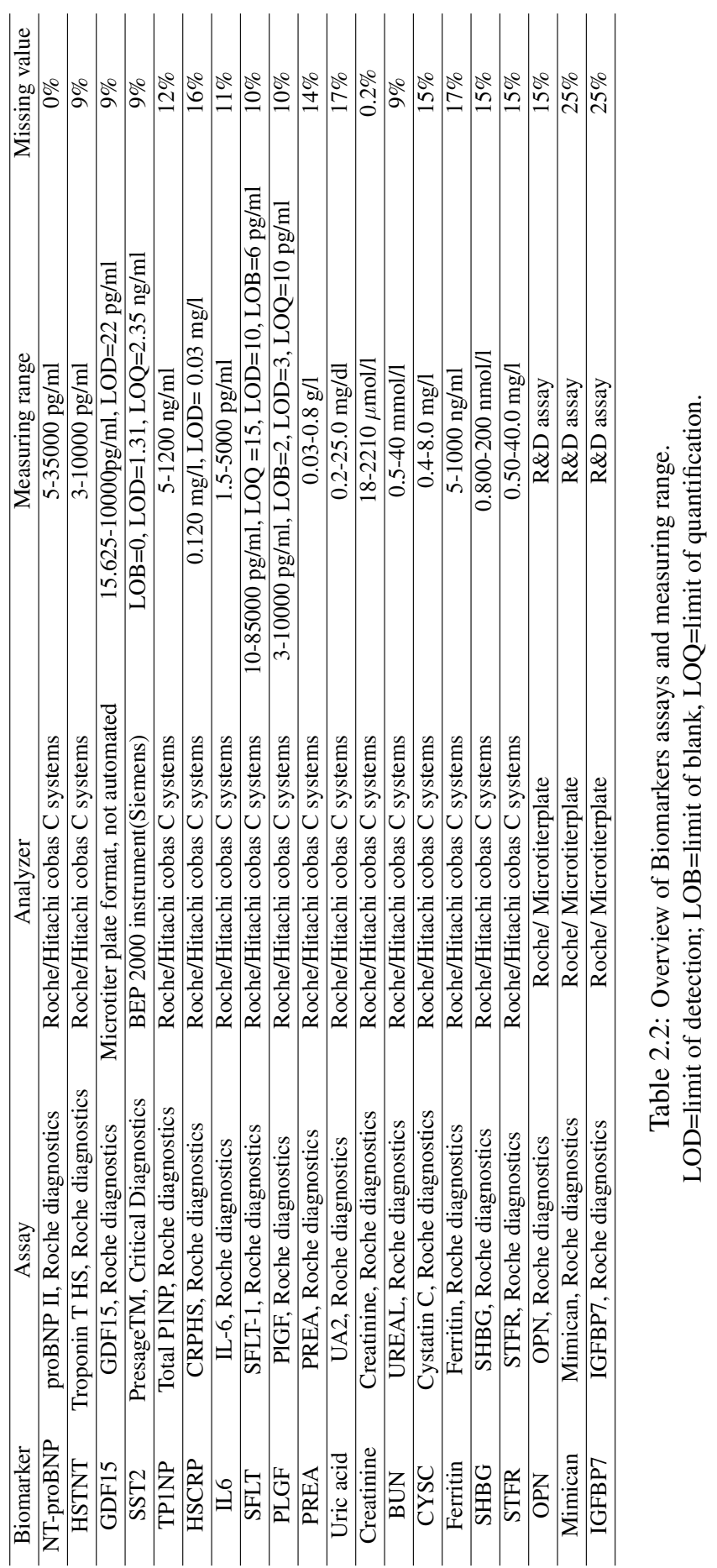




\subsection{Modelling Repeated Events}

In many medical studies, an event of interest may occur twice or more often for the same patient during the follow up period. However, most of the survival analysis methods investigate only the first time-to-event and ignore repeated events [17, 113]. Examples of repeated events data in heart disease clinical trials include: repeated hospitalisations in HF patients, recurrent heart attacks of coronary patients, or relapses of cardiovascular diseases.

Repeated events are likely to be correlated because they belong to the same patient. For example a patient with an HF hospitalization is more prone to experience another HF hospitalization or die. Hence, to correctly analyze repeated events, the correlated nature of the data needs to be taken into account. Several models have been proposed for analysing repeated events $[10,28,54,63,106]$. The most commonly used methods so far analyze time to repeated events (either time from the start of a study to each event, or time between repeated events) or simply the number of repeated events. Amongst the latter such approaches, both Poisson and negative binomial regression are commonly used for modelling the number of events $[43,55,114]$. However, the methods which take time between repeated events into account, use different models.

The survival based model of Andersen-Gill (AG) [11] is an approach for analyzing repeated events. The AG model, as a generalization of the Cox proportional hazards model, is formulated in terms of Gap-Times (times between successive events) for the same subject. The use of a robust variance estimator is recommended with the AG model to adjust for the correlation among outcomes of the same subject [74]. However, a shortcoming of the AG model is that it cannot take into account the order of events.

A more appropriate model to analyze repeated events is the Prentice, Williams and Peterson model [10, 106] based on the Gap-Times (PWP-GT model). The PWP-GT model is not only able to take into account the dependence of events within a subject, but also the order of events. Below we explain how the PWP-GT model works.

Let $N(t)$ be the number of events a subject has experienced by time $t$ and $\boldsymbol{X}(t)$ the vector of covariates of the subject at time t. For a subject with $S$ events, let $t_{s}$ be the $s^{\text {th }}$ recurrence time, $s=1, \ldots, S$, let $t_{0}=0$ and let $t_{S+1}$ be the time of censoring. The censoring may occur because a subject has not experienced the event before the end of the study or has withdrawn from the study. For a subject the $s^{t h}$ gap-time and the corresponding covariate vector at time $t_{s}$ are assigned to the stratum $s$. A subject moves to stratum $(s+1)$ immediately after his $s^{\text {th }}$ recurrence time and remains there until the $(s+1)^{\text {st }}$ recurrence occurs or until censor time. We note that PWP-GT does not use the information in stratum $S+1$, since there is no event in this stratum. The PWP-GT model 
is a stratified Cox-type model of the form:

$$
\lambda(t \mid N(t), \boldsymbol{X}(t))=\lambda_{0 s}\left(t-t_{s-1}\right) e^{\boldsymbol{\beta}_{s}^{\prime} \boldsymbol{X}(t)}, t_{s-1} \leq t<t_{s}, s=1, \ldots, S,
$$

where $\lambda_{0 s} \geq 0$ is an arbitrary baseline hazard function (depending on the Gap-Times distribution), and $\boldsymbol{\beta}_{s}$ is a vector of stratum-specific regression coefficients.

To illustrate the data layout for applying PWP-GT models, suppose subject $i$ experiences the $s^{t h}$ event at time $t_{s i}$. Then the gap-time $u_{s i}=t_{s i}-t_{s-1, i}$ is the time duration between the $(s-1)^{\text {st }}$ and $s^{\text {th }}$ events for the subject in stratum $s$. Assume that there are $n$ subjects with at most $S$ observed events, then the data layout is as illustrated in Table 2.3. Note that in Table 2.3 the order of subject is regardless of the number of events.

\begin{tabular}{cccccc}
\hline Subject ID & Start-Time & Stop-Time & Gap-Time & Stratum & Status* $^{*}$ \\
\hline 1 & 0 & $t_{11}$ & $u_{11}$ & 1 & 1 \\
\hline 1 & $t_{11}$ & $t_{21}$ & $u_{21}$ & 2 & 1 \\
\hline 1 & $t_{21}$ & $t_{31}$ & $u_{31}$ & 3 & 0 \\
\hline 2 & 0 & $t_{12}$ & $u_{12}$ & 1 & 1 \\
\hline 2 & $t_{12}$ & $t_{22}$ & $u_{22}$ & 2 & 0 \\
\hline$\vdots$ & $\vdots$ & $\vdots$ & $\vdots$ & $\vdots$ & $\vdots$ \\
\hline$k$ & 0 & $t_{1 k}$ & $u_{1 k}$ & 1 & 1 \\
\hline$k$ & $t_{1 k}$ & $t_{2 k}$ & $u_{2 k}$ & 2 & 1 \\
\hline$\vdots$ & $\vdots$ & $\vdots$ & $\vdots$ & $\vdots$ & \\
\hline$k$ & $t_{S-1, k}$ & $t_{S k}$ & $u_{S k}$ & $S$ & 1 \\
\hline$k$ & $t_{S k}$ & $t_{S+1, k}$ & $u_{S+1, k}$ & $S+1$ & 0 \\
\hline$\vdots$ & $\vdots$ & $\vdots$ & $\vdots$ & $\vdots$ & \\
\hline$n$ & 0 & $t_{1 n}$ & $u_{1 n}$ & 1 & 1 \\
\hline$n$ & $t_{1 n}$ & $t_{2 n}$ & $u_{2 n}$ & 2 & 0 \\
\hline
\end{tabular}

Table 2.3: Layout of dataset for applying PWP-GT models. *Event status in the given stratum ( $1=$ event and $0=$ censoring).

Now consider the estimation of stratum-specific regression coefficients $\boldsymbol{\beta}_{s}$ in model (2.1). To achieve this we should obtain the partial likelihood of the regression coefficients.

To indicate the order of gap-times in a stratum, we define the notation $u_{(s j)}$ as the $j^{\text {th }}$ smallest gap-time in stratum $s$. For example, suppose that four gap-times are recorded in stratum $s$, e.g., $u_{s 1}=5, u_{s 2}=8 u_{s 3}=6 u_{s 4}=2$. The ordered gap-times will be denoted by $u_{(s 1)}=2, u_{(s 2)}=5, u_{(s 3)}=6$ and $u_{(s 4)}=8$. 
Let $u_{(s 1)}<\cdots<u_{\left(s k_{s}\right)}$ denote the ordered gap-times for the $k_{s}$ events occurring in stratum $s, u_{(s 0)}=0$ and $u_{\left(s, k_{s}+1\right)}=\infty$, such that the subject with the $j^{\text {th }}$ ordered gap-time $u_{(s j)}$ experiences the event at time $t_{s j}$ and the $\boldsymbol{X}_{s j}\left(t_{s j}\right)$ be the corresponding vector of covariates, $j=1, \ldots, k_{s}$. Let $R(u, s)$ be the set of subjects at risk in stratum $s$ at gap-time $u \in\left[u_{(s, i-1)}, u_{(s i)}\right), i=1, \ldots, k_{s}+1$. Under the above assumptions, the partial likelihood $L(\boldsymbol{\beta})$ can be written as:

$$
L(\boldsymbol{\beta})=\prod_{s \geq 1} \prod_{j=1}^{k_{s}} \frac{\exp \left\{\boldsymbol{\beta}_{s} \boldsymbol{X}_{s j}\left(t_{s j}\right)\right\}}{\sum_{l \in R\left(u_{(s j)}, s\right)} \exp \left\{\boldsymbol{\beta}_{s} \boldsymbol{X}_{l}\left(t_{s-1, l}+u_{(s j)}\right)\right\}},
$$

where, $\boldsymbol{\beta}=\left(\boldsymbol{\beta}_{1}, \ldots, \boldsymbol{\beta}_{S}\right)^{\prime}, \boldsymbol{X}_{l}(t)$ is the vector of covariates for the $l^{t h}$ subject at time $t$. We note that in PWP-GT modeling one may apply robust sandwich variance estimators $[74,147]$ to estimate the variances of the estimated regression coefficients when we have correlated observations from the same patient.

\subsection{Bayesian Inference}

Statistical inference is a collection of procedures with the aim of extracting information from available data [20]. The Bayesian approach is an approach of statistical inference which combines prior information about unknown parameters with the observations at hand to reach updated (posterior) conclusions about the parameters [71].

Let $\boldsymbol{Y}=\left(Y_{1}, \ldots, Y_{n}\right)$ be samples of $n$ i.i.d. outcomes (discrete or continuous) such that $f_{Y \mid \Theta}\left(y_{i} \mid \boldsymbol{\theta}\right)$ presents a probability function or a density function of $Y_{i}$ given a vector of continuous parameters $\boldsymbol{\theta} \in D_{\theta}, i=1, \ldots, n$. Then the likelihood function of $\boldsymbol{\theta}, L(\boldsymbol{\theta} \mid \boldsymbol{y})$, is defined as the joint distribution of $Y_{1}, \ldots, Y_{n}$, given by $L(\boldsymbol{\theta} \mid \boldsymbol{y})=$ $f_{\boldsymbol{Y} \mid \boldsymbol{\Theta}}(\boldsymbol{y} \mid \boldsymbol{\theta})=\prod_{i=1}^{n} f_{Y \mid \Theta}\left(y_{i} \mid \boldsymbol{\theta}\right)$.

Assume that $\pi(\boldsymbol{\theta})$ is the prior distribution function for $\boldsymbol{\theta}$, which presents the information about parameter $\boldsymbol{\theta}$ irrespective of any data that might become available. Then the distribution $f_{Y, \Theta}(\boldsymbol{y}, \boldsymbol{\theta})$ can be split up into $f_{Y, \Theta}(\boldsymbol{y}, \boldsymbol{\theta})=f_{Y \mid \Theta}(\boldsymbol{y} \mid \boldsymbol{\theta}) \pi(\boldsymbol{\theta})$. Following Bayes' rule [71] the posterior distribution function for $\boldsymbol{\theta}$ is given by:

$$
\begin{aligned}
\pi(\boldsymbol{\theta} \mid \boldsymbol{y}) & =\frac{L(\boldsymbol{\theta} \mid \boldsymbol{y}) \pi(\boldsymbol{\theta})}{f_{\boldsymbol{Y}}(\boldsymbol{y})} \\
& =\frac{L(\boldsymbol{\theta} \mid \boldsymbol{y}) \pi(\boldsymbol{\theta})}{\int_{D_{\theta}} L(\boldsymbol{\theta} \mid \boldsymbol{y}) \pi(\boldsymbol{\theta}) d \boldsymbol{\theta}} .
\end{aligned}
$$

Expression (2.3) shows that the posterior distribution of $\boldsymbol{\theta}$, given the data $\boldsymbol{y}$, is propor- 
tional to the product of the likelihood function of $\boldsymbol{\theta}$ with its prior distribution, i.e.

$$
\pi(\boldsymbol{\theta} \mid \boldsymbol{y}) \propto L(\boldsymbol{\theta} \mid \boldsymbol{y}) \pi(\boldsymbol{\theta})
$$

since the denominator depends only on the observed data $\boldsymbol{y}$ which is assumed to be fixed in the Bayesian context.

\subsubsection{Bayes Estimator}

Suppose $\boldsymbol{Y}=\left(Y_{1}, \ldots, Y_{n}\right)$ are $n$ i.i.d. samples from distribution $f_{Y \mid \Theta}(\boldsymbol{y} \mid \boldsymbol{\theta})$. Let $\pi(\boldsymbol{\theta})$ be the prior distribution and $\hat{\boldsymbol{\theta}}$ an estimator of $\boldsymbol{\theta}$ and define $D(\boldsymbol{\theta}, \hat{\boldsymbol{\theta}})$ as a loss function. Then the Bayes estimator is defined as the estimator that minimizes the expected loss $E(D(\boldsymbol{\theta}, \hat{\boldsymbol{\theta}}) \mid \boldsymbol{y})$ over the posterior distribution $\pi(\boldsymbol{\theta} \mid \boldsymbol{y})$, among all estimators [20].

Theorem 2.1: The Bayes estimator of $\boldsymbol{\theta}$ under the squared error loss function, i.e., $D(\boldsymbol{\theta}, \hat{\boldsymbol{\theta}})=(\boldsymbol{\theta}-\hat{\boldsymbol{\theta}})^{2}$, is the mean of the posterior distribution [20]:

$$
\hat{\boldsymbol{\theta}}=E(\boldsymbol{\theta} \mid \boldsymbol{y})=\int_{D_{\theta}} \boldsymbol{\theta} \pi(\boldsymbol{\theta} \mid \boldsymbol{y}) d \boldsymbol{\theta}
$$

\subsubsection{Posterior Equal Tail Credible Interval}

Having the posterior distribution $\pi(\theta \mid \boldsymbol{y})$, for a scalar parameter $\theta$, let us denote the posterior cumulative distribution function as $F(\theta)$. Then $[a, b]$ is defined as a $100(1-\alpha) \%$ credible interval for $\theta$ when

$$
\operatorname{Pr}(a<\theta \leq b \mid \boldsymbol{y})=1-\alpha=F(b)-F(a) .
$$

In this case, the $100(1-\alpha) \%$ equal tail credible interval can be obtained by calculating $a$ and $b$ such that: $\operatorname{Pr}(\theta \leq a \mid \boldsymbol{y}) \equiv F(a)=\alpha / 2$ and $\operatorname{Pr}(\theta \geq b \mid \boldsymbol{y}) \equiv 1-F(b)=\alpha / 2$.

\subsubsection{Markov Chain Monte Carlo Methods}

When performing Bayesian inference to obtain the Bayes estimates or any properties of posterior distributions we need to calculate integrals as in (2.5). Unfortunately, this often requires calculating analytically intractable integrals (not expressible in elementary functions). In such cases, instead of solving the analytical equations, we may use classical numerical integration methods, but we may also resort to using sampling techniques based upon Markov Chain Monte Carlo (MCMC) methods [40].

Monte Carlo integration can be used to approximate properties of posterior distributions required for a Bayesian analysis. For an arbitrary function of $\boldsymbol{\theta}, h(\boldsymbol{\theta})$, consider the 
integral:

$$
\int_{D_{\theta}} h(\boldsymbol{\theta}) \pi(\boldsymbol{\theta} \mid \boldsymbol{y}) d \boldsymbol{\theta}
$$

If we generate a large number $\boldsymbol{\theta}^{(1)}, \ldots, \boldsymbol{\theta}^{(n)}$ of random values from the posterior distribution $\pi(\boldsymbol{\theta} \mid \boldsymbol{y})$, then:

$$
\int_{D_{\theta}} h(\boldsymbol{\theta}) \pi(\boldsymbol{\theta} \mid \boldsymbol{y}) d \boldsymbol{\theta}=E(h(\boldsymbol{\theta}) \mid \boldsymbol{y}) \simeq \frac{1}{n} \sum_{i=1}^{n} h\left(\boldsymbol{\theta}^{(i)}\right) .
$$

By choosing $n$ sufficiently large, the right hand side value in (2.8) is known to converge to that of the integral on the left hand side one aims to compute; the variance of the estimate on the right hand side can also be tracked to obtain information on the accuracy of this approximation. This is referred to as Monte Carlo integration [40].

Often the posterior distribution for $\boldsymbol{\theta}$ has no known probability distribution form to easily generate the samples. It may also happen that we even cannot obtain the posterior distribution in a fully closed form and we may only know that it is proportional to the product of the likelihood function and the prior distribution as in (2.4). Therefore, one problem with Monte Carlo integration is in obtaining samples $\boldsymbol{\theta}^{(1)}, \ldots, \boldsymbol{\theta}^{(n)}$ from $\pi(\boldsymbol{\theta} \mid \boldsymbol{y})$ to approximate the integral in (2.8). This problem can be solved using MCMC methods, which are methods for sampling from probability distributions using Markov chains. A Markov chain is a sequence of samples that transitions from one sample to another in a probabilistic fashion such that the next sample only depends on the current sample. By taking enough steps, the distribution underlying a Markov chain converges to the stationary distribution and the samples can be used as samples of the desired distribution. In this chapter, we introduce Metropolis-Hastings (M-H) [23] and the Gibbs sampling method [38] as two widely used MCMC methods in the literature.

\section{The Metropolis-Hastings Algorithm}

Suppose our goal is to draw samples from some posterior distribution $\pi(\boldsymbol{\theta} \mid \boldsymbol{y})$ such that $\pi(\boldsymbol{\theta} \mid \boldsymbol{y}) \propto L(\boldsymbol{\theta} \mid \boldsymbol{y}) \pi(\boldsymbol{\theta})$. Define $p(\boldsymbol{\theta})=L(\boldsymbol{\theta} \mid \boldsymbol{y}) \pi(\boldsymbol{\theta})$, then the Metropolis-Hastings (M-H) algorithm generates $n$ samples from $\pi(\boldsymbol{\theta} \mid \boldsymbol{y})$, through the following steps [23]:

1. Take any initial $\boldsymbol{\theta}^{(0)}$ value, satisfying $p\left(\boldsymbol{\theta}^{(0)}\right)>0$, and set $t=1$.

2. Sample a candidate point $\boldsymbol{\theta}^{*}$ from an arbitrary distribution $q\left(\boldsymbol{\theta}^{*} \mid \boldsymbol{\theta}^{(t-1)}\right)$, which is known as the proposal distribution, such that $\boldsymbol{\theta}^{*}$ has the same domain as $\boldsymbol{\theta}$.

3. Set $\alpha$ equal to $\min \left(\frac{p\left(\boldsymbol{\theta}^{*}\right) q\left(\boldsymbol{\theta}^{(t-1)} \mid \boldsymbol{\theta}^{*}\right)}{p\left(\boldsymbol{\theta}^{(t-1)}\right) q\left(\boldsymbol{\theta}^{*} \mid \boldsymbol{\theta}^{(t-1)}\right)}, 1\right)$. 
4. Generate $u$ from a uniform ditribution $\mathrm{U}(0,1)$.

5. If $\alpha>u$ then (the candidate is accepted)

$$
\text { set } \boldsymbol{\theta}^{(t)}=\boldsymbol{\theta}^{*} \text {. }
$$

else (the candidate is rejected)

$$
\text { set } \boldsymbol{\theta}^{(t)}=\boldsymbol{\theta}^{(t-1)} \text {. }
$$

6. If $t<n$ then

$$
\text { set } t=t+1 \text { and go to step } 2 \text {. }
$$

else

stop.

The above M-H algorithm generates a Markov chain $\left(\boldsymbol{\theta}^{(0)}, \boldsymbol{\theta}^{(1)}, \ldots, \boldsymbol{\theta}^{(n)}\right)$. Usually it is necessary to throw away sufficient initial samples, say $k$, as a burn-in period that the chain approaches to the stationary state. Then samples from the vector $\left(\boldsymbol{\theta}^{(k)}, \boldsymbol{\theta}^{(k+1)}, \ldots, \boldsymbol{\theta}^{(n)}\right)$ are used as samples from $\pi(\boldsymbol{\theta} \mid \boldsymbol{y})$.

The advantage of the $\mathrm{M}-\mathrm{H}$ algorithm is that by using a proposal distribution we can indirectly sample from the desired distribution when it is too complex to directly sample from. The disadvantage, of course, is that the $\mathrm{M}-\mathrm{H}$ algorithm as an accept-reject algorithm is computationally expensive when we need to sample multiple parameters.

\section{Gibbs Sampling Method}

For the benefit of the reader, and to give a basic understanding of Gibbs sampling, we shall first consider it in a two dimensional set up. Gibbs sampling, in a two-dimensional set up, provides a method to generate samples from the joint posterior distribution $\pi\left(\theta_{1}, \theta_{2} \mid \boldsymbol{y}\right)$ when direct sampling is difficult. The generated samples can be used to approximate the joint posterior distribution or the marginal posterior distributions $\pi\left(\theta_{1} \mid \boldsymbol{y}\right)$ and $\pi\left(\theta_{2} \mid \boldsymbol{y}\right)$ [38]. This approach is based on the property that the joint posterior distribution $\pi\left(\theta_{1}, \theta_{2} \mid \boldsymbol{y}\right)$ can be uniquely determined from $\pi\left(\theta_{1} \mid \theta_{2}, \boldsymbol{y}\right)$ and $\pi\left(\theta_{2} \mid \theta_{1}, \boldsymbol{y}\right)$. The Gibbs sampling starts by taking initial values for the parameters, say $\theta_{1}^{(0)}$ and $\theta_{2}^{(0)}$ in the range of $\theta_{1}$ and $\theta_{2}$, respectively. Then it generates $\theta_{1}^{(t)}$ and $\theta_{2}^{(t)}(t=1,2,3, \ldots)$ in a sequential manner such that for the given $\theta_{1}^{(t)}$ and $\theta_{2}^{(t)}$ at iteration $t$, the $(t+1)^{\text {st }}$ values for the parameters are generated through the following iterative scheme:

- Sample $\theta_{1}^{(t+1)}$ from $\pi\left(\theta_{1} \mid \theta_{2}^{(t)}, \boldsymbol{y}\right)$

- Sample $\theta_{2}^{(t+1)}$ from $\pi\left(\theta_{2} \mid \theta_{1}^{(t+1)}, \boldsymbol{y}\right)$

We note that Gibbs sampling method is a special case of $\mathrm{M}-\mathrm{H}$ sampling in which the candidate value is always accepted (i.e. $\alpha=1$ ) [23]. 
Gibbs sampling with $n$ iterations generates a sequence of values $\theta_{1}^{(1)}, \theta_{2}^{(1)}, \theta_{1}^{(2)}, \theta_{2}^{(2)}$ $, \ldots, \theta_{1}^{(n)}, \theta_{2}^{(n)}$. After a sufficient burn-in period the samples can be used to approximate properties of the posterior distributions $\pi\left(\theta_{1}, \theta_{2} \mid \boldsymbol{y}\right), \pi\left(\theta_{1} \mid \boldsymbol{y}\right)$ and $\pi\left(\theta_{2} \mid \boldsymbol{y}\right)$.

Assuming a multivariate posterior distribution $\pi(\boldsymbol{\theta} \mid \boldsymbol{y}), \boldsymbol{\theta}=\left(\theta_{1}, \ldots, \theta_{p}\right)$, Gibbs sampling works according to the following iterative scheme. Given the initial value $\boldsymbol{\theta}^{(0)}=\left(\theta_{1}^{(0)}, \ldots, \theta_{p}^{(0)}\right)$, at iteration $(t+1)$ the sampling procedure performs the following $p$ steps:

- Sample $\theta_{1}^{(t+1)}$ from $\pi\left(\theta_{1} \mid \theta_{2}^{(t)}, \ldots, \theta_{p}^{(t)}, \boldsymbol{y}\right)$

- Sample $\theta_{2}^{(t+1)}$ from $\pi\left(\theta_{2} \mid \theta_{1}^{(t+1)}, \theta_{3}^{(t)}, \ldots, \theta_{p}^{(t)}, \boldsymbol{y}\right)$

- Sample $\theta_{p}^{(t+1)}$ from $\pi\left(\theta_{p} \mid \theta_{1}^{(t+1)}, \ldots, \theta_{p-1}^{(t+1)}, \boldsymbol{y}\right)$

Gibbs sampling is easy to implement for multivariate distributions and does not depend much on the initial values. Moreover there is no need to tune any proposal distribution. However, the disadvantage of Gibbs sampling method is that we must be able to derive the conditional distributions. Also, the conditional distributions should be relatively easy to sample from.

\subsection{Logistic-GEE Models}

Clustered data are very common in biomedical, clinical, and social sciences research $[6,68,131]$. Clustered data are defined as data with a clustered or grouped structure. A cluster (group) can consist of variable measurements of related subjects or repeated variable measurements for a single subject such that in either case the measurements may correlate. For example, in the social sciences, members of the same household form a cluster, since they are more likely to give rise to similar measurements than nonmembers. In clinical trials, it is often the case that the observations for each subject are repeated over time (longitudinal data). Since these observations are likely to be correlated, they naturally form a cluster. In order to correctly analyze clustered data, the correlation within clusters needs to be taken into account. However, this correlation may often be unknown and there may be insufficient data to estimate such correlations accurately for each group.

In this thesis we study clustered data when the outcome variables are binary. A usual model for analyzing data with binary outcomes is logistic regression. However, due to the correlation between the observations (within clusters), such models cannot be directly used to analyze clustered data. Therefore, Liang and Zeger in [73, 147] introduced Generalized Estimating Equations (GEEs), as an extension of the quasi-likelihood approach [141] to estimate the parameters of a logistic regression model with a possible unknown correlation between the outcomes or covariates. When we use GEEs to estimate the model 
parameters of a logistic regression model applied to clustered data, this is known as a logistic-GEE model. Logistic-GEE models the relationship between the covariates and the probability of a positive outcome (outcome $=1$ ) on the population level, while there are unknown correlations between outcomes within a cluster.

\subsubsection{Bipartite Ranking and Logistic-GEE Models}

The bipartite ranking task assumes that we have $n$ subjects. The $i^{\text {th }}$ subject is represented by a cluster of $m_{i}$ observations such that the $t^{\text {th }}$ observation, $t=1, \ldots, m_{i}$ is given with $p$ covariates $X_{i t 1}, \ldots, X_{i t p}$ in $\mathbb{R}$ and a binary outcome variable $Y_{i t}$. Hence, the $i^{\text {th }}$ cluster is identified by $\left\langle\boldsymbol{X}_{i}, \boldsymbol{Y}_{i}\right\rangle$, where $\boldsymbol{X}_{i}=\left(\boldsymbol{X}_{i 1}, \ldots, \boldsymbol{X}_{i m_{i}}\right)^{\prime}$ in which $\boldsymbol{X}_{i t}=\left(X_{i t 1}, \ldots, X_{i t p}\right)^{\prime}$ is a $p \times 1$ vector of covariates for observation $t$ for subject $i$ and $\boldsymbol{Y}_{i}=\left(Y_{i 1}, \ldots, Y_{i m_{i}}\right)^{\prime}$ is an $m_{i} \times 1$ vector of binary outcomes. For different clusters, $i$ and $j$, we assume that the correlation between $\boldsymbol{Y}_{i}$ and $\boldsymbol{Y}_{j}$ equals zero but correlation between $Y_{i s}$ and $Y_{i k}$ may be nonzero, $s \neq k$, and the covariates may be either fixed or changing at every cluster level.

Given $n$ clusters $\left\langle\boldsymbol{X}_{i}, \boldsymbol{Y}_{i}\right\rangle, i=1, \ldots, n$, the task of bipartite ranking is to find an ordering on the instances such that positive instances (outcome=1) are ranked higher than negative ones (outcome $=0$ ). To solve the task we try to find a real-valued ranking function that maps any covariate $\boldsymbol{X}_{i t}, i=1, \ldots, n, t=1, \ldots, m_{i}$, to a real number. The number has to be high if covariate $\boldsymbol{X}_{i t}$ has a positive outcome $\left(Y_{i t}=1\right)$; otherwise, it has to be low. The ranking function can be used to induce an ordering over the covariates $\boldsymbol{X}_{i t}$, $i=1, \ldots, n, t=1, \ldots, m_{i}$, to reflect their relationship with respect to positive outcome.

Logistic-GEE models solve the bipartite ranking task, since they essentially provide a ranking function for clustered data. A logistic-GEE model explains the outcome variable $Y_{i t}, i=1, \ldots, n, t=1, \ldots, m_{i}$, from the covariate $\boldsymbol{X}_{i t}$ using a ranking function of the form:

$$
\log \left(\frac{\pi_{i t}}{1-\pi_{i t}}\right)=\beta_{0}+\boldsymbol{\beta} \boldsymbol{X}_{i t}, i=1, \ldots, n, t=1, \ldots, m_{i},
$$

where, $\pi_{i t}=\mathrm{E}\left(Y_{i t} \mid \boldsymbol{x}_{i t}\right)=\operatorname{Pr}\left(Y_{i t}=1 \mid \boldsymbol{x}_{i t}\right)$ for $t=1, \ldots, m_{i}, \beta_{0}$ is the population averaged intercept term and $\boldsymbol{\beta}=\left(\beta_{1}, \ldots, \beta_{p}\right)$ is the vector of population averaged coefficients.

The logistic-GEE model can be obtained by estimating the unknown regression coefficient vector $\gamma=\left(\beta_{0}, \boldsymbol{\beta}\right)$. Estimating the coefficients can be done by solving the following generalized estimating equations [73]:

$$
\sum_{i=1}^{n}\left(\frac{\partial \boldsymbol{\pi}_{i}}{\partial \gamma}\right)^{\prime} \boldsymbol{W}_{i}^{-1}\left(\boldsymbol{y}_{i}-\boldsymbol{\pi}_{i}\right)=\mathbf{0}
$$

where $\boldsymbol{\pi}_{i}=\left(\pi_{i 1}, \ldots, \pi_{i m_{i}}\right)^{\prime}$ for $i=1, \ldots, n, \boldsymbol{W}_{i}=\boldsymbol{A}_{i}{ }^{1 / 2} \boldsymbol{R}_{i}(\boldsymbol{\alpha}) \boldsymbol{A}_{i}{ }^{1 / 2}$ is the working covariance matrix for $\boldsymbol{Y}_{\boldsymbol{i}}$ and $\boldsymbol{A}_{\boldsymbol{i}}$ is a diagonal matrix, $\operatorname{diag}\left[\operatorname{var}\left(Y_{i 1}\right), \ldots, \operatorname{var}\left(Y_{i m_{i}}\right)\right]$, 
such that $\operatorname{var}\left(Y_{i m_{i}}\right)$, the variance of $Y_{i m_{i}}$, equals to $\pi_{i m_{i}}\left(1-\pi_{i m_{i}}\right) . \boldsymbol{R}_{i}(\boldsymbol{\alpha})$ is the $m_{i} \times m_{i}$ working correlation matrix for $\boldsymbol{Y}_{\boldsymbol{i}}$, where $\boldsymbol{\alpha}$ may be an arbitrary $h \times 1$ vector of some unknown parameters involved in the working correlation structure.

We note that the working correlation matrix $\boldsymbol{R}_{i}(\boldsymbol{\alpha})$ can be chosen in different ways depending on the nature of the correlation between outcomes $Y_{i s}$ and $Y_{i k}, s \neq k,[21,57]$. For instance, assuming a cluster of size 4 , the correlations between the outcomes of the cluster may have the following structures:

- Exchangeable: if the observations from a subject have no time dependence. In this case one may hypothesize that the outcomes within a cluster have a single common correlation, that is, $\boldsymbol{\alpha}$ is a scalar, such that:

$$
\operatorname{Corr}\left(Y_{i s}, Y_{i k}\right)=\left\{\begin{array}{lll}
1 & \text { if } & s=k \\
\alpha & \text { if } & s \neq k,
\end{array}\right.
$$

which results in a correlation matrix of the form:

$$
\boldsymbol{R}_{i}(\boldsymbol{\alpha})=\left[\begin{array}{cccc}
1 & \alpha & \alpha & \alpha \\
\alpha & 1 & \alpha & \alpha \\
\alpha & \alpha & 1 & \alpha \\
\alpha & \alpha & \alpha & 1
\end{array}\right]
$$

- Autoregressive (order one): if we assume a time dependence for the observations from a subject. In this case the correlation structure may the assumed to be $\operatorname{Corr}\left(Y_{i s}, Y_{i k}\right)=$ $\alpha^{|s-k|}$. This results in a correlation matrix of the form:

$$
\boldsymbol{R}_{i}(\boldsymbol{\alpha})=\left[\begin{array}{cccc}
1 & \alpha & \alpha^{2} & \alpha^{3} \\
\alpha & 1 & \alpha & \alpha^{2} \\
\alpha^{2} & \alpha & 1 & \alpha \\
\alpha^{3} & \alpha^{2} & \alpha & 1
\end{array}\right]
$$

Note that one may also adopt an Autoregressive correlation structure with a higher order. For more examples of correlation structures and their motivations, see [48]. We note that an attractive property of a logistic-GEE model is that the resulting estimation of regression coefficients of the model is consistent and asymptotically normal even when the correlation structure within the clusters is imprecisely specified [73]. 


\section{NT-proBNP-Guided Therapy Reduces Repeated Heart Failure Hospitalizations}

This chapter is based on: Davarzani, N., Sanders-van Wijk, S., Karel, J., Maeder, M.T., Leibundgut, G., Gutmann, M., Pfisterer, M.E., Rickenbacher P., Peeters, R., Brunner-La Rocca, H.P. N-terminal pro-b-type natriuretic peptide-guided therapy reduces repeated hospitalizations - results from TIME-CHF. Journal of Cardiac Failure, 2017, 23(5):382389. 


\begin{abstract}
Background: Although heart failure (HF) patients are known to experience repeated hospitalizations, most studies only evaluated time-to-first event. N-terminal pro-B-type Natriuretic Peptide (NT-proBNP)-guided therapy may improve HF-specific outcome, but effects on recurrence of all-cause hospitalization are uncertain. Therefore, we investigated the effect of NT-proBNP-guided therapy on repeated events in HF, using a time-betweenevents approach in a hypothesis generating analysis.
\end{abstract}

Methods and Results: TIME-CHF randomized $499 \mathrm{HF}$ patients, aged $\geq 60$ years, $\mathrm{LVEF} \leq$ $45 \%$, NYHA $\geq$ II to NT-proBNP-guided versus a symptom-guided therapy for 18 months, with further follow-up for 5.5 years. The effect of NT-proBNP-guided therapy on repeated HF-related and all-cause hospitalizations and all-cause death was explored. One hundred four patients (49 NT-proBNP-guided, 55 symptom-guided) experienced one and 275 patients (133 NT-proBNP-guided, 142 symptom-guided) two or more all-cause hospitalization events. Regarding HF hospitalization events, 132 patients (57 NT-proBNPguided, 75 symptom-guided) experienced one and 122 patients (57 NT-proBNP-guided, 65 symptom-guided) two or more events. NT-proBNP-guided therapy was significant in preventing second all-cause hospitalizations (Hazard Ratio $(\mathrm{HR})=0.83, \mathrm{P}=0.01$ ) in contrast to non-significant results in preventing first all-cause hospitalization events $(H R=0.91$, $\mathrm{P}=0.35)$. This was not the case regarding $\mathrm{HF}$ hospitalization events $(\mathrm{HR}=0.85, \mathrm{P}=0.14$ vs. $\mathrm{HR}=0.73, \mathrm{P}=0.01$ ). The beneficial effect of NT-proBNP-guided therapy was only seen in patients aged $<75$ years, but not in those aged $\geq 75$.

Conclusion: NT-proBNP-guided therapy reduces the risk of recurrent events in patients aged $<75$ years. This included all-cause hospitalization by mainly reducing later events, adding knowledge to the neutral effect on this endpoint when shown using time-to-first event analysis only. 


\subsection{Introduction}

Although it is well known that heart failure (HF) patients suffer from repeated hospitalizations (both related to HF and other causes $[29,51]$ ), most intervention trials for treatment of HF have only evaluated the effect on time-to-first event. Since hospitalizations have a great impact on disease burden, particularly quality of life, and on health care costs $[81,127]$, it may be clinically more relevant to see whether any new HF therapy prevents hospitalizations beyond the first event, i.e. repeated events [17, 113]. However, methods to investigate repeated events have not yet been widely established and such studies are relatively scarce. The most commonly used methods so far, analyzed the number of days alive outside the hospital or simply the number of repeated events. Among the latter approach, the Poisson and negative binomial regressions are commonly used to compare hospitalization (or other events) rates in different groups [43, 55, 114]. However these methods do not take into account the time between repeated events. The Poisson distribution ignores intra-individual correlation of events within a patient, although the recurrence of hospitalizations and consecutive death of a patient are correlated [41, 143]. Alternatively, modeling the gap times between successive events (hospitalizations or death) as random outcomes is an approach to investigate the treatment effect on recurrence of events $[10,28,54,63,106]$.

Heart failure therapy guided by N-terminal pro-B-type Natriuretic Peptide (NT-proBNP) may be superior to standard therapy in patients with chronic HF as shown in various metaanalyses [36, 104, 121]. However, results from individual trials have not been uniform $[69,102,133]$. Various reasons may be responsible for this, including limited power of these trials due to a relatively small sample size. The largest trial evaluating NT-proBNPguided management so far, i.e. the Trial of Intensified versus standard Medical therapy in Elderly patients with Congestive Heart Failure (TIME-CHF)[19], was a neutral study, because no significant effect on the primary endpoint hospital-free survival was found, while the disease specific endpoint HF hospital-free survival was significantly improved $[102,118]$. As there was no safety problem present [119], positive effects might have been concealed by non-cardiac events unrelated to the intervention, particularly because in patients aged $>75$ years significant comorbidities reduced the benefit of HF therapy on global hospitalization/death outcomes [102, 118]. Analysis of repeated events analysis might reveal effects on the primary endpoint not seen by time-to-first event analysis. Therefore in this chapter we employ the Prentice, Williams and Peterson Gap-Times model (PWP-GT) [10, 106] to investigate the effect of NT-proBNP-guided therapy on the recurrence of all-cause hospitalization events (all-cause hospitalization or death) and HF hospitalization events (HF hospitalization or death) in TIME-CHF study. The PWP-GT model is introduced in detail in Section 2.2. 


\subsection{Methods}

\subsubsection{TIME-CHF Study and Design}

The design and results of the TIME-CHF study have been described in detail in Section 2.1. The study in this chapter includes 499 patients aged 60 years or older with symptomatic HF, Left Ventricular Ejection Fraction (LVEF) $<45 \%$ and New York Heart Association (NYHA) $\geq$ II from the TIME-CHF study. Patients were randomized to intensified, NT-proBNP-guided versus a standard, symptom-guided therapy for 18 months, with further follow-up for 5.5 years. Both treatment groups were stratified into two age groups of 60 to 74 years and 75 years or older. For each patient, time to recurrence of all-cause hospitalizations, HF hospitalizations and mortality were recorded, for 5.5 years.

\subsubsection{Statistical Methods}

Baseline characteristics are presented as mean \pm standard deviation (SD) for continuous normally distributed variables, median and quartiles for non-normally distributed continuous variables, or as numbers and percentages for categorical variables, see Tables 3.1 and 3.2 .

Differences in the baseline characteristics per number of HF hospitalization events and all-cause hospitalization events (none vs. one, none vs. at least one, one vs. two or more), recorded within 5.5 years follow-up, were assessed using a t-test for continuous normally distributed variables, a Mann-Whitney U-test for non-normally distributed continuous variables, and a $\chi^{2}$-test for categorical variables. All tests were two-sided at a 5 percent level of significance and adjusted for multiple comparisons. The time between successive hospitalizations was calculated (time-interval) for all-cause and HF-hospitalizations and mortality within 5.5 years follow-up. The outcome variable was censored at the time of last follow-up if a patient did not experience an event.

The effect of NT-proBNP-guided versus symptom-guided therapy was assessed using the PWP-GT model [106]. The PWP-GT model formulation and usages are explained in Section 2.2. It can be used to explore the effects based on the time between successive events, using stratified Cox models. In PWP-GT, time intervals between repeated events are outcomes of interest. Patients are not restricted to have the same number of events, so depending on the number of repeated events, patients may have a different number of outcomes. For each patient, the first measured outcome is the time from baseline until the onset of first event (hospitalization or death). The second outcome (for patients with at least one hospitalization during the study) is the time from the onset of the first hospitalization until the onset of the second event, and so forth for patients with more than two events. In the likelihood formulation, all the patients are at risk of an event for 
the first stratum, but only those who experienced hospitalization in the previous stratum are at risk for a successive event. The approach considers the order in which events occur and measures the effect of treatment on each consecutive event.

In this study, we considered the gap-time analysis only up to the second HF hospitalization events and third all-cause hospitalization events. All analyses were performed with SAS (Version 9.2, SAS Institute Inc., Cary., NC).

(1) All-cause Hospitalizations

(2) Heart Failure Hospitalizations

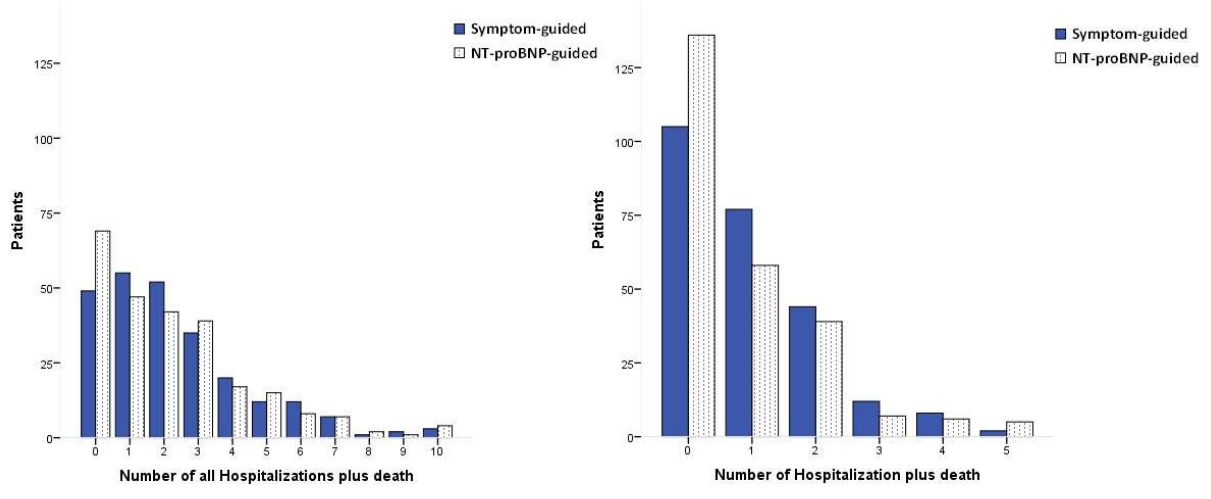

Figure 3.1: The frequency of (1) all-cause hospitalizations (including death), (2) HF hospitalizations (including death) for Symptom-guided and NT-proBNP-guided patients.

\subsection{Results}

\subsubsection{Frequency of events and baseline characteristics}

The frequency of hospitalization events, within 5.5 years follow-up, for NT-proBNPguided and symptom-guided patients is presented in Figure 3.1. 104 patients (49 NTproBNP-guided, 55 symptom-guided) experienced one and 275 patients (133 NT-proBNPguided, 142 symptom-guided) two or more all-cause hospitalization events. Regarding HF hospitalization events, 132 patients (57 NT-proBNP-guided, 75 symptom-guided) experienced one and 122 patients (57 NT-proBNP-guided, 65 symptom-guided) two or more events. The median number of hospitalizations events was 2 for both groups, and there was no significant difference between the two groups regarding the total number of events. Among the patients without any event, the prevalence of patients randomized to NT-proBNP-guided therapy was higher than the prevalence of patients randomized to symptom-guided therapy, whereas this was the opposite for patients with one and two 
events. Baseline characteristics of patients with a different number of all-cause hospitalization events and HF hospitalization events are presented in Table 3.1 and Table 3.2, respectively.

In comparison to patients without any event, those with one or more all-cause hospitalization event were older and more likely to suffer from coronary artery disease, kidney disease, diabetes and, also a higher Charlson comorbidity score (Table 3.1). Moreover, they had more severe symptoms, higher NT-proBNP and creatinine and lower hemoglobin plasma concentrations at baseline. Interestingly, there were no significant differences between patients with more than one versus those with just one event. A comparable pattern is seen when considering HF hospitalization events as illustrated in Table 3.2.

\begin{tabular}{ccccccc}
\hline & \multicolumn{2}{c}{ All-cause hospitalization events } & & & \\
& None & One & Two or more & $\dagger \mathrm{P}_{01}$ & ${ }^{\ddagger} \mathrm{P}_{0}$ & $\S \mathrm{P}_{01}$ \\
& $(\mathrm{n}=120)$ & $(\mathrm{n}=104)$ & $(\mathrm{n}=275)$ & & & \\
\hline Age (years) & $73.9 \pm 7.3$ & $75.3 \pm 7.5$ & $77.3 \pm 7.3$ & 0.153 & 0.000 & 0.018 \\
Male sex (\%) & $66(55)$ & $79(76)$ & $182(66)$ & 0.001 & 0.005 & 0.066 \\
Ischemic HF etiology (\%) & $44(37)$ & $60(57)$ & $183(66)$ & 0.002 & 0.000 & 0.108 \\
Charlson Score, median [IQR] & $2[1-2]$ & $3[2-4]$ & $3[2-5]$ & 0.000 & 0.000 & 0.103 \\
Kidney disease (\%) & $48(40)$ & $62(60)$ & $167(61)$ & 0.003 & 0.000 & 0.843 \\
Diabetes (\%) & $29(24)$ & $35(34)$ & $108(39)$ & 0.014 & 0.006 & 0.314 \\
Hypertension (\%) & $81(67)$ & $76(75)$ & $198(72)$ & 0.363 & 0.312 & 0.834 \\
Stroke (\%) & $4(0.3)$ & $11(1.0)$ & $26(9)$ & 0.030 & 0.025 & 0.742 \\
Cancer (\%) & $16(13)$ & $18(17)$ & $34(12)$ & 0.408 & 0.914 & 0.211 \\
Anemia (\%) & $13(10)$ & $23(22)$ & $96(35)$ & 0.021 & 0.000 & 0.016 \\
NYHA $>$ II (\%) & $87(72)$ & $77(74)$ & $207(75)$ & 0.795 & 0.594 & 0.804 \\
Rales (\%) & $38(31)$ & $40(38)$ & $131(48)$ & 0.287 & 0.009 & 0.109 \\
Angina (\%) & $18(15)$ & $20(19)$ & $69(25)$ & 0.400 & 0.048 & 0.229 \\
LVEF (\%) & $3226[1760-5590]$ & $4592[2067-8085]$ & $4670[2658-8069]$ & 0.021 & 0.004 & 0.982 \\
NT-proBNP (ng/l), median [IQR] & $29.5 \pm 7.5$ & $29.42 \pm 7.8$ & $30 \pm 7.8$ & 0.921 & 0.695 & 0.522 \\
Body mass index (kg/m $\left.{ }^{2}\right)$ & $25.1 \pm 4.7$ & $25.5 \pm 4.6$ & $25.2 \pm 3.9$ & 0.502 & 0.634 & 0.545 \\
Systolic blood pressure (mmHg) & $119 \pm 18$ & $116 \pm 19$ & $118 \pm 17$ & 0.248 & 0.431 & 0.226 \\
Hemoglobin (g/l) & $136 \pm 20$ & $132 \pm 20$ & $130 \pm 18$ & 0.104 & 0.004 & 0.383 \\
Creatinine (mmol/l) & $100 \pm 30$ & $119 \pm 37$ & $123 \pm 39$ & 0.000 & 0.000 & 0.351 \\
Potassium (mmol/l) & $4.0 \pm 0.4$ & $4.0 \pm 0.5$ & $4.1 \pm 0.5$ & 0.615 & 0.195 & 0.019 \\
\hline
\end{tabular}

Table 3.1: Baseline characteristics according to number of all-cause hospitalizations plus death. ${ }^{\dagger} \mathrm{P}_{01}$ : p-value comparison between those patients not hospitalized and those hospitalized only once (two sided t-test or Mann-Whitney U-test for continuous variables and $\chi^{2}$ test for categorical variables).

${ }^{\ddagger} \mathrm{P}_{0}$ : p-value comparison between those patients not hospitalized and those hospitalized at least once.

${ }^{\S} \mathrm{P}_{01}$ : p-value comparison between those patients hospitalized once and those hospitalized two times or more.

IQR: Interquartile range. 


\begin{tabular}{|c|c|c|c|c|c|c|}
\hline & \multicolumn{3}{|c|}{ HF hospitalization events } & \multirow[b]{2}{*}{${ }^{\dagger} \mathrm{P}_{01}$} & \multirow[b]{2}{*}{${ }^{\ddagger} \mathrm{P}_{0}$} & \multirow[b]{2}{*}{${ }^{\S} \mathrm{P}_{01}$} \\
\hline & $\begin{array}{c}\text { None } \\
(\mathrm{n}=245)\end{array}$ & $\begin{array}{c}\text { One } \\
(\mathrm{n}=132)\end{array}$ & $\begin{array}{c}\text { Two or more } \\
\quad(\mathrm{n}=122)\end{array}$ & & & \\
\hline Age (years) & $74.1 \pm 7.4$ & $78.1 \pm 7$ & $77.9 \pm 7.2$ & 0.000 & 0.000 & 0.850 \\
\hline Male sex (\%) & $149(60)$ & $96(72)$ & $82(67)$ & 0.020 & 0.0295 & 0.337 \\
\hline Ischemic HF etiology (\%) & $109(44)$ & $88(35)$ & $90(73)$ & 0.000 & 0.000 & 0.216 \\
\hline Charlson Score, median [IQR] & $2[1-4]$ & $3[2-5]$ & $3[2-5]$ & 0.000 & 0.0001 & 0.831 \\
\hline Kidney disease (\%) & $113(46)$ & $80(60)$ & $84(68)$ & 0.007 & 0.0001 & 0.169 \\
\hline Diabetes (\%) & $65(26)$ & $57(43)$ & $50(40)$ & 0.001 & 0.000 & 0.722 \\
\hline Hypertension (\%) & $170(69)$ & $99(75)$ & $86(70)$ & 0.250 & 0.395 & 0.419 \\
\hline Stroke $(\%)$ & $13(5)$ & $14(10)$ & $14(11)$ & 0.056 & 0.020 & 0.825 \\
\hline Cancer (\%) & $30(12)$ & $22(16)$ & $16(13)$ & 0.234 & 0.376 & 0.427 \\
\hline Anemia (\%) & $51(20)$ & $40(30)$ & $41(33)$ & 0.040 & 0.005 & 0.572 \\
\hline NYHA > II (\%) & $176(71)$ & $103(78)$ & $92(75)$ & 0.190 & 0.206 & 0.621 \\
\hline Rales (\%) & $77(31)$ & $64(48)$ & $68(55)$ & 0.003 & 0.000 & 0.247 \\
\hline Angina $(\%)$ & $44(17)$ & $30(22)$ & $33(27)$ & 0.231 & 0.062 & 0.425 \\
\hline NT-proBNP (ng/l), median [IQR] & 3546 [1816-6148] & $5046[2465-8297]$ & 5375 [2947-9238] & 0.000 & 0.000 & 0.881 \\
\hline $\operatorname{LVEF}(\%)$ & $29.3 \pm 7.5$ & $30.7 \pm 7.6$ & $29.4 \pm 8.2$ & 0.086 & 0.260 & 0.192 \\
\hline Body mass index $\left(\mathrm{kg} / \mathrm{m}^{2}\right)$ & $25.4 \pm 4.5$ & $25 \pm 3.9$ & $25.3 \pm 4.1$ & 0.292 & 0.363 & 0.554 \\
\hline Systolic blood pressure $(\mathrm{mmHg})$ & $119 \pm 18$ & $117 \pm 18$ & $116 \pm 17$ & 0.274 & 0.118 & 0.742 \\
\hline Hemoglobin $(\mathrm{g} / \mathrm{l})$ & $135 \pm 18$ & $129 \pm 20$ & $129 \pm 18$ & 0.005 & 0.001 & 0.996 \\
\hline Creatinine $(\mathrm{mmol} / \mathrm{l})$ & $104 \pm 32$ & $124 \pm 38$ & $133 \pm 41$ & 0.000 & 0.000 & 0.068 \\
\hline Potassium (mmol/l) & $4.0 \pm 0.4$ & $4.1 \pm 0.5$ & $4.2 \pm 0.5$ & 0.593 & 0.049 & 0.066 \\
\hline
\end{tabular}

Table 3.2: Baseline characteristics according to number of HF hospitalizations plus death.

${ }^{\dagger} \mathrm{P}_{01}$ : p-value comparison between those patients not hospitalized and those hospitalized only once (two sided t-test or Mann-Whitney U-test for continuous variables and $\chi^{2}$ test for categorical variables).

${ }^{\ddagger} \mathrm{P}_{0}$ : p-value comparison between those patients not hospitalized and those hospitalized at least once.

${ }^{\S} \mathrm{P}_{01}$ : p-value comparison between those patients hospitalized once and those hospitalized two times or more.

IQR: Interquartile range.

\subsubsection{Hazards of HF and all-cause Hospitalization}

The effect of NT-proBNP-guided therapy as compared to standard therapy on repeated hospitalizations/death, within 5.5 years follow-up, is presented in Table 3.3. Overall, the effect of NT-proBNP-guided therapy as compared to standard therapy on first all-cause hospitalization event (adjusted for baseline characteristics) was not statistically significant. However, there was a statistically significant beneficial effect of NT-proBNP-guided therapy on second and third all-cause hospitalization events. When considering prestratified age groups, these effects were only seen in patients aged between 60 and 74 years, but not in patients $\geq 75$ years (Table 3.3 ). 


\begin{tabular}{|c|c|c|c|c|c|c|}
\hline & Event & & p-value & $\mathrm{HR}(95 \% \mathrm{CI})$ & p-value & HR $(95 \%$ CI $)$ \\
\hline & & All patients & 010 & $084(069-103)$ & 035 & $091(075-111)$ \\
\hline & First & Age $<75$ & 0.04 & $0.71(0.51-0.98)$ & 0.06 & $0.73(0.53-1.0)$ \\
\hline & & Age $\geq 75$ & 0.87 & $0.97(0.75-1.26)$ & 0.83 & $1.02(0.79-1.33)$ \\
\hline & & All patients & 0.009 & $0.81(0.69-0.95)$ & 0.01 & $0.83(0.709-0.97)$ \\
\hline All-cause & Second & Age $<75$ & 0.004 & $0.68(0.53-0.88)$ & 0.01 & $0.70(0.54-0.92)$ \\
\hline hospitalization & & Age $\geq 75$ & 0.41 & $0.92(0.75-1.12)$ & 0.36 & $0.91(0.74-1.11)$ \\
\hline & & All patients & 0.03 & $0.85(0.74-0.98)$ & 0.04 & $0.87(0.754-0.99)$ \\
\hline & Third & Age $<75$ & 0.009 & $0.73(0.58-0.92)$ & 0.02 & $0.75(0.60-0.95)$ \\
\hline & & Age $\geq 75$ & 0.63 & $0.95(0.80-1.14)$ & 0.51 & $0.94(0.78-1.12)$ \\
\hline & & All patients & 0.006 & $0.70(0.55-0.90)$ & 0.01 & $0.73(0.575-0.939)$ \\
\hline & First & Age $<75$ & 0.004 & $0.51(0.33-0.80)$ & 0.006 & $0.53(0.34-0.83)$ \\
\hline $\mathrm{HF}$ & & Age $\geq 75$ & 0.26 & $0.84(0.62-1.13)$ & 0.23 & $0.83(0.62-1.12)$ \\
\hline & & All patients & 0.08 & $0.83(0.67-1.02)$ & 0.14 & $0.85(0.69-1.05)$ \\
\hline hospitalization & Second & Age $<75$ & 0.01 & $0.62(0.43-0.90)$ & 0.03 & $0.65(0.44-0.95)$ \\
\hline & & Age $\geq 75$ & 0.82 & $0.97(0.75-1.24)$ & 0.74 & $0.95(0.75-1.22)$ \\
\hline
\end{tabular}

Table 3.3: Estimations of Hazard ratios of NT-proBNP guided therapy compared to symptom-guided therapy effect on recurrence of events (adjusted for age, gender, coronary artery disease as main cause of $\mathrm{HF}$, Charlson Score, Kidney disease, ejection fraction centre, NYHA-class, and systolic blood pressure), using the PWP-GT model.

Overall, NT-proBNP-guided therapy showed a beneficial effect on first HF hospitalization event, again predominantly in the younger age group. For second HF hospitalization event, the beneficial effect of NT-proBNP-guided therapy was somewhat smaller in the older group and failed to reach statistical significance in analysis of the overall group. Again there was a difference between the younger and the older patient group.

\begin{tabular}{cccccc}
\hline & & $\begin{array}{c}\text { p-value } \\
\text { unadjusted }\end{array}$ & $\begin{array}{c}\text { Rate ratio (95\% CI) } \\
\text { unadjusted }\end{array}$ & $\begin{array}{c}\text { p-value } \\
\text { adjusted }\end{array}$ & $\begin{array}{c}\text { Rate ratio (95\% CI) } \\
\text { adjusted }\end{array}$ \\
\hline \multirow{3}{*}{ All-cause } & All patients & 0.49 & $0.94(0.79-1.11)$ & 0.46 & $0.93(0.79-1.11)$ \\
Age $<75$ & 0.32 & $0.86(0.63-1.16)$ & 0.43 & $0.89(0.67-1.19)$ \\
\hline HF & Age $\geq 75$ & 0.99 & $0.99(0.82-1.22)$ & 0.91 & $0.99(0.81-1.21)$ \\
\hline hospitalization & All patients & 0.15 & $0.84(0.68-10.6)$ & 0.12 & $0.84(0.67-10.04)$ \\
& Age $<75$ & 0.04 & $0.63(0.40-0.98)$ & 0.07 & $0.66(0.42-1.04)$ \\
& Age $\geq 75$ & 0.92 & $0.98(0.77-1.27)$ & 0.80 & $0.97(0.76-1.24)$ \\
\hline
\end{tabular}

Table 3.4: Estimations of Rate ratios of NT-proBNP guided therapy compared to symptom-guided therapy effect on recurrence of events (adjusted for age, gender, coronary artery disease as main cause of HF, Charlson Score, Kidney disease, ejection fraction centre, NYHA-class, and systolic blood pressure), using the Negative Binomial regression model. 
In this study our main focus was on treatment effect on recurrence time of events. For this, we used the PWP-GT model as explained. There are also methods to evaluate the association with numbers of all-cause and HF hospitalization events, such as the Negative Binomial regression model. These kinds of models do NOT take into account the timing of events, and therefore we feel this approach is less appropriate. However, we also modeled using the Negative Binomial regression model, (Table 3.4). In general, results are supporting our PWP-GT model results. Although the P-values are not significant, the rate ratios do go into the same direction of hazard ratios and show that NT-proBNPguided therapy reduces the rate of events (mainly for patients aged $<75$ ), however it failed to reach statistical significance.

\subsection{Discussion}

This chapter applied an approach for assessing treatment effects in HF patients on repeated events, which may be clinically more important for patients than first events only. We used the TIME-CHF data to investigate potential differences between the traditional approach and an adapted method that applies a Cox-regression not only for the first, but also for repeated events, i.e. the PWP-GT model. Interestingly, using NT-proBNP to guide intensification of HF medication to a greater extent than with standard care alone, resulted in reduction of repeated all-cause hospitalization events, whereas the time to the first event, i.e. all-cause hospitalization or death, was not significantly reduced. As repeated events may influence patient reported outcomes as well as costs more than first events only, the PWP-GT model may be preferable and even more powerful to reveal the effects of (new) interventions in diseases where repeated events are frequent and clinically relevant, such as in HF.

\subsubsection{Advantages of Considering Repeated Events}

To investigate the effectiveness of treatment in terms of hospitalization-free survival, standard approaches for survival analyses such as Cox regression models are usually applied, where repeated events are not taken into consideration. Obviously, this is the right approach if patients can suffer only one event (i.e. death) and other events are not considered, repeated events are scarce, or if effects are similar on composite endpoints and do not differ on consecutive endpoints [8]. However, taking only the first event into account might underestimate the effect of treatment in complex chronic diseases such as HF. This is in line with our findings that effects on the primary endpoint all-cause hospitalization free survival was only revealed when considering repeated events, whereas first events were primarily reduced for the disease-specific HF related endpoint. Consecutive HFrelated events were somewhat less affected. Therefore, in order to have a more accurate 
estimation, it makes more sense to take recurring events and their corresponding time intervals into consideration [28, 106], particularly for all-cause events in complex diseases. Additional similar analyses of trials in complex diseases may help to get more insight in possible differences in the response of repeated events, depending on the selected endpoint and their potential clinical consequences.

When analyzing the times between the recurrences of events, these times, which form the outcomes, may vary depending on different number of events per individual patient. Thus, the outcomes are likely to be correlated because they belong to an individual patient [41]. The PWP-GT model is not only able to evaluate the treatment effect on the time until the first event, but also can be used to assess its effect on consecutive events [54, 106]. This approach considers the time from baseline to the first hospitalization event, time from onset of the first until the second hospitalization event, and onset of the second until the third hospitalization event, and so forth, as the outcome variables of interest. Each patient contributes a set of data for each time interval (gap-time) corresponding to each repeated event. Patients are not restricted to have the same number of outcomes, data sets, and follow-up times and data sets of patients in their $k^{\text {th }}$ event construct the $k^{\text {th }}$ stratum.

The PWP-GT model, models the hazard of a subsequent event conditionally on the entire sequence of previous events experienced by an individual patient, in each stratum $[28,106]$. In other words, this model specifies how the hazard of subsequent recurrences depends on the past event history, in contrast to the classical Cox regression model which models the hazard of an event regardless of the past event history. In the PWP-GT model, we apply robust sandwich variance estimators [74, 147], to adjust the variances of the estimated regression coefficients for the correlation of observations in the same patient.

Although there are a number of approaches available for analyzing repeated events $[28,55]$, the PWP-GT model is recommended in the case that there is a strong relationship between the first and subsequent events, and if one is more interested in the separate risk for these events [45]. Unlike other approaches for analyzing repeated events, the PWP-GT model takes into account the time between repeated events and intra-individual correlation of events within a patient, making it particularly suitable for investigating repeated events in chronic diseases.

\subsubsection{Potential Impact of Considering Events - TIME-CHF as Example}

TIME-CHF was a neutral study as reduction of the primary endpoint by the NT-proBNPguided versus symptom-guided therapy, i.e. all-cause hospitalization event, was not achieved. Interestingly, considering repeated events of the primary endpoint instead of time-to-first events, NT-proBNP-guided therapy resulted in a significant benefit as compared to symptom guided therapy, which is in line with the positive findings on the disease-specific endpoint [102], also found in this analysis, the positive effects on left-ventricular ejec- 
tion fraction [61], the excellent safety profile [119], and the maintained long-term effects, at least in younger patients [118]. Despite these findings, TIME-CHF obviously cannot be considered as a positive trial on the primary endpoint and this analysis is hypothesis generating only regarding the clinical consequences. It might be seen as reassuring that repeated all-cause hospitalization events are not negatively influenced by intensifying HF medication even in the very elderly patients, supporting the positive safety profile of such an intervention.

\subsubsection{Limitations}

This study has some limitations that need to be addressed. First, the PWP-GT model, though very promising and addressing a relevant question in chronic diseases, is a relatively new method that needs to be further tested before it can be widely recommended. Second, this study differentially analyzed the primary and a secondary endpoint of a trial that was not defined per protocol and must be considered as post-hoc analysis only, not proving superiority of NT-proBNP-guided therapy on the primary endpoint in TIMECHF. Moreover, GUIDE-IT (Guiding evidence based therapy using biomarker Intensified Treatment in heart failure) seems to be neutral, but as no further information is available, published data must be awaited for further interpretation, which is expected in 2018 [1]. It is, however, important to note that the aim of this analysis is primarily to investigate potential differences between methods to test the effects of interventions on outcome and not on the role of NT-proBNP-guided therapy. Third, the method was not able to differentiate fourth and consecutive events due to lack of a sufficient number of such events. Fourth, the method is not able to distinguish death from hospitalization as event. Theoretically, the effects of an intervention on mortality might influence results on repeated events (e.g. longer survival may increase likelihood of repeated hospitalization). Still, we think that this is unlikely in this study, as effects on mortality overall and in the two age groups are very much in line with the results of this analysis (effects of NT-proBNP-guided therapy as compared symptom-guided therapy on mortality: overall $\mathrm{HR}=0.85, \mathrm{p}=0.25$; age $60-74$ years: $H R=0.62, p=0.06$; age $\geq 75$ years: $H R=1.07, p=0.71$ ) [19]. Moreover, considering only hospitalizations as events and censoring death did not influence results significantly (data not shown). Fifth, there is no method available for calculating the PWP-GT model's power for the whole model as well as for consecutive events. In the present analysis, statistical power may be particularly low for all-cause hospitalization or death as third event and interpretation of it must be done with great caution. In addition, statistical methods need to be developed to test power of the PWP-GT model, which is also true for other analyses considering repeated events. 
36 Chapter 3. NT-proBNP-Guided Therapy Reduces Repeated Heart Failure Hospitalizations

\subsection{Conclusions}

Our analysis shows that NT-proBNP-guided therapy reduces the risk of recurrent events in patients aged $<75$ years. This included all-cause hospitalization by mainly reducing later events, adding knowledge to the neutral effect on this endpoint when shown using time-to-first event analysis only. Our findings highlight the relevance of new methods to address the clinically important question of the effects of interventions on repeated events. The PWP-GT model may be promising, but needs to be further evaluated before widely used for assessing the primary endpoint in future intervention studies in chronic diseases such as HF, where repeated events are a clinically important problem. 


\section{Cutpoint Estimation for Continuous Covariates Dichotomization}

This chapter is based on: Davarzani, N., Achcar, J.A., Karel, J. Smirnov, E., Brunner-La Rocca, H.P., Peeters, R. Cutpoint Estimation for Continuous Covariates Dichotomization. Submitted. 


\begin{abstract}
Dichotomizing continuous covariates is widespread in medical prognostic variable studies. Dichotomization of a covariate makes it easier for clinicians to interpret the covariate's effect on the outcome variable and guides them in their choice of therapy. The dichotomization point (cutpoint) for a covariate can be selected or estimated based on the data distribution or in terms of the covariate's effect on an outcome variable. In this chapter we introduce a Bayesian approach to estimate cutpoints for continuous covariates when dichotomized covariates are related to an outcome variable through linear regression. Using Markov Chain Monte Carlo (MCMC) methods, we obtain Bayesian estimates for the cutpoints and their corresponding credible intervals. The proposed Bayesian approach does not limit the selected cutpoint to the observed values of the covariate and the cutpoint can be any value in the range of the covariate. The proposed approach can be used to dichotomize a subset or all the covariates when we are using a multivariate regression model. To the best of our knowledge this is the first method which can deal with multivariate dichotomization with respect to modeling a continuous outcome variable. We validate the proposed Bayesian approach on data from a simulation study and we demonstrate the use of this approach on the TIME-CHF study.
\end{abstract}




\subsection{Introduction}

In medical studies, regression models are often used to investigate the relationship between covariates and an outcome variable. Covariates can be dosages of medications, patient characteristics, the levels of biomarkers, etc., and are usually measured on a continuous scale. In practice, however, clinicians may prefer to use them in a dichotomized form in their analysis. This arises from the fact that using covariates in a dichotomized form makes it easier for clinicians to interpret the relationship between an outcome variable and covariates and helps them to make treatment decisions.

From a statistical point of view, dichotomized covariates may also be preferred for several other reasons: First, the interpretation of the effects of the covariates on the outcome variable may be easier. For example, effect measures from statistical models such as hazard ratio or odds ratio are easier to interpret for binary covariates, especially in multivariate models. Moreover, using the covariates in dichotomized form will be helpful for interpreting the interaction effect of more than two covariates in a model. Second, it makes data summarization more efficient. Third, sometimes it is very difficult to have exact measurements of a continuous variable, therefore by dichotomizing the variable one may be less concerned about measurement errors. However, dichotomizing a continuous variable may also lead to loss of statistical power [26, 92] and lead to a higher type-I error $[12,108]$.

In the literature, there are two common types of methods for choosing the cutpoints for dichotomizing continuous covariates: exploratory methods and outcome-oriented methods [82]. In exploratory methods, based on a data distribution, some value or percentile of the data can be proposed as a cutpoint to dichotomize a continuous variable. In outcomeoriented methods, the cutpoint is defined as a value of the continuous covariate that best separates the data into two groups with respect to the outcome, using statistical criteria. In many studies, when the objective is assessing the relationship between a covariate and an outcome variable, one may explore the cutpoint through a regression model. Cutpoint selection with the use of regression models has been proposed for different types of data (with discrete or continuous outcomes, lifetime outcomes and longitudinal outcomes), by a number of authors (see for example [33, 92, 149]).

Our main focus in this chapter is on outcome-oriented methods. Most of the methods of this type, proposed so far in the literature, consider a selection of observed values of the covariate and choose one of those as the cutpoint based on statistical criteria [92, 134]. For instance, they choose the value which best separates the classes according to the minimum Chi-squared p-value. Unfortunately, as a drawback this method is well known to possibly inflate the type-I error, due to multiple testing [9].

Breiman et al. [18] introduced Classification and Regression Trees (CART) as a non- 
parametric method to build a decision tree. The CART method essentially selects that value as a cutpoint that splits the data into two most homogeneous groups based on the Gini index.

O'Brien [92] proposed a method based on the assumption that the outcome variable $Y$ and covariate $X$ are related through a conditional distribution $f(Y \mid X)$ which preferably belongs to an exponential family of distributions. Then based on each covariate value (cutpoint candidate) subjects will be split up into two groups. The best cutpoint is defined as the candidate that minimizes a measure of distance between the true expected value of the outcome for each subject and the estimated average outcome among subjects in the same group.

Tunes-da-Silva and Klein [134] proposed a test statistic to select a cutpoint for a continuous covariate when performing a regression analysis based on generalized estimating equations.

Liu [75] defined a concordance probability as a criterion evaluating the classification accuracy of a dichotomized covariate, which is defined as a function of sensitivity and specificity at the cutpoint. He applied a nonparametric approach to search for the cutpoint, maximizing the concordance probability.

To the best of our knowledge all outcome-oriented methods (with respect to a continuous outcome) in the literature derive the cutpoint using univariate analysis for a single covariate only, and there is no method dealing directly with multivariate dichotomization. Moreover, in most of them the choice of cutpoint is limited to the observed values of the covariate.

In this chapter, we propose employing a Bayesian approach for dichotomizing continuous covariates when assuming the dichotomized covariate is related to the outcome variable through a regression model. The method can be used both in univariate and in mutivariate settings. We assume there exists prior knowledge of the regression parameters and that the cutpoint may be characterized by prior distributions. Using new data, we can update the prior knowledge and obtain the corresponding posterior distributions of the unknown parameters. Then, by deriving the posterior distribution of the cutpoint we can obtain the Bayes estimate of the cutpoint for the continuous covariate with respect to any feasible loss function. However, we often see that the posterior distributions of the parameters do not exist in a closed form and therefore we use Markov Chain Monte Carlo (MCMC) methods such as the Gibbs sampling algorithm [38] or the Metropolis-Hastings (M-H) algorithm [23], to estimate the parameters (see Section 2.3). In this case, in addition to finding the Bayesian point estimates of the parameters (the cutpoint and regression parameters) we can also compute credible intervals.

The proposed Bayesian approach does not limit the selected cutpoint to the observed values of the covariate anymore and it can be applied in a multivariate setting. In addition, by employing the Bayesian approach, the inflation of the type-I error is no longer an issue 
due to avoiding multiple testing. In most of the classical approaches, due to lack of a pivotal quantity [52], we are not able to find a confidence interval for the determined cutpoint. However, with the use of the Bayesian approach, it is straightforward to derive a credible interval for the estimated cutpoint.

The rest of the chapter is organized as follows: In Section 4.2, by setting up a multivariate regression model we apply a Bayesian approach to estimate the cutpoints for dichotomizing continuous covariates. In Section 4.3, we conduct a simulation study to assess the properties of the proposed Bayesian approach employing non-informative priors. In Section 4.4, we employ the proposed Bayesian approach, to dichotomize some covariates in the TIME-CHF study. Finally in Section 4.5 we conclude this chapter by discussing the obtained results, disadvantages of the proposed approach and future work.

\subsection{Dichotomization Using Linear Regression Models}

In this section, we consider multivariate dichotomization of continuous covariates. We assume that covariates in dichotomized form are related to the outcome variable through a multivariate linear regression model. In this set up we employ a Bayesian approach to estimate the cutpoints for the covariates. Since we are not able to find the Bayes estimates of the regression parameters and cutpoints in a closed form we propose using MCMC methods to approximate these values.

Suppose that $Y_{i} \in \mathbb{R}, i=1, \ldots, n$, are independent continuous outcome variables for $n$ subjects. Assume for each subject there exist $p$ continuous covariates $X_{1}, \ldots, X_{p}$. Let $\boldsymbol{X}_{l}=\left(X_{1 l}, \ldots, X_{n l}\right)^{\prime}$ denote the vector of values for the $l^{\text {th }}$ covariate, $l=1, \ldots, p$, for all subjects and $\boldsymbol{X}=\left(\boldsymbol{X}_{1}, \boldsymbol{X}_{2}, \ldots, \boldsymbol{X}_{p}\right)$ be the matrix of covariate values. Let $T_{1 l}=$ $\min \left(\boldsymbol{X}_{l}\right)$ and $T_{2 l}=\max \left(\boldsymbol{X}_{l}\right)$, then for the $i^{\text {th }}$ subject define $Z_{i l}$ as the dichotomized covariate obtained from $\boldsymbol{X}_{l}$ at cutpoint $\delta_{l}, i=1, \ldots, n, l=1, \ldots, p$, i.e.,

$$
Z_{i l}=\left\{\begin{array}{lll}
0 & \text { if } & X_{i l} \leq \delta_{l} \\
1 & \text { if } \quad & X_{i l}>\delta_{l},
\end{array}\right.
$$

where, $\delta_{l} \in\left(T_{1 l}, T_{2 l}\right)$. To estimate the cutpoint $\delta_{l}$, we assume that the outcome variable and the dichotomized covariates are related through the following multivariate regression model:

$$
Y_{i}=\boldsymbol{Z}_{i}^{\prime} \boldsymbol{\beta}+\epsilon_{i}, i=1, \ldots, n,
$$

where, $\epsilon_{i} \sim \mathcal{N}\left(0, \sigma^{2}\right), \boldsymbol{Z}_{i}^{\prime}=\left(1, Z_{i 1}, \ldots, Z_{i p}\right)$ and $\boldsymbol{\beta}^{\prime}=\left(\beta_{0}, \beta_{1}, \ldots, \beta_{p}\right)$. Then the joint likelihood function of $\boldsymbol{\beta}, \boldsymbol{\delta}=\left(\delta_{1}, \ldots, \delta_{p}\right)$ and $\sigma^{2}$ given realizations $\boldsymbol{y}=\left(y_{1}, \ldots, y_{n}\right)^{\prime}$ and $\boldsymbol{x}=\left(x_{1}, \ldots, x_{n}\right)^{\prime}$ is 


$$
L\left(\boldsymbol{\beta}, \sigma^{2}, \boldsymbol{\delta} \mid \boldsymbol{y}, \boldsymbol{x}\right) \propto\left(\sigma^{2}\right)^{-n / 2} \exp \left(-\frac{1}{2 \sigma^{2}}(\boldsymbol{y}-\boldsymbol{z} \boldsymbol{\beta})^{\prime}(\boldsymbol{y}-\boldsymbol{z} \boldsymbol{\beta})\right) .
$$

where, $\boldsymbol{z}=\left(\boldsymbol{z}_{1}, \ldots, \boldsymbol{z}_{n}\right)^{\prime}$ is an $n \times(p+1)$ matrix which depends on $\boldsymbol{x}$ and $\boldsymbol{\delta}$.

\subsubsection{Bayesian Parameter Estimation}

Specifying an appropriate prior distribution for the parameter is a key aspect in a Bayesian analysis. The prior distribution for a parameter is called a conjugate prior [109] if it is chosen such that the posterior distribution follows the same family as the prior distribution. By choosing a conjugate prior we will have a known class for the posterior distribution which is a computational convenience.

Let us assume the joint conjugate prior (see, $[42,140])$ for $\left(\boldsymbol{\beta}, \sigma^{2}\right)$ to be independent of the joint prior distribution for the cutpoints, and to have the following structure:

$$
\pi\left(\boldsymbol{\beta}, \sigma^{2}\right)=\pi\left(\boldsymbol{\beta} \mid \sigma^{2}\right) \pi\left(\sigma^{2}\right)
$$

where the conditional prior for $\boldsymbol{\beta}$ given $\sigma^{2}$ is the multivariate normal distribution, $\mathcal{N}\left(\boldsymbol{\mu}_{0}, \sigma^{2} \mathbf{V}_{0}^{-1}\right)$ with a known mean $\boldsymbol{\mu}_{0}$ and covariance matrix $\sigma^{2} \mathbf{V}_{0}^{-1}$, where $\mathbf{V}_{0}$ is a known matrix. Assume that the prior for $\sigma^{2}$ is the Inverse Gamma distribution, $\operatorname{IG}\left(a_{0}, b_{0}\right)$, with hyperparameters $a_{0}$ and $b_{0}$. Let us assume that $\delta_{l}, l=1, \ldots, p$, have independent prior distributions $\pi_{l}\left(\delta_{l}\right)$, where $\delta_{l} \in\left(T_{1 l}, T_{2 l}\right)$. Then the joint posterior density function of $\boldsymbol{\beta}, \sigma^{2}$ and $\boldsymbol{\delta}$ is:

$$
\begin{aligned}
\pi\left(\boldsymbol{\beta}, \sigma^{2}, \boldsymbol{\delta} \mid \boldsymbol{y}, \boldsymbol{x}\right) & \propto\left(\sigma^{2}\right)^{-n / 2} \exp \left(-\frac{1}{2 \sigma^{2}}(\boldsymbol{y}-\boldsymbol{z} \boldsymbol{\beta})^{\prime}(\boldsymbol{y}-\boldsymbol{z} \boldsymbol{\beta})\right) \\
& \times\left(\sigma^{2}\right)^{-p / 2} \exp \left(-\frac{1}{2 \sigma^{2}}\left(\boldsymbol{\beta}-\boldsymbol{\mu}_{0}\right)^{\prime} \boldsymbol{V}_{0}\left(\boldsymbol{\beta}-\boldsymbol{\mu}_{0}\right)\right) \\
& \times\left(\sigma^{2}\right)^{-\left(a_{0}+1\right)} \exp \left(-\frac{b_{0}}{\sigma^{2}}\right) \prod_{l=1}^{p} \pi_{l}\left(\delta_{l}\right) .
\end{aligned}
$$

Let $\boldsymbol{\mu}=\left(\boldsymbol{z}^{\prime} \boldsymbol{z}+\boldsymbol{V}_{0}\right)^{-1}\left(\boldsymbol{z}^{\prime} \boldsymbol{y}+\boldsymbol{V}_{0} \boldsymbol{\mu}_{0}\right)$. Then the quadratic terms in (4.5) can be rewritten as:

$$
\begin{aligned}
& (\boldsymbol{y}-\boldsymbol{z} \boldsymbol{\beta})^{\prime}(\boldsymbol{y}-\boldsymbol{z} \boldsymbol{\beta})+\left(\boldsymbol{\beta}-\boldsymbol{\mu}_{0}\right)^{\prime} \boldsymbol{V}_{0}\left(\boldsymbol{\beta}-\boldsymbol{\mu}_{0}\right)= \\
& (\boldsymbol{\beta}-\boldsymbol{\mu})^{\prime}\left(\boldsymbol{z}^{\prime} \boldsymbol{z}+\boldsymbol{V}_{0}\right)(\boldsymbol{\beta}-\boldsymbol{\mu})+\boldsymbol{y}^{\prime} \boldsymbol{y}-\boldsymbol{\mu}^{\prime}\left(\boldsymbol{z}^{\prime} \boldsymbol{z}+\boldsymbol{V}_{0}\right) \boldsymbol{\mu}+\boldsymbol{\mu}_{0}^{\prime} \boldsymbol{V}_{0} \boldsymbol{\mu}_{0}
\end{aligned}
$$

From (4.5) and (4.6), the conditional posterior density function of $\boldsymbol{\beta}$ given $\sigma^{2}$ and $\boldsymbol{\delta}$ is

$$
\pi\left(\boldsymbol{\beta} \mid \sigma^{2}, \boldsymbol{\delta}, \boldsymbol{y}, \boldsymbol{x}\right) \propto \exp \left(-\frac{1}{2 \sigma^{2}}(\boldsymbol{\beta}-\boldsymbol{\mu})^{\prime} \boldsymbol{V}(\boldsymbol{\beta}-\boldsymbol{\mu})\right)
$$


and thus,

$$
\boldsymbol{\beta} \mid \sigma^{2}, \boldsymbol{\delta}, \boldsymbol{y}, \boldsymbol{x} \sim \mathcal{N}\left(\boldsymbol{\mu}, \sigma^{2} \boldsymbol{V}^{-1}\right),
$$

where, $\boldsymbol{V}=\boldsymbol{z}^{\prime} \boldsymbol{z}+\boldsymbol{V}_{0}$. The conditional posterior density function of $\sigma^{2}$ given $\boldsymbol{\delta}$ is:

$$
\pi\left(\sigma^{2} \mid \boldsymbol{\delta}, \boldsymbol{y}, \boldsymbol{x}\right) \propto\left(\sigma^{2}\right)^{-\left(n+2 a_{0}\right) / 2-1} \exp \left(-\frac{2 b_{0}+\boldsymbol{y}^{\prime} \boldsymbol{y}-\boldsymbol{\mu}^{\prime} \boldsymbol{V} \boldsymbol{\mu}+\boldsymbol{\mu}_{0}^{\prime} \boldsymbol{V}_{0} \boldsymbol{\mu}_{0}}{2 \sigma^{2}}\right)
$$

and thus,

$$
\sigma^{2} \mid \boldsymbol{\delta}, \boldsymbol{y}, \boldsymbol{x} \sim \operatorname{IG}(a, b)
$$

where $a=a_{0}+n / 2$ and $b=b_{0}+\frac{1}{2}\left(\boldsymbol{y}^{\prime} \boldsymbol{y}-\boldsymbol{\mu}^{\prime} \boldsymbol{V} \boldsymbol{\mu}+\boldsymbol{\mu}_{0}^{\prime} \boldsymbol{V}_{0} \boldsymbol{\mu}_{0}\right)$.

Finally, the conditional posterior density function of $\boldsymbol{\delta}$ given $\boldsymbol{\beta}$ and $\sigma^{2}$, is:

$$
\pi\left(\boldsymbol{\delta} \mid \boldsymbol{\beta}, \sigma^{2}, \boldsymbol{y}, \boldsymbol{x}\right) \propto \exp \left(-\frac{1}{2 \sigma^{2}}(\boldsymbol{y}-\boldsymbol{z} \boldsymbol{\beta})^{\prime}(\boldsymbol{y}-\boldsymbol{z} \boldsymbol{\beta})\right) \prod_{l=1}^{p} \pi_{l}\left(\delta_{l}\right) .
$$

From the conditional posterior distributions (4.7) - (4.9), we can generate samples for $\boldsymbol{\beta}, \boldsymbol{\delta}$ and $\sigma^{2}$ using the Gibbs sampling algorithm [38] to compute their corresponding Bayes estimates (see Section 2.3). Note that since the conditional posterior density function for $\delta$ has no closed form, we use the M-H algorithm to generate samples from $\pi\left(\boldsymbol{\delta} \mid \boldsymbol{\beta}, \sigma^{2}, \boldsymbol{y}, \boldsymbol{x}\right)$ (see Section 2.3). Once we have the MCMC samples, it is possible to compute the Bayes estimates of any function of the unknown parameters with respect to any loss function.

Alternatively we can assume non-informative prior distributions for the parameters. This will make the estimated cutpoints less dependent on the prior information and more comparable to cutpoints obtained by classical methods. A non-informative prior distribution that is commonly used for $\boldsymbol{\beta}$ and $\sigma^{2}$ in a multivariate linear regression [42] is:

$$
\pi\left(\boldsymbol{\beta}, \sigma^{2}\right) \propto \frac{1}{\sigma^{2}}
$$

which can be obtained from the conjugate prior (4.4) with $a_{0}=-p / 2, b_{0}=0$ and $\boldsymbol{V}_{0} \rightarrow \mathbf{0}$ [94].

Assume also that $\delta_{l}, l=1, \ldots, p$, have independent uniform prior distributions that are $\mathrm{U}\left(T_{l 1}, T_{l 2}\right)$. Then the joint posterior density function of $\boldsymbol{\beta}, \sigma^{2}$ and $\boldsymbol{\delta}$ is:

$$
\pi\left(\boldsymbol{\beta}, \sigma^{2}, \boldsymbol{\delta} \mid \boldsymbol{y}, \boldsymbol{x}\right) \propto\left(\sigma^{2}\right)^{-(n+2) / 2} \exp \left(-\frac{1}{2 \sigma^{2}}(\boldsymbol{y}-\boldsymbol{z} \boldsymbol{\beta})^{\prime}(\boldsymbol{y}-\boldsymbol{z} \boldsymbol{\beta})\right)
$$


Furthermore, the conditional posterior density function of $\boldsymbol{\beta}$ given $\sigma^{2}$ and $\boldsymbol{\delta}$ is

$$
\pi\left(\boldsymbol{\beta} \mid \sigma^{2}, \boldsymbol{\delta}, \boldsymbol{y}, \boldsymbol{x}\right) \propto \exp \left(-\frac{1}{2 \sigma^{2}}\left(\boldsymbol{\beta}-\boldsymbol{\mu}_{1}\right)^{\prime} \boldsymbol{V}_{1}\left(\boldsymbol{\beta}-\boldsymbol{\mu}_{1}\right)\right),
$$

that is,

$$
\boldsymbol{\beta} \mid \sigma^{2}, \boldsymbol{\delta}, \boldsymbol{y}, \boldsymbol{x} \sim \mathcal{N}\left(\boldsymbol{\mu}_{1}, \sigma^{2} \boldsymbol{V}_{1}^{-1}\right),
$$

where $\boldsymbol{V}_{1}=\boldsymbol{z}^{\prime} \boldsymbol{z}$ and $\boldsymbol{\mu}_{1}=\boldsymbol{V}_{1}^{-1} \boldsymbol{z}^{\prime} \boldsymbol{y}$. The conditional posterior density function of $\sigma^{2}$ given $\delta$ is

$$
\pi\left(\sigma^{2} \mid \boldsymbol{\delta}, \boldsymbol{y}, \boldsymbol{x}\right) \propto\left(\sigma^{2}\right)^{-(n-p) / 2-1} \exp \left(-\frac{\boldsymbol{y}^{\prime} \boldsymbol{y}-\boldsymbol{\mu}_{1}^{\prime} \boldsymbol{V}_{1} \boldsymbol{\mu}_{1}}{2 \sigma^{2}}\right)
$$

and thus,

$$
\sigma^{2} \mid \boldsymbol{\delta}, \boldsymbol{y}, \boldsymbol{x} \sim \mathrm{IG}\left(a_{1}, b_{1}\right),
$$

where $a_{1}=(n-p) / 2$ and $b_{1}=\frac{1}{2}\left(\boldsymbol{y}^{\prime} \boldsymbol{y}-\boldsymbol{\mu}_{1}^{\prime} \boldsymbol{V}_{1} \boldsymbol{\mu}_{1}\right)$.

The conditional posterior density function of $\boldsymbol{\delta}$ given $\boldsymbol{\beta}$ and $\sigma^{2}$, is

$$
\pi\left(\boldsymbol{\delta} \mid \boldsymbol{\beta}, \sigma^{2}, \boldsymbol{y}, \boldsymbol{x}\right) \propto \exp \left(-\frac{1}{2 \sigma^{2}}(\boldsymbol{y}-\boldsymbol{z} \boldsymbol{\beta})^{\prime}(\boldsymbol{y}-\boldsymbol{z} \boldsymbol{\beta})\right) .
$$

Note that in the proposed multivariate setting we can either dichotomize all the covariates in the model (in case all of them are continuous) or only dichotomize a subset of covariates. For example, suppose we are interested in dichotomizing covariates $X_{1}, \ldots, X_{p-1}$, in the presence of $X_{p}$. Define $Z_{i}^{\prime}=\left(1, Z_{i 1}, \ldots, Z_{i(p-1)}, X_{i p}\right), i=$ $1, \ldots, n$ in the multivariate regression model (4.2) and $\boldsymbol{\delta}=\left(\delta_{1}, \ldots, \delta_{p-1}\right)$. Assume the joint prior distribution $\prod_{l=1}^{p-1} \pi_{l}\left(\delta_{l}\right)$ for $\boldsymbol{\delta}$ and joint priors (4.4) or (4.10) for $\boldsymbol{\beta}$ and $\sigma^{2}$. Then we can follow the same procedure as above to estimate the cutpoints and the regression parameters.

\subsection{Simulation study}

In this section, we perform an analysis using simulated data to assess the performance of the proposed Bayesian approach in estimating cutpoints of covariates in univariate and bivariate settings. In the univariate setting we assume there is a continuous covariate $X_{1}$ such that its corresponding dichotomized covariate $Z_{1}$, at true cutpoint $\delta_{1}$, is related to the outcome variable $Y$ through the univariate regression model $Y=\beta_{0}+\beta_{1} Z_{1}+\epsilon$, 
where $\epsilon \sim \mathcal{N}\left(0, \sigma^{2}\right)$. Likewise in the bivariate setting we assume there are covariates $X_{1}$ and $X_{2}$ with dichotomized covariates $Z_{1}$ and $Z_{2}$, at true cutpoints $\delta_{1}$ and $\delta_{2}$, respectively, which are related to the outcome variable through the bivariate regression $Y=\beta_{0}+$ $\beta_{1} Z_{1}+\beta_{2} Z_{2}+\epsilon$.

In the simulation study we evaluate the performance of the Bayesian approach in estimating the true cutpoints points and the regression parameters. To this end we consider data sets with different numbers of subjects to observe the effect of the size of the data on our estimations. Letting the data be scaled such that the covariates range between 0 and 50 , we then consider situations for which the true cutpoints range from the median to the $90^{\text {th }}$ percentile of the covariates. In addition we assess the effect of the variance of the error term, when it ranges from 0.01 to 9 , on the performance of the Bayesian approach. We perform the simulation study in a univariate and in a bivariate setting in the following scenarios:

\section{In the univariate setting:}

1. $X_{1} \in[0,50], \beta_{0}=1, \beta_{1}=-2, \delta_{1}=25, \sigma^{2}=1$ and $n=50,100,200,500$.

2. $X_{1} \in[0,50], \beta_{0}=1, \beta_{1}=-2, \delta_{1}=25,30,35,40,45, \sigma^{2}=1$ and $n=500$.

3. $X_{1} \in[0,50], \beta_{0}=1, \beta_{1}=-2, \delta_{1}=25, \sigma^{2}=0.01,0.25,1,4,9$ and $n=500$.

\section{In the bivariate setting:}

1. $X_{1} \in[0,50], X_{2} \in[0,50], \beta_{0}=1, \beta_{1}=-2, \beta_{2}=2, \delta_{1}=25, \delta_{2}=25, \sigma^{2}=1$ and $n=50,100,200,500,1000$.

2. $X_{1} \in[0,50], X_{2} \in[0,50], \beta_{0}=1, \beta_{1}=-2, \beta_{2}=2,\left(\delta_{1}, \delta_{2}\right)=(25,25)$, $(30,30),(35,35),(40,40),(45,45), \sigma^{2}=1$ and $n=500$.

3. $X_{1} \in[0,50], X_{2} \in[0,50], \beta_{0}=1, \beta_{1}=-2, \beta_{2}=2, \delta_{1}=25, \delta_{2}=25$, $\sigma^{2}=0.01,0.25,1,4,9$ and $n=500$.

In each scenario, for the given parameters, we generate $k=1000$ data sets. We assume the non-informative prior in (4.10) for the regression parameters and uniform $(0,50)$ prior distributions for the cutpoints $\delta_{1}$ and $\delta_{2}$. Then for a given data set we apply the Gibbs sampling algorithm to generate 10000 samples from the conditional posterior density functions (4.12)-(4.14), with burn-in period $=1000$. We note that within the Gibbs sampling algorithm, in order to sample $\delta_{1}$ and $\delta_{2}$ from their posterior density functions (4.14), we use the M-H algorithm. Then for the $i^{\text {th }}$ data set, we obtain the Bayes estimates for the cutpoints $\hat{\delta}_{1 i}, \hat{\delta}_{2 i}$ and for the regression coefficients $\left(\hat{\beta}_{0 i}, \hat{\beta}_{1 i}, \hat{\beta}_{2 i}\right), i=1, \ldots, k$, under the Squared Error Loss (SEL) function. 


\begin{tabular}{cccccc}
\hline$\delta=25, \sigma^{2}=1$ & \multicolumn{5}{c}{ Parameter quality (MSE and bias) } \\
\hline $\mathrm{n}$ & $\delta$ (O’Brien) & $\delta$ (Bayes) & $\beta_{0}$ & $\beta_{1}$ & $\sigma^{2}$ \\
\hline \multirow{2}{*}{50} & 4.36784 & 2.55616 & 0.04225 & 0.07991 & 0.05813 \\
& $(-0.84824)$ & $(-0.00085)$ & $(-0.0068)$ & $(0.01385)$ & $(0.07337)$ \\
\hline \multirow{2}{*}{100} & 1.05984 & 0.60995 & 0.02035 & 0.03951 & 0.02371 \\
& $(-0.42401)$ & $(-0.0339)$ & $(0.00067)$ & $(0.00807)$ & $(0.02354)$ \\
\hline \multirow{2}{*}{200} & 0.28496 & 0.147 & 0.01012 & 0.01919 & 0.01038 \\
& $(-0.18848)$ & $(0.014)$ & $(0.00016)$ & $(0.00437)$ & $(0.00763)$ \\
\hline \multirow{2}{*}{500} & 0.04349 & 0.02236 & 0.00382 & 0.00773 & 0.0039 \\
& $(-0.08043)$ & $(0.00178)$ & $(-0.00014)$ & $(0.00174)$ & $(0.00499)$ \\
\hline
\end{tabular}

Table 4.1: Quality of cutpoints and regression parameters using a univariate linear regression model, when $X_{1} \in[0,50], \beta_{0}=1, \beta_{1}=-2, \delta_{1}=25, \sigma^{2}=1$ and $n=50,100$, 200,500

In each box the first row presents the $\widehat{\mathrm{MSE}}$ of the estimates and in the second row the corresponding bias is reported within parentheses.

\begin{tabular}{cccccc}
\hline$n=500, \sigma^{2}=1$ & \multicolumn{5}{c}{ Parameter quality (MSE and bias) } \\
\hline$\delta$ & $\delta$ (O’Brien) & $\delta$ (Bayes) & $\beta_{0}$ & $\beta_{1}$ & $\sigma^{2}$ \\
\hline \multirow{2}{*}{25} & 0.04349 & 0.02236 & 0.00382 & 0.00773 & 0.0039 \\
& $(-0.08043)$ & $(0.00178)$ & $(-0.00014)$ & $(0.00174)$ & $(0.00499)$ \\
\hline \multirow{2}{*}{30} & 0.04982 & 0.02535 & 0.00353 & 0.00875 & 0.00413 \\
& $(-0.07994)$ & $(-0.00222)$ & $(-0.00251)$ & $(0.00722)$ & $(0.00739)$ \\
\hline \multirow{2}{*}{35} & 0.05347 & 0.02615 & 0.00307 & 0.00978 & 0.00409 \\
& $(-0.09206)$ & $(-0.00259)$ & $(-0.00137)$ & $(0.00251)$ & $(0.00323)$ \\
\hline \multirow{2}{*}{40} & 0.04037 & 0.02424 & 0.00275 & 0.01303 & 0.00401 \\
& $(-0.07695)$ & $(0.00633)$ & $(-0.00119)$ & $(-0.00295)$ & $(0.00748)$ \\
\hline \multirow{2}{*}{45} & 0.04164 & 0.02398 & 0.00223 & 0.02168 & 0.00433 \\
& $(-0.08028)$ & $(-0.00612)$ & $(-0.00389)$ & $(0.01634)$ & $(0.00639)$ \\
\hline
\end{tabular}

Table 4.2: Quality of cutpoints and regression parameters using a univariate linear regression model, when $X_{1} \in[0,50], \beta_{0}=1, \beta_{1}=-2, \delta_{1}=25,30,35,40,45, \sigma^{2}=1$ and $n=500$.

In each box the first row presents the $\widehat{\mathrm{MSE}}$ of the estimates and in the second row the corresponding bias is reported within parentheses. 


\begin{tabular}{cccccc}
\hline$n=500, \delta=25$ & \multicolumn{5}{c}{ Parameter quality (MSE and bias) } \\
\hline$\sigma^{2}$ & $\delta$ (O’Brien) & $\delta$ (Bayes) & $\beta_{0}$ & $\beta_{1}$ & $\sigma^{2}$ \\
\hline \multirow{2}{*}{0.01} & 0.01955 & 0.00543 & 0.00004 & 0.00008 & 0.000004 \\
& $(-0.10166)$ & $(0.00038)$ & $(-0.00033)$ & $(0.00004)$ & $(0.00005)$ \\
\hline \multirow{2}{*}{0.25} & 0.02108 & 0.00577 & 0.00097 & 0.00186 & 0.00026 \\
& $(-0.09609)$ & $(0.00138)$ & $(-0.00072)$ & $(0.00085)$ & $(0.00087)$ \\
\hline \multirow{2}{*}{1} & 0.04349 & 0.02236 & 0.00382 & 0.00773 & 0.0039 \\
& $(-0.08043)$ & $(0.00178)$ & $(-0.00014)$ & $(0.00174)$ & $(0.00499)$ \\
\hline \multirow{2}{*}{4} & 0.34816 & 0.23815 & 0.01683 & 0.03329 & 0.06341 \\
& $(-0.06625)$ & $(-0.00952)$ & $(-0.00583)$ & $(0.0158)$ & $(0.03492)$ \\
\hline \multirow{2}{*}{9} & 1.90617 & 1.41844 & 0.03879 & 0.07481 & 0.31347 \\
& $(-0.06601)$ & $(-0.00667)$ & $(-0.01006)$ & $(0.01655)$ & $(0.04772)$ \\
\hline
\end{tabular}

Table 4.3: Quality of cutpoints and regression parameters using a univariate linear regression model, when $X_{1} \in[0,50], \beta_{0}=1, \beta_{1}=-2, \delta_{1}=25, \sigma^{2}=0.01,0.25,1,4,9$ and $n=500$.

In each box the first row presents the $\widehat{\mathrm{MSE}}$ of the estimates and in the second row the corresponding bias is reported within parentheses.

\begin{tabular}{ccccccc}
\hline$\delta_{1}=25, \delta_{2}=25, \sigma^{2}=1$ & \multicolumn{7}{c}{ Parameter quality (MSE and bias) } \\
\hline $\mathrm{n}$ & $\delta_{1}$ & $\delta_{2}$ & $\beta_{0}$ & $\beta_{1}$ & $\beta_{2}$ & $\sigma^{2}$ \\
\hline \multirow{2}{*}{50} & 2.84054 & 3.58429 & 0.07004 & 0.09654 & 0.09607 & 0.05326 \\
& $(0.00984)$ & $(0.05485)$ & $(-0.00589)$ & $(0.02788)$ & $(-0.01524)$ & $(0.06038)$ \\
\hline \multirow{2}{*}{100} & 0.6336 & 0.71235 & 0.03038 & 0.04043 & 0.03997 & 0.02544 \\
& $(0.01561)$ & $(0.01159)$ & $(0.00419)$ & $(0.00276)$ & $(-0.01406)$ & $(0.02154)$ \\
\hline \multirow{2}{*}{200} & 0.13543 & 0.16293 & 0.01604 & 0.02059 & 0.0209 & 0.01117 \\
& $(0.00329)$ & $(0.00329)$ & $(0.00081)$ & $(0.00271)$ & $(-0.00767)$ & $(0.0169)$ \\
\hline \multirow{2}{*}{500} & 0.02425 & 0.02526 & 0.00603 & 0.00773 & 0.00825 & 0.00393 \\
& $(0.01097)$ & $(0.00454)$ & $(0.00037)$ & $(0.00081)$ & $(-0.00555)$ & $(0.00433)$ \\
\hline \multirow{2}{*}{1000} & 0.0065 & 0.00576 & 0.0028 & 0.00411 & 0.0039 & 0.00197 \\
& $(-0.00145)$ & $(-0.00073)$ & $(0.00063)$ & $(0.00041)$ & $(0.00082)$ & $(0.00346)$ \\
\hline
\end{tabular}

Table 4.4: Quality of cutpoints and regression parameters using a bivariate linear regression model, when $X_{1} \in[0,50], X_{2} \in[0,50], \beta_{0}=1, \beta_{1}=-2, \beta_{2}=2, \delta_{1}=25$, $\delta_{2}=25, \sigma^{2}=1$ and $n=50,100,200,500,1000$.

In each box the first row presents the $\widehat{\mathrm{MSE}}$ of the estimates and in the second row the corresponding bias is reported within parentheses. 


\begin{tabular}{ccccccc}
\hline$n=500, \sigma^{2}=1$ & \multicolumn{7}{c}{ Parameter quality $($ MSE and bias) } \\
\hline$\left(\delta_{1}, \delta_{2}\right)$ & $\delta_{1}$ & $\delta_{2}$ & $\beta_{0}$ & $\beta_{1}$ & $\beta_{2}$ & $\sigma^{2}$ \\
\hline \multirow{2}{*}{$(25,25)$} & 0.02425 & 0.02526 & 0.00603 & 0.00773 & 0.00825 & 0.00393 \\
& $(0.01097)$ & $(0.00454)$ & $(0.00037)$ & $(0.00081)$ & $(-0.00555)$ & $(0.00433)$ \\
\hline \multirow{2}{*}{$(30,30)$} & 0.02149 & 0.02562 & 0.00478 & 0.00906 & 0.00819 & 0.00403 \\
& $(-0.00408)$ & $(-0.00263)$ & $(-0.00187)$ & $(0.00023)$ & $(-0.00352)$ & $(0.00617)$ \\
\hline \multirow{2}{*}{$(35,35)$} & 0.02551 & 0.02626 & 0.00337 & 0.00934 & 0.00888 & 0.00411 \\
& $(-0.00334)$ & $(0.00217)$ & $(0.00057)$ & $(-0.00274)$ & $(-0.00015)$ & $(0.00593)$ \\
\hline \multirow{2}{*}{$(40,40)$} & 0.02329 & 0.02618 & 0.00295 & 0.01348 & 0.01267 & 0.00404 \\
& $(0.00251)$ & $(-0.00415)$ & $(-0.00101)$ & $(-0.00568)$ & $(0.00225)$ & $(0.00445)$ \\
\hline \multirow{2}{*}{$(45,45)$} & 0.02168 & 0.02316 & 0.00231 & 0.02263 & 0.02278 & 0.00409 \\
& $(-0.00668)$ & $(-0.00559)$ & $(-0.00166)$ & $(0.01309)$ & $(-0.00244)$ & $(0.00445)$ \\
\hline
\end{tabular}

Table 4.5: Quality of cutpoints and regression parameters using a bivariate linear regression model, when $X_{1} \in[0,50], X_{2} \in[0,50], \beta_{0}=1, \beta_{1}=-2, \beta_{2}=2$, $\left(\delta_{1}, \delta_{2}\right)=(25,25),(30,30),(35,35),(40,40),(45,45), \sigma^{2}=1$ and $n=500$.

In each box the first row presents the $\widehat{\mathrm{MSE}}$ of the estimate and in the second row the corresponding bias is reported within parentheses.

\begin{tabular}{ccccccc}
\hline$n=500, \delta_{1}=25, \delta_{2}=25$ & \multicolumn{7}{c}{ Parameter quality (MSE and bias) } \\
\hline$\sigma^{2}$ & $\delta_{1}$ & $\delta_{2}$ & $\beta_{0}$ & $\beta_{1}$ & $\beta_{2}$ & $\sigma^{2}$ \\
\hline \multirow{2}{*}{0.01} & 0.00466 & 0.00519 & 0.000006 & 0.000008 & 0.000008 & 0.000001 \\
& $(0.00207)$ & $(0.00078)$ & $(-0.00019)$ & $(0.00012)$ & $(0.00029)$ & $(0.00003)$ \\
\hline \multirow{2}{*}{0.25} & 0.0065 & 0.00697 & 0.00155 & 0.00202 & 0.00209 & 0.00025 \\
& $(-0.00267)$ & $(-0.00099)$ & $(0.0016)$ & $(0.00019)$ & $(-0.00185)$ & $(0.00025)$ \\
\hline \multirow{2}{*}{1} & 0.02425 & 0.02526 & 0.00603 & 0.00773 & 0.00825 & 0.00393 \\
& $(0.01097)$ & $(0.00454)$ & $(0.00037)$ & $(0.00081)$ & $(-0.00555)$ & $(0.00433)$ \\
\hline \multirow{2}{*}{4} & 0.25289 & 0.26982 & 0.02707 & 0.03213 & 0.03642 & 0.07056 \\
& $(0.00685)$ & $(0.01054)$ & $(0.0077)$ & $(0.00162)$ & $(-0.01604)$ & $(0.03399)$ \\
\hline \multirow{2}{*}{9} & 1.50236 & 1.4011 & 0.05492 & 0.06989 & 0.07469 & 0.33771 \\
& $(-0.00628)$ & $(-0.04681)$ & $(0.00081)$ & $(0.01139)$ & $(-0.01388)$ & $(0.06543)$ \\
\hline
\end{tabular}

Table 4.6: Quality of cutpoints and regression parameters using a bivariate linear regression model, when $X_{1} \in[0,50], X_{2} \in[0,50], \beta_{0}=1, \beta_{1}=-2, \beta_{2}=2, \delta_{1}=25$, $\sigma^{2}=0.01,0.25,1,4,9$ and $n=500$.

In each box the first row presents the $\widehat{\mathrm{MSE}}$ of the estimate and in the second row the corresponding bias is reported within parentheses.

For each parameter we obtain the estimated Mean Squared Error ( $\widehat{\mathrm{MSE}})$ of its estimates (from $\mathrm{K}=1000$ simulated data sets) as: 


$$
\widehat{\mathrm{MSE}}=\frac{1}{k} \sum_{i=1}^{k}\left(\theta-\hat{\theta}_{i}\right)^{2},
$$

where, $\theta$ is either $\delta_{1}, \delta_{2}, \beta_{0}, \beta_{1}, \beta_{2}, \sigma^{2}$. The simulation study is performed with the R software, version 3.2.1 [128]. The results for models with one covariate and two covariates are tabulated in Tables $4.1-4.3$ and Tables 4.4 - 4.6, respectively.

In the univariate setting we compare the Bayesian approach with O'Brien's [92] approach as a classical method. However, to the best of our knowledge there is no method available dealing with the multivariate dichotomization with respect to a continuous outcome, to compare with the Bayesian approach.

From the results of Tables 4.1 - 4.6, we observe that the performance of the Bayesian approach in estimating the cutpoints and the regression parameters is quite satisfactory with very small average biases and $\widehat{\mathrm{MSEs}}$ relative to the parameter values. Tables 4.1 and 4.4 clearly show that the $\widehat{\mathrm{MSE}}$ decreases as the sample size increases, for both the cutpoints and regression parameters. In the univariate setting, the Bayesian approach clearly outperforms O'Brien's method with $\widehat{\mathrm{MSEs}}$ of almost half of those using O'Brien's method and smaller average biases. For the given sample sizes, Tables 4.2 and 4.5 show that the cutpoints have almost the same $\widehat{\mathrm{MSEs}}$ when the true cutpoints range from the median to the $90^{\text {th }}$ percentile of the covariates. Note that the proposed Bayesian approach still performs twice as good as O'Brien's method in terms of the $\widehat{\mathrm{MSE}}$, for the range of true cutpoint. From Tables 4.3 and 4.6, we observe that the $\widehat{\mathrm{MSES}}$ obtained using the proposed Bayesian approach are close to zero when $\sigma^{2}$ is very small. As we expected $\widehat{\mathrm{MSE}}$ increases as $\sigma^{2}$ increases. From Table 4.3 we also see that the proposed Bayesian approach performs better than O'Brien's method in terms of $\widehat{\mathrm{MSE}}$ and average bias for different values of $\sigma^{2}$. Note that we could also use more informative priors for the parameters of the model which would then give better Bayes estimates in terms of $\widehat{\mathrm{MSE}}$.

\subsection{Application to the TIME-CHF Study}

The use of biomarkers, as diagnostic and prognostic factors in clinical decision making, is increasingly important in many areas of medicine. For instance, N-terminal pro-B-type Natriuretic Peptide (NT-proBNP) is a very well-known prognostic biomarker of heart failure and is used to exclude Heart Failure (HF) in patients presenting themselves with signs and symptoms of heart failure, both in the outpatient setting and in the emergency room [103]. The NT-proBNP level is normally very low in healthy individuals, it is increased in HF, and it correlates well with ventricular wall stress and the severity of HF [58, 80].

In this section we apply the proposed Bayesian approach to estimate the cutpoints for 
covariates in the TIME-CHF study with respect to NT-proBNP as the outcome variable. The design of the TIME-CHF study has been described in detail in Section 2.1. The analysis in this section includes 622 heart failure patients from the TIME-CHF study.

The covariates of which we are interested to find the cutpoints are: the BMI (Body Mass Index) and two biomarkers: SST2 (soluble ST2) and Mimecan at the baseline visit. In Table 4.7, we have the descriptive summaries of these covariates.

\begin{tabular}{ccccc}
\hline Variable & Mean & Standard deviation & Median & (Min, Max) \\
\hline BMI $\left(\mathrm{kg} / \mathrm{m}^{2}\right)$ & 25.4985 & 4.1987 & 25.014 & $(15.058,37.716)$ \\
SST2 $(\mathrm{ng} / \mathrm{ml})$ & 45.2249 & 32.0763 & 35.8423 & $(12.223,302.45)$ \\
Mimecan $(\mathrm{ng} / \mathrm{ml})$ & 132.2549 & 59.4857 & 119.04 & $(20.34,349.03)$ \\
\hline
\end{tabular}

Table 4.7: Descriptive summaries of the three covariates, BMI, SST2 and Mimecan.

In order to estimate the cutpoints for these covariates using the proposed Bayesian method of this chapter, we assume that the covariates in dichotomized form have a relationship with NT-proBNP (as an outcome variable) through a multivariate linear regression model. For notational convenience, we denote:

$Y=$ NT-proBNP (in logarithmic form)

$X_{1}=\mathrm{BMI}$

$X_{2}=$ SST2

$X_{3}=$ Mimecan

Following Equation (4.1) and the notations in Section 4.2, we assume that the dichotomized covariates are related to NT-proBNP through the following multivariate regression model:

$$
Y_{i}=\beta_{0}+\beta_{1} Z_{i 1}+\beta_{2} Z_{i 2}+\beta_{3} Z_{i 3}+\epsilon_{i}
$$

where $Z_{i l}$ is the dichotomized covariate obtained from $X_{i l}, l=1, \ldots, 3, i=1, \ldots, 622$, at cutpoints $\delta_{1}, \delta_{2}$ and $\delta_{3}$, respectively.

Assuming the non-informative prior in (4.10), where $\boldsymbol{\beta}=\left(\beta_{0}, \beta_{1}, \beta_{2}, \beta_{3}\right)$, and $\delta_{1} \sim$ $\mathrm{U}(15.058,37.716), \delta_{2} \sim \mathrm{U}(12.223,302.45)$ and $\delta_{3} \sim \mathrm{U}(20.34,349.03)$, we generate $1,000,000$ Gibbs samples to estimate the unknown parameters with a burn-in period of size 500,000. Geweke's convergence diagnostic test [39] confirms the convergence of generated Gibbs samples at a 5 percent level of significance for $\beta_{0}, \beta_{1}, \beta_{2}, \beta_{3}, \delta_{1}, \delta_{2}$ and $\delta_{3}$ with p-values of $0.44398,0.69872,0.52921,0.37318,0.49566,0.47216$ and 0.53591 , 
respectively.

The Bayes estimates of the unknown parameters (under the SEL function) with their corresponding Standard Deviation (SD) and 95\% credible intervals are presented in Table 4.8 .

\begin{tabular}{cccc}
\hline Parameter & Bayes Estimates & SD & 95\% Credible interval \\
\hline$\beta_{0}$ & -0.06988 & 0.04698 & $(-0.16763,0.01839)$ \\
$\beta_{1}$ & -0.18026 & 0.04058 & $(-0.25831,-0.10268)$ \\
$\beta_{2}$ & 0.24356 & 0.03435 & $(0.17582,0.31081)$ \\
$\beta_{3}$ & 0.23072 & 0.03344 & $(0.16502,0.29618)$ \\
$\delta_{1}$ & 23.18228 & 1.28176 & $(22.19415,26.86243)$ \\
$\delta_{2}$ & 41.53612 & 3.52424 & $(33.30335,49.53026)$ \\
$\delta_{3}$ & 135.2686 & 6.73549 & $(118.3009,146.763)$ \\
\hline
\end{tabular}

Table 4.8: Bayes estimates of regression parameters and cutpoints.

From Table 4.8 the estimated cutpoint for BMI, SST2 and Mimecan are 23.18228 $\mathrm{kg} / \mathrm{m}^{2}, 41.53612 \mathrm{ng} / \mathrm{ml}$ and $135.2686 \mathrm{ng} / \mathrm{ml}$, respectively. We observe that the effects of dichotomized SST2, Mimecan and BMI on NT-proBNP are significant since the credible intervals for $\beta_{1}, \beta_{2}$ and $\beta_{3}$ do not include zero. In addition, by applying the multivariate regression model with dichotomized covariates we observe that the Shapiro-Wilk normality test $[126]$ is not significant ( $p$-value $=0.5575$ ) which shows there is no reason to reject the assumption of normality.

Numerous studies have addressed the relationship between BMI and NT-proBNP or risk of $\mathrm{HF}$ and investigated the best cutpoint to explain these relationships [24, 64]. Recent studies have demonstrated that patients with underweight (BMI $<25 \mathrm{~kg} / \mathrm{m}^{2}$ ) have higher NT-proBNP and higher risk of HF [25]. This observation is known as the 'obesity paradox' [89]. The estimated coefficient for dichotomized BMI $\left(\beta_{1}=-0.18026\right)$ in Table 4.8 also confirms the negative association previously observed between BMI and NT-proBNP. We note that, the obtained credible interval of the estimated cutpoint for BMI (22.19415, 26.86243) also includes the recommended cutpoint of 25. Several studies have shown the positive association between SST2 and NT-proBNP [62] and have recommended the cutpoint of $35 \mathrm{ng} / \mathrm{ml}$ for SST2 [59]. It has been demonstrated that patients are at a higher risk of adverse events when SST2 levels are above $35 \mathrm{ng} / \mathrm{ml}[59,66]$. The estimated coefficient ( $\beta_{2}=0.24356$ ) in Table 4.8 confirms the positive association between SST2 and NT-proBNP. Moreover, the obtained credible interval of the estimated cutpoint for SST2 $(33.30335,49.53026)$ includes the previously recommended cutpoint of $35 \mathrm{ng} / \mathrm{ml}$. The level of SST2 (and consequently the cutpoint) depends on different factors including age and severity of heart failure. Given the high age of the population studied (older than 65 years), this may explain why the estimated cutpoint in our study is slightly higher than 
previously suggested. Mimecan is a very new biomarker for which to the best of our knowledge this is the first study to recommend a cutpoint.

\subsection{Discussion}

In this chapter we proposed a Bayesian approach to estimate cutpoints for continuous covariates assuming that the covariates in dichotomized form are related to an outcome variable through a univariate or a multivariate linear regression model. The proposed Bayesian approach has the great advantage that it can be applied in a multivariate setting. In the multivariate setting with the use of this Bayesian method we may dichotomize all or only a selected subset of the covariates. To the best of our knowledge this is the first method dealing with multivariate dichotomization with respect to a continuous outcome variable directly. Unlike most of the available methods, in using the Bayesian approach the estimated cutpoint is not limited to the observed values of the covariate and can take any value in the range of the covariate.

We applied our simulation study in univariate and bivariate setting considering the effect of sample sizes, true value of the cutpoint and the variance of the error terms. It showed that the proposed Bayesian approach provides good estimates of the true cutpoint even when the variance of the error term is relatively high (equal to 9). In addition, the simulation study showed that in a univariate setting, the Bayesian approach has better performance compared to O'Brien's method. However, the simulation study is only applied for a limited range of choices of the parameters. Therefore, it might be interesting to further investigate the performance of the proposed Bayesian approach for different choices of the parameters and models with more than two covariates. The noninformative prior performs very well in the simulation study. However, one might be interested to also see the performance of the other noninformative priors such as Bernardo's prior [14] and Jeffreys [56] prior.

To demonstrate the proposed approach with real data, we applied it to estimate the cutpoints for three markers in the TIME-CHF study, BMI, SST2 and Mimecan, with respect to NT-proBNP. We observed that the estimated cutpoints and associations of the dichotomized BMI and SST2 with NT-proBNP were well in line with the recommended cutpoints and previously observed associations in other studies. The recommended cutpoints in these other studies are mostly based on experience or on assessing the individual effect of the dichotomized biomarker on an outcome of interest. Moreover, these recommended cutpoints vary slightly from study to study, suggesting that more sophisticated methods for cutpoint selection are important. The cutpoint for a given covariate may also depend on other covariates. In contrast with other studies, with the use of the proposed Bayesian approach we estimated the cutpoints in a multivariate setting. We note that one might be interested to estimate the cutpoints as well in the presence of the other covari- 
ates or for more biomarkers. However, this should be done with caution due to the limited number of observations in the study and possible multicollinearity between the biomarkers which may lead to low accuracy of results. We also note that the estimated cutpoint for Mimecan should be validated in future studies since, to the best of our knowledge, this is the first study which investigated a cutpoint for Mimecan.

Finally we note that the inferences in this chapter are under the assumption of multivariate normality that may not be applicable to other distributions. Therefore, as a generalization of the approach in this chapter one may consider dichotomization using generalized linear regression models. 


\section{Generalized Ranking Accuracy for Logistic-GEE Models}

This chapter is based on: Davarzani, N., Peeters, R., Smirnov, E., Karel, J., Brunner-La Rocca, H.P. Ranking Accuracy for Logistic-GEE Models. In: Boström H., Knobbe A., Soares C., Papapetrou P. (eds), Advances in Intelligent Data Analysis XV. Proceedings of IDA 2016. Lecture Notes in Computer Science, Vol. 9897: 14-25. 


\begin{abstract}
Logistic Generalized Estimating Equations (logistic-GEE) models have been extensively used for analyzing clustered binary data. However, assessing the goodness-of-fit and the predictive performance of logistic-GEE models is problematic due to the fact that the outcomes are correlated within a cluster and no likelihood is available. In this chapter we address this issue and we propose a new performance measure for logistic-GEE models, which we call the Generalized Ranking Accuracy (GRA). We define GRA as the probability that a randomly selected instance with positive outcome is ranked higher than a randomly selected instance with negative outcome from another cluster. GRA can be used for assessing the goodness-of-fit and the predictive performance of logistic-GEE models, depending on the type of evaluation method (e.g. cross-validation, or hold-out). A simulation study is conducted to evaluate and compare GRA with other goodness-of-fit criteria when logistic-GEE models are trained on clustered data. In addition, we employ GRA as a model selection criterion in the TIME-CHF study.
\end{abstract}




\subsection{Introduction}

The Logistic Generalized Estimating Equations (logistic-GEE) model ${ }^{1}$ is a widely used model for analysing clustered binary data [47]. However, evaluating goodness-of-fit and predictive performance of the constructed logistic-GEE model still is a challenging area of research [31,32]. This is due to the fact that no likelihood is available and, moreover, the model's predicted probabilities are correlated within a cluster. In addition, the logisticGEE model disregards the unknown correlation between the outcomes within a cluster and treats it as a nuisance parameter and simply models the mean of outcomes on the population level [73].

In this chapter we address the problem of evaluating the logistic-GEE model and propose a new criterion as a goodness-of-fit criterion for this model. This problem has been considered by several authors (see e.g, [13, 31, 32, 49, 97, 98, 107, 142]). Several criteria and tests have been proposed for assessing the goodness-of-fit of logistic-GEE models. However, most of them have their own shortcomings making it impossible to have a commonly applicable and accepted criterion or test. In addition, to the best of our knowledge, so far, no predictive performance criterion has been proposed for the logisticGEE model in the literature. The new proposed criterion in this study, to some extent, can also be used for assessing the predictive performance of the logistic-GEE model. To put this research into perspective, we first describe relevant work, after which we propose our approach.

Barnhart and Williamson in [13] proposed a model-based and robust goodness-of-fit tests for logistic-GEE models. The method is based on partitioning the space of covariates into distinct regions and forming score statistics that are asymptotically distributed as chi-square random variables. The main disadvantage of this method is that the test statistics and the corresponding degrees of freedom depend on the subjective choice of cutpoints for covariate partitioning. Moreover, the authors mentioned that in order to ensure the asymptotic properties of the tests, each partitioned region should contain at least 10 clusters and only $25 \%$ of the regions should have less than 25 clusters. Therefore, applying this method might be problematic in studies such as TIME-CHF when we would like to have many continuous covariates in the model, or when sample sizes are small.

Pan in [98] introduced two goodness-of-fit statistics for logistic-GEE models: the Pearson chi-square statistic and an unweighted sum of squares statistic. These two statistics are based on comparing observed and predicted residual values. The major drawback of both statistics lies in the necessity of involving at least one continuous covariate in the model. In addition, the author mentioned that in practice the power of these statistics may be limited since these statistics are proposed to detect only general model departures.

\footnotetext{
${ }^{1}$ The formulation and usage of the logistic-GEE model are explained in detail in Section 2.4.
} 
Another criterion for assessing the goodness-of-fit of logistic-GEE models is a Kappalike classification statistic proposed by Williamson et al. in [142]. This Kappa-like statistic depicts how well the binary outcomes are predicted from the logistic-GEE model [142]. It takes the value of one if prediction is perfect and zero if the fitted model predicts no better than an intercept-only model. The disadvantage of the statistic is that for twoclass imbalanced data it usually tends to be close to zero, i.e., it states that the model is poorly fitted. Moreover, since no distribution of the statistic is given, interpretation of the statistic is not obvious. The authors made suggestions for interpreting the statistic, based on Landis and Koch's labeling of Kappa-like statistic values [70]. They recommended that a value from 0 to 0.20 indicates a poor fit, a value from 0.21 to 0.40 indicates a fair fit, a value from 0.41 to 0.60 indicates a good fit, and a value from 0.61 to 1.00 indicates an excellent fit. However, this interpretation might not be suitable for some sensitive studies. For instance, the value of 0.61 means that $39 \%$ of the sample evaluations is predicted wrong which in a clinical study would be a serious problem.

One of the well-established goodness-of-fit statistics for GEE models is a Quasilikelihood under the independence model Information Criterion (QIC) [97] which is an extension of Akiake's information criterion (AIC)[7]. QIC is recommended to be useed as a criterion for choosing between working correlation structures rather than as a modelselection criterion [49]. As an alternative, QICu (as an extension of QIC) is proposed to be used as a model-selection criterion [49], such that the model with smaller QICu is preferred. Since QICu is a function of both quasilikelihood (which depends on the size of the working dataset) and the number of estimated parameters in the model, it indicates the quality of a model relative to other models, fitted with the same data set. When using different data sets, the QICu values may be very different. To the best of our knowledge there is no way to normalize the QICu value which makes it impossible to use QICu as an absolute quality measure. Therefore, QICu is not an applicable criterion for comparing the goodness-of-fit of GEE models for different data sets, even with the same covariates. Moreover, QICu is only a goodness-of-fit criterion and it does not provide information on the predictive performance of the model, since it is based on the training data.

Each of the aforementioned goodness-of-fit test statistics and criteria for logistic-GEE models has at least one of the following shortcomings:

(a) restriction on the number and types of covariates in the model being evaluated,

(b) bias in case of two-class imbalanced data,

(c) difficulty of interpretation,

(d) a relative range of the criterion values (i.e., the range depends on the number of subjects and number of covariates in the model),

(e) inability to indicate the predictive performance of the model being evaluated. 
To propose a criterion that does not suffer from problems (a)-(e), we observe that:

(1) logistic-GEE models are trained on clustered data,

(2) logistic-GEE models provide probabilities for the outcomes to be positive,

The latter implies that logistic-GEE models provides scores (continuous range, between 0 and 1) over the instances. Thus, logistic-GEE models actually solve the bipartite ranking task for clustered data [3], see Section 2.4.1. The task is as follows: given labeled clustered data, find an ordering on the instances such that instances with positive outcomes (outcome $=1$ ) are ranked higher than instances with negative ones (outcome $=0){ }^{2}$ The standard measure for the quality of that ordering is the Ranking Accuracy (RA), see [3]. However, its applicability for logistic-GEE models is questionable, since it does not take into account the within-cluster correlation that might be present, and thus it can be invalid.

In this chapter we extend the concept of ranking accuracy to clustered data. We propose a new measure that we call the Generalized Ranking Accuracy (GRA) for models based on clustered binary data. It is defined as the probability that a randomly selected instance with positive outcome (positive instance) is ranked higher than a randomly selected instance with negative outcome (negative instance) from another cluster. The definition of GRA is such that it avoids using multiple correlated observations from the same cluster at the same time. We note that, (logistic-)GEE models also aim to disregard within-cluster correlation, to build a population-average model. Therefore, the GRA definition is chosen to be in line with the same concept.

We also propose a computationally efficient algorithm for GRA (Section 5.2.2). The algorithm employs only the model's predicted probabilities and the outcome values; i.e., it does not use any internal information from the model being used. This implies that GRA is applicable for logistic-GEE models as well as for any other valid model for bipartite ranking based on clustered data.

We show that GRA can be used both as a goodness-of-fit criterion and as a predictive performance criterion (Section 5.2.3). For the latter we propose a modification to the standard cross-validation method to make it applicable for clustered data.

When comparing GRA with the presented standard goodness-of-fit test statistics and criteria for logistic-GEE models we observe that GRA overcomes problems (a) to (e) given above. The main reasons are that: (1) GRA does not impose any restriction on the number and types of covariates in the models being evaluated; (2) GRA is not biased for binary imbalanced data (since it indicates class separation); (3) GRA is a probability that is fairly easy to interpret; (4) GRA has an absolute range; and (5) GRA can be used both as a goodness-of-fit criterion and as a predictive performance criterion.

\footnotetext{
${ }^{2}$ We note that a good solution for the bipartite ranking task implies a good solution for the binary classification task. However, the opposite is not always true [3].
} 
The rest of the chapter is organized as follows. In Section 5.2 we introduce GRA in full detail. We use experiments in Section 5.3 on simulated data to compare GRA as a goodness-of-fit criterion with a Kappa-like statistic, QICu, and RA, when evaluating logistic-GEE models. In addition, we compare GRA (using cross-validation) as a model selection criterion with QICu. In Section 5.4 we employ GRA as a model selection criterion in the TIME-CHF study and in Section 5.5 we conclude this chapter by discussing our results and highlighting the importance and advantages of the GRA.

\subsection{Generalized Ranking Accuracy}

In this section we define Generalized Ranking Accuracy (GRA) for models trained on clustered binary data and we explain how it relates to logistic-GEE models. We present an algorithm for computing the GRA together with a complexity analysis. Then we evaluate the obtained GRA both as a goodness-of-fit criterion and as a predictive performance criterion for logistic-GEE models.

\subsubsection{Definition and Computation of the GRA}

According to Eqn. (2.9) any logistic-GEE model is essentially a scoring classifier model [22]. Following the definitions and notations in Section 2.4, given a data set of $n$ clusters $\left\langle\boldsymbol{X}_{i}, \boldsymbol{Y}_{i}\right\rangle, i=1, \ldots, n$, each consisting of instances $\left(\boldsymbol{x}_{i t}, y_{i t}\right), t=1, \ldots, m_{i}$, a logisticGEE model provides a probability $\pi_{i t}$ for the outcome $Y_{i t}$ to be positive, given the vector $\boldsymbol{x}_{i t}, i=1, \ldots, n, t=1, \ldots, m_{i}$. This probability $\pi_{i t}$ is a value in $[0,1]$, which can be interpreted as a score: a higher score implies a higher probability to be dealing with a positive outcome, and a good model is expected to provide higher scores for instances with a positive outcome than for instances with a negative outcome. The standard measure for such a comparison is RA [3]. It is defined as the probability that a randomly selected instance with positive outcome (positive instance) is ranked higher by the scoring model than a randomly selected instance with negative outcome (negative instance).

To apply the concept of the RA to a logistic-GEE model, or any scoring model trained on clustered binary data, we introduce GRA. The GRA aims to measure RA, by avoiding to compare rankings within a cluster - as they are known to be correlated (but in an unknown way) which may distort the validity of the comparison.

Definition 1. The GRA of a scoring model trained for clustered data is defined as the probability that a randomly selected positive instance (from an arbitrary cluster) ranks higher than the randomly selected negative instance from another cluster.

The GRA is easy to interpret, since it is a probability (i.e., it ranges between 0 and 1). The value 1.0 indicates perfect ranking on the given data (i.e., all positive instances are 
ranked higher than all negative instances from another cluster); and the value 0.0 indicates the opposite scenario. The value 0.5 corresponds to a noninformative model.

Below we introduce the exact formula for GRA. Following the GRA definition, provided above, first for any positive instance $\left(\boldsymbol{x}_{i t}, y_{i t}\right)$ i.e., with $y_{i t}=1$, we determine the number $D_{i t}$ of negative instances from other clusters:

$$
D_{i t}=\sum_{j=1, j \neq i}^{n} \sum_{s=1}^{m_{j}} I\left\{y_{j s}=0\right\}, i=1, \ldots, n, t=1, \ldots, m_{i}
$$

where $I$ is the indicator function having the value 1 when $y_{j s}=0$, otherwise 0 .

The number $D_{i t}$ can be interpreted as the number of pairs consisting of instances with a particular positive outcome, $y_{i t}$, from cluster $i$ and one negative instance from other clusters. We note that this number stays the same for each positive instance in cluster $i$. This implies that the number $D_{i}$ of pairs consisting of instances, one positive from cluster $i$, one negative from another cluster, is equal to:

$$
D_{i}=\sum_{t=1}^{m_{i}} D_{i t} I\left\{y_{i t}=1\right\}
$$

Then the total number of pairs consisting a positive and a negative instances not from the same cluster, is equal to:

$$
D=\sum_{i=1}^{n} D_{i}
$$

Now following the GRA definition, we are interested to know how many of these $D$ pairs correctly are ranked by the logistic-GEE model. We assume that for each instance $\left(\boldsymbol{x}_{i t}, y_{i t}\right)$ the logistic-GEE model provides a (score) probability of being positive, $\pi_{i t}$, $i=1, \ldots, n, t=1, \ldots, m_{i}$. We rank instance $\left(\boldsymbol{x}_{i t}, y_{i t}\right)$ according to $\pi_{i t}$. For any positive instance $\left(\boldsymbol{x}_{i t}, y_{i t}\right)$, we compute $C_{i t}$ as the number of correct pairs, produced by the ranking through combining with all negative instances $\left(\boldsymbol{x}_{j s}, y_{j s}\right)$ from all other clusters $j$, such that $j \neq i$. In case of equal scores, the probability of properly ranking the instances is taken to be $1 / 2$. The number $C_{i t}$ then is given by:

$$
C_{i t}=\sum_{j=1, j \neq i}^{n} \sum_{s=1}^{m_{j}}\left(I\left\{\pi_{i t}>\pi_{j s}\right\}+\frac{1}{2} I\left\{\pi_{i t}=\pi_{j s}\right\}\right) I\left\{y_{j s}=0\right\}
$$

The number $C_{i t}$ does not stay the same for each positive instance in cluster $i$. Hence, the number of correctly ranked pairs of a positive instance from cluster $i$ with negative 
instance from another cluster $j(j \neq i)$ is equal to:

$$
C_{i}=\sum_{t=1}^{m_{i}} C_{i t} I\left\{y_{i t}=1\right\} .
$$

Thus, $C$ as the number of correctly ranked pairs of positive and negative instances from distinct clusters is equal to:

$$
C=\sum_{i=1}^{n} C_{i}
$$

Finally the GRA is given by,

$$
\text { GRA }=\frac{C}{D}
$$

\subsubsection{Algorithm for GRA}

Below, in Algorithm 1, we provide an algorithm for efficient computation of the GRA. The input of the algorithm consists of $n$ clusters $\left\langle\boldsymbol{x}_{i}, \boldsymbol{y}_{i}\right\rangle$ and their corresponding vectors $\boldsymbol{\pi}_{i}$ of probabilities $\pi_{i t}, i=1, \ldots, n, t=1, \ldots, m_{i}$, computed by a logistic-GEE model. The output of the algorithm is the GRA induced by the probabilities $\pi_{i t}$ with respect to the instances $\left(\boldsymbol{x}_{i t}, y_{i t}\right)$.

The algorithm consists of two parts: an initialization part (steps 1-23) and a computation part (steps 24-43). During the initialization a list $L$ of outcome-probability tuples is set to empty. The algorithm scans sequentially the clusters and the probability vectors to add all the outcome-probability tuples $\left\langle y_{i t}, \pi_{i t}\right\rangle$ to the list $L$. When the list $L$ is being handled, the statistics imposed by the outcomes $y_{i t}$ are being computed: the numbers $m_{i}^{+}$ and $m_{i}^{-}$of the positive and negative instances for each cluster $i$ and the total number $m^{-}$ of negative instances. These numbers are used to calculate the number $D_{i}$ for each cluster $i$ (see formula (5.2)) and total number $D$ (see formula (5.3)).

The algorithm completes the initialization by setting statistics related to the ordering imposed by probabilities $\pi_{i t}$. For each cluster $i$ it sets two statistics: (1) the number $C_{i}$ (see formula (5.5)), and (2) a counter $H_{i}$ that represents the number of negative instances in cluster $i$ that can be potentially paired with positive instances from any other cluster if the probabilities associated with those outcomes do not exceed a certain probability. The number $C_{i}$ receives initially a value of 0 and the counter $H_{i}$ receives the value of $m_{i}^{-}$. Once the counters $H_{i}$ are determined, $i=1, \ldots, n$, the algorithm computes their sum $H$ (initially set equal to $\mathrm{m}^{-}$).

The computation part starts with sorting the list $L$. The list is sorted on three levels. First, the list is sorted in a decreasing order of probability values $\pi_{i t}$. If they are ties, the outcome-probability tuples with the same probability $\pi_{i t}$ are sorted in an increasing 
order of the cluster number. Again, if they are ties, the outcome-probability tuples with the same cluster number $i$ are sorted in a decreasing order of the outcomes $y_{i t}$.

Once the list $L$ is sorted, the algorithm sequentially scans $L$ to compute the number $C_{j}$ (see formula (5.5)) for each cluster $j$. For any distinctive probability $\pi_{i t}$, in the decreasing order provided in $L$, the number $C_{j}$ receives an update equal to:

$$
P_{j}\left(H-H_{j}\right)-\frac{P_{j}\left(Q-Q_{j}\right)}{2}
$$

where,

- $Q$ is the number of negative instances whose probabilities $\pi_{k s}$ equal to $\pi_{i t}$;

- $P_{j}$ is the number of positive outcomes from cluster $j$ whose probabilities $\pi_{j s}$ equal to $\pi_{i t}$;

- $Q_{j}$ is the number of negative outcomes from cluster $j$ whose probabilities $\pi_{j s}$ equal to $\pi_{i t}$.

The term $P_{j}\left(H-H_{j}\right)$ in the expression (5.8) is the total number of positive-negative pairs that can be formed by positive outcomes from cluster $j$ whose probabilities $\pi_{j s}$ equal to $\pi_{i t}$ and negative outcomes from other clusters with probabilities less than or equal to $\pi_{i t}$. The term $P_{j}\left(Q-Q_{j}\right)$ in the expression (5.8) is the total number of positivenegative pairs that can be formed by positive outcomes from cluster $j$ whose probabilities $\pi_{j s}$ equal to $\pi_{i t}$ and negative outcomes from other clusters with probabilities equal to $\pi_{i t}$. We take only half of the term $P_{j}\left(Q-Q_{j}\right)$, since in this case, the probability of properly ranking the outcomes is $\frac{1}{2}$. Thus, the expression (5.8) represents an update for forming the number $C_{j}$ when we aim at estimating an averaged GRA.

Once all the numbers $C_{i}$ are computed, the algorithm computes number $C$ (see formula (5.6)) and then outputs the GRA (see formula (5.7)).

The algorithm for GRA is computationally efficient. Its space complexity is $O(\mathrm{~nm})$, where $n$ is the number of clusters and $m$ is the size of the clusters. The time complexity is $O\left(n m \log _{2}(n m)\right)$. To derive the time complexity of the algorithm for GRA we analyze the steps of the algorithm that involve sorting or search; i.e., the most computationally expensive steps. Step 24 executes sorting on three levels. On the first level the time complexity is $O\left(n m \log _{2}(n m)\right)^{3}$. On the second level the time complexity is $O\left(p \frac{n m}{p} \log _{2}\left(\frac{n m}{p}\right)\right)=O\left(n m \log _{2}\left(\frac{n m}{p}\right)\right)$, where $p$ is the number of distinctive probabilities $\pi_{i t}$. On the third level the time complexity is $O\left(n p \frac{n m}{n p} \log _{2}\left(\frac{n m}{n p}\right)\right)=O\left(n m \log _{2}\left(\frac{m}{p}\right)\right)$. Thus, the time complexity of step 24 is $O\left(n m \log _{2}(n m)\right)$.

\footnotetext{
${ }^{3}$ We assume usage of efficient sorting algorithms like merge sort.
} 


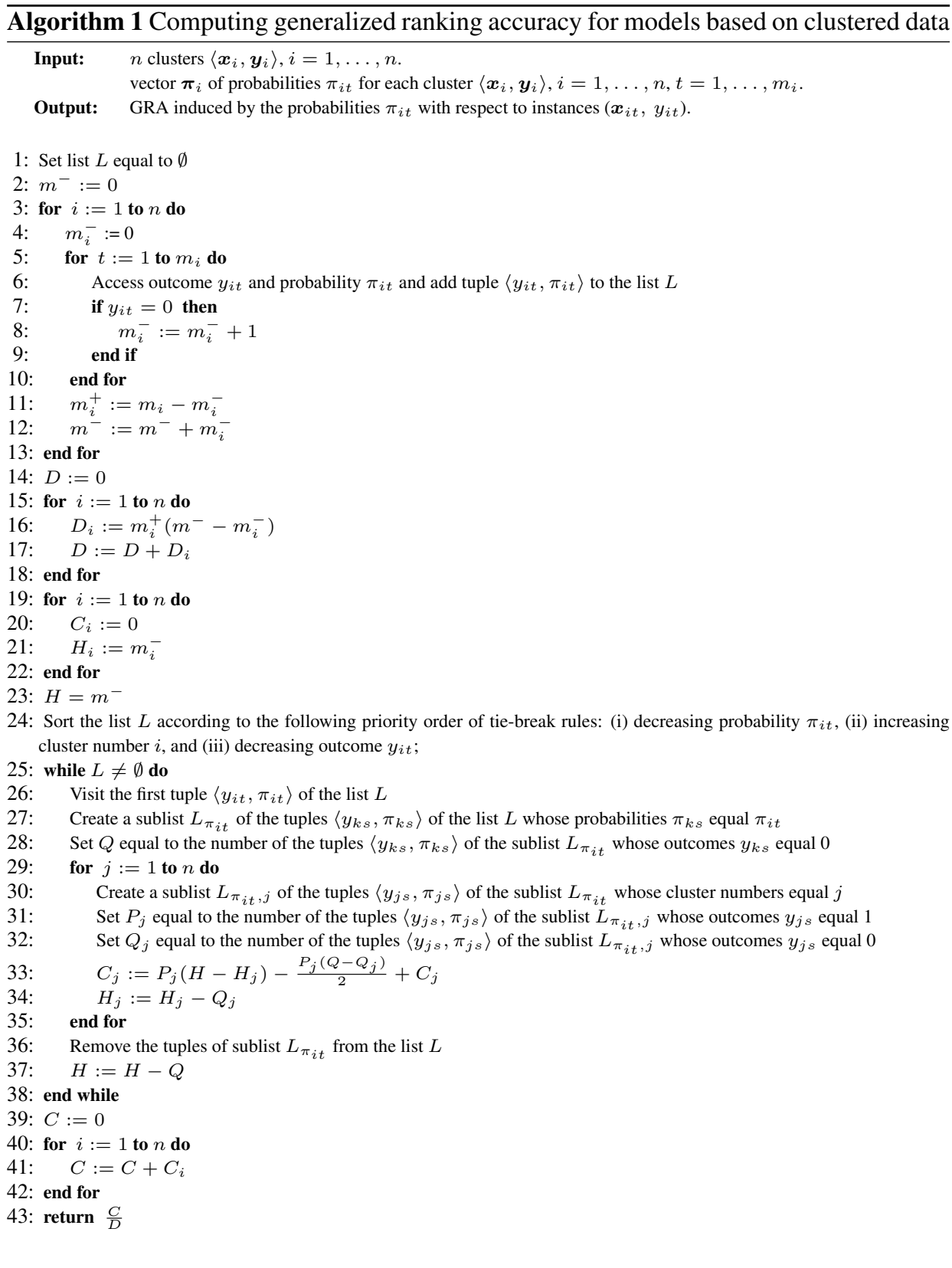

The steps $27,28,30,31$, and 32 perfom binary search due to the fact that the list $L$ is sorted on three levels. The time complexity of step 27 is $O\left(p \log _{2}(n m)\right)$, the time 
complexity of step $28^{4}$ is $O\left(p\left(\log _{2}\left(\frac{n m}{p}+n \log _{2}\left(\frac{n m}{p n}\right)\right)\right)=O\left(p\left(\log _{2}\left(\frac{n m}{p}+n \log _{2}\left(\frac{m}{p}\right)\right)\right)\right.\right.$, the time complexity of step 30 is $O\left(p n \log _{2}\left(\frac{n m}{p}\right)\right)$, and the time complexity of steps 31 and 32 is $O\left(p n \log _{2}\left(\frac{m}{p}\right)\right)$. Thus, the time complexity of the search steps is determined by the sum of the time complexities of steps 27 to 30 .

From the derivations provided above we observe that the time complexity of the sorting step 24 prevails that of the search steps 27, 28, 30, 31, and 32 . Thus, we may conclude that the time complexity of the algorithm for GRA is indeed $O\left(n m \log _{2}(n m)\right)$.

\subsubsection{Goodness-of-fit and Predictive Performance}

The proposed GRA can be used both as a criterion of the model's goodness-of-fit and as a criterion of the model's predictive performance. If a logistic-GEE model has been trained and tested on the same data, then GRA acts as a goodness-of-fit criterion. In this case GRA estimates how the logistic-GEE model fits the data when only combinations of uncorrelated observations are taken into account.

If the GRA is computed on the test data (on which the model was not trained), then the GRA acts as a predictive performance criterion for the model [100].

We note that the GRA, to the best of our knowledge, is the first criterion proposed for usage to assess a logistic-GEE model's predictive performance. There are several methods for computing the GRA as a predictive performance criterion, such as by combining it with the hold-out method, $k$-fold cross-validation, etc. In this thesis we employ the $k$-fold cross-validation method due to its relatively lower variance of the resulting estimates [15]. The $k$-fold cross-validation method for clustered data should be designed such that all the instances belonging to one and the same cluster, are either all in the test set or all in the training set. This avoids training and testing with correlated data. This method operates as follows. First, the data is split randomly into $k$ disjoint sets (folders) of clusters. Then, for each of the $k-1$ folders we train a logistic-GEE model (or any of the model trained on clustered data), and test this model on the remaining folder. For each test instance we estimate the probability $\pi_{i t}$ from the model according to Eqn. (2.9) and in this way we form the vector $\boldsymbol{\pi}_{i}$. When this vector has been formed for all the clusters we can run the algorithm for GRA. The GRA computed using the cross-validation method we denote as GRA-CV.

We note that the extreme version of the $k$-fold cross-validation method is cluster-out cross-validation when each fold contains a single cluster and $k$ equals the number of clusters.

\footnotetext{
${ }^{4}$ Note that step 28 involves double binary search.
} 


\subsection{Simulation study}

To evaluate various aspects of the usefulness of GRA, in this section we carry out a simulation study to assess GRA as a goodness-of-fit criterion and as a model-selection criterion for logistic-GEE models. We do this through the following comparisons and evaluations. We first compare GRA with a Kappa-like classification statistic [142] as goodness-of-fit criterion in the presence of two-class imbalanced and balanced data. Then we compare GRA and QICu [49]. As model selection criteria, we study both criteria as a function of logistic-GEE model complexity and we compare them in the context of model selection. Finally, we assess and compare GRA and the conventional RA.

\subsubsection{Data generation}

The first step of our simulation study is to generate a data set. Below we explain how to generate a data set that contains $n$ clusters of sizes $m_{i}, i=1, \ldots, n$, with correlated binary outcomes. Assume the $i^{\text {th }}$ subject is represented by a cluster of $m_{i}$ observations such that the $t^{\text {th }}$ observation, $t=1, \ldots, m_{i}$ is given with $p$ covariates $X_{i t 1}, \ldots, X_{i t p}$ in $\mathbb{R}$ and a binary outcome variable $Y_{i t}$. We generate the $p^{\text {th }}$ covariate for the $i^{\text {th }}$ subject $\boldsymbol{X}_{i}^{(p)}=\left(X_{i 1 p}, \ldots, X_{i m_{i} p}\right)$ from a known distribution. Then we assume that the marginal model generating the data is given by:

$$
\log \left(\frac{\pi_{i t}}{1-\pi_{i t}}\right)=\beta_{0}+\boldsymbol{\beta} \boldsymbol{X}_{i t}, i=1, \ldots, n, t=1, \ldots, m_{i},
$$

where $\boldsymbol{X}_{i t}=\left(X_{i t 1}, \ldots, X_{i t p}\right)^{\prime}, \pi_{i t}=\mathrm{E}\left(Y_{i t} \mid \boldsymbol{x}_{i t}\right)=\operatorname{Pr}\left(Y_{i t}=1 \mid \boldsymbol{x}_{i t}\right), i=1, \ldots, n$, $t=1, \ldots, m_{i}, \beta_{0}$ is the population averaged intercept term and $\boldsymbol{\beta}=\left(\beta_{1}, \ldots, \beta_{p}\right)$ is the vector of population averaged coefficients.

Here, we would like to generate outcomes $Y_{i t}$ in $\{0,1\}$ with probability $\pi_{i t}, i=$ $1, \ldots, n, t=1, \ldots, m_{i}$, for the outcome 1 . However, within each cluster $i$ we also want to achieve a specified correlation (with zero correlation between different clusters).

Now, assume that $\operatorname{corr}\left(Y_{i t}, Y_{i h}\right)=\eta_{t h}, 1 \leq t, h \leq m_{i}, t \neq h$, where $\eta_{t h}$ is given. Then we can generate binary outcomes $Y_{i 1}, \ldots, Y_{i m_{i}}$ with this specified correlation structure using the approach proposed in [30] considering the constraint that $\max \left(-\sqrt{\left(\pi_{i t} \pi_{i h} / q_{i t} q_{i h}\right)},-\sqrt{\left(q_{i t} q_{i h} / \pi_{i t} \pi_{i h}\right)}\right)<\eta_{t h}<\min \left(\sqrt{\left(\pi_{i t} q_{i h} / q_{i t} \pi_{i h}\right)}, \sqrt{\left(q_{i t} \pi_{i h} / \pi_{i t} q_{i h}\right)}\right)$, where $q_{k s}=1-\pi_{k s}$. We used the R package "SimCorMultRes" [132] to implement this.

\subsubsection{GRA versus Kappa-like Classification Statistic}

In this subsection we present a comparison of the GRA with a Kappa-like classification statistic (proposed by Williamson et al. [142]) as goodness-of-fit criteria for a logisticGEE model trained on simulated data. 
We generated data sets with $n=25,50,75,100,500,1000$ clusters and cluster sizes $m=2,5,10,20$ with exchangeable correlation structure (correlation equal to 0.3 ). For a given $n$ and $m$ we used the model in Eqn. (5.9) with two covariates where each is distributed as uniform( 0,1$)$ (using R package "SimCorMultRes").

In order to having two-class imbalanced data, we chose the regression coefficients $\beta_{0}=0, \beta_{1}=1$ and $\beta_{2}=-10$, which led to data sets with a proportion of positive instances between $8 \%$ and $12 \%$.

After fitting the logistic-GEE model we obtained GRA and Kappa-like values to evaluate the goodness-of-fit of model. This process was replicated 1000 times and Table 5.1 presents the averages of GRA and Kappa-like values.

We repeated this process in the presence of two-class balanced data sets with a proportion of positive instances between $46 \%$ to $54 \%$ by choosing $\beta_{1}=10$ and $\beta_{2}=-10$. The results are given in Table 5.2.

The results in Table 5.1 indicate that, in the case of two-class imbalanced data, after fitting the correct logistic-GEE models, GRA indicates an excellent fit of the models. Instead, the Kappa-like statistic indicates either a poor or a fair fit of the models (based on Landis and Koch's labeling of the Kappa-like statistic interpretation [70]). The results in Table 5.2 indicate that in the case of two-class balanced data, the Kappa-like statistic, just the same as GRA, then shows an excellent fit of the models.

\begin{tabular}{cccccccc}
\hline $\mathrm{m}$ & & $\mathrm{n}=25$ & $\mathrm{n}=50$ & $\mathrm{n}=75$ & $\mathrm{n}=100$ & $\mathrm{n}=500$ & $\mathrm{n}=1000$ \\
\hline \multirow{2}{*}{2} & GRA & 0.96084 & 0.95992 & 0.95811 & 0.95939 & 0.95988 & 0.95936 \\
& Kappa-like & 0.29808 & 0.25663 & 0.24101 & 0.23035 & 0.20785 & 0.20945 \\
\hline \multirow{2}{*}{5} & GRA & 0.96038 & 0.95981 & 0.95950 & 0.95945 & 0.95971 & 0.95936 \\
& Kappa-like & 0.24936 & 0.22345 & 0.22041 & 0.21179 & 0.21041 & 0.21228 \\
\hline \multirow{2}{*}{10} & GRA & 0.95919 & 0.95963 & 0.95941 & 0.96020 & 0.95935 & 0.95947 \\
& Kappa-like & 0.21885 & 0.21299 & 0.20544 & 0.20376 & 0.21016 & 0.21141 \\
\hline \multirow{2}{*}{20} & GRA & 0.95985 & 0.95982 & 0.95948 & 0.95983 & 0.95947 & 0.95949 \\
& Kappa-like & 0.21380 & 0.20625 & 0.20439 & 0.20729 & 0.210455 & 0.21232 \\
\hline
\end{tabular}

Table 5.1: GRA and Kappa-like averaged values of 1000 replications when the proportion of positive instances is between $8 \%$ and $12 \%$. 


\begin{tabular}{cccccccc}
\hline $\mathrm{m}$ & & $\mathrm{n}=25$ & $\mathrm{n}=50$ & $\mathrm{n}=75$ & $\mathrm{n}=100$ & $\mathrm{n}=500$ & $\mathrm{n}=1000$ \\
\hline \multirow{2}{*}{2} & GRA & 0.98267 & 0.98312 & 0.98250 & 0.98280 & 0.98297 & 0.98300 \\
& Kappa-like & 0.70594 & 0.71279 & 0.71071 & 0.71185 & 0.71468 & 0.71475 \\
\hline \multirow{2}{*}{5} & GRA & 0.98268 & 0.98262 & 0.98303 & 0.98305 & 0.98301 & 0.98299 \\
& Kappa-like & 0.71041 & 0.71185 & 0.71293 & 0.71440 & 0.71474 & 0.71472 \\
\hline \multirow{2}{*}{10} & GRA & 0.98322 & 0.98305 & 0.98304 & 0.98317 & 0.98299 & 0.98302 \\
& Kappa-like & 0.71224 & 0.71364 & 0.71451 & 0.71438 & 0.714706 & 0.71496 \\
\hline \multirow{2}{*}{20} & GRA & 0.98317 & 0.98301 & 0.98307 & 0.98299 & 0.98301 & 0.98307 \\
& Kappa-like & 0.71284 & 0.71375 & 0.71424 & 0.71427 & 0.71481 & 0.71516 \\
\hline
\end{tabular}

Table 5.2: GRA and Kappa-like averaged values of 1000 replications when the proportion of positive instances is between $46 \%$ and $54 \%$.

\subsubsection{GRA versus QICu in terms of the logistic-GEE model complexity}

In this subsection, using simulated data, we compare GRA with QICu in terms of the logistic-GEE model complexity when we are adding covariates one by one into the model.

We generated a data set of binary outcomes containing 5000 clusters with cluster sizes $m$ equal to 10 with exchangeable correlation structure (correlation equal to 0.3 ). To generate the data set we used the model in Eqn. (5.9) with ten covariates. We chose each covariate to be distributed as uniform $(0,1)$ and $\beta_{0}=0, \beta_{1}=10, \beta_{2}=9.5, \beta_{3}=9$, $\beta_{4}=8.5, \beta_{5}=8, \beta_{6}=7.5, \beta_{7}=7, \beta_{8}=6.5, \beta_{9}=6$ and $\beta_{10}=5.5$. Then, we fitted a logistic-GEE model as follows: we started with the intercept-only model and then at each step we added one more covariate into the model (in decreasing order of their magnitudes of regression coefficients) and computed the GRA and QICu (using the R package "MuMIn") of the model. We obtained the relative improvement of each criterion at each step as: the difference (increase for GRA and decrease for QICu) between the value of the criterion in the previous step and the current step, divided by its value in the previous step.

Figures 5.1a and 5.1b present the GRAs and QICus of the model with different numbers of covariates when adding covariates one by one into the model. The QICu and GRA improve (decrease and increase) by increasing the number of covariates in the model as we expected. However, they are very different in terms of their percentages of improvement at each step. We expected that at the early steps (especially at the first step) when we add covariates with larger effects (bigger coefficients) into the model we get higher percentages of goodness-of-fit improvement compared to those covariates we are adding in the last steps. Figures 5.1c and 5.1d illustrate the percentages of improvement in QICu and GRA by adding covariates one by one into the model, respectively. From Figure 5.1d 
we observe that entering the first covariate improves the model's GRA the most, by $49 \%$, whereas the model's QICu improved only by $13 \%$ which is smaller than the percentage of improvement in each of the next eight steps.

The model's GRA reaches 0.97 when there are six covariates in the model and then adding four more covariates improves the GRA with less than $2 \%$, at each step. Contrary to what is expected, percentages of improvement in $\mathrm{QICu}$ are increasing up to the seventh covariate and only the last two covariates improve the model's QICu less than $13 \%$ (less that the first covariate).

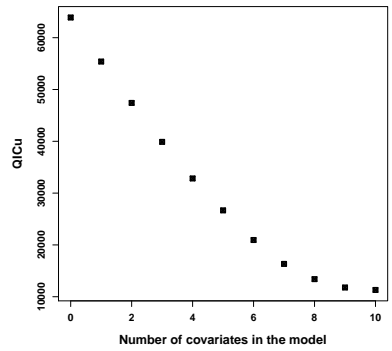

(a)

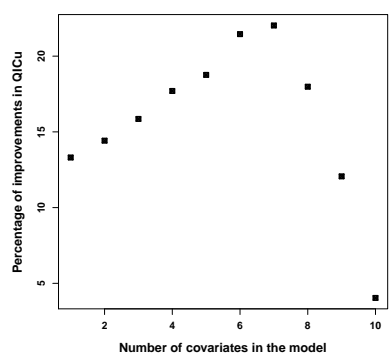

(c)

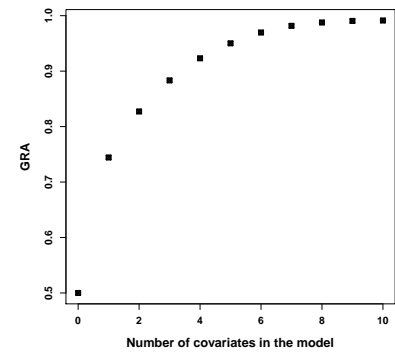

(b)

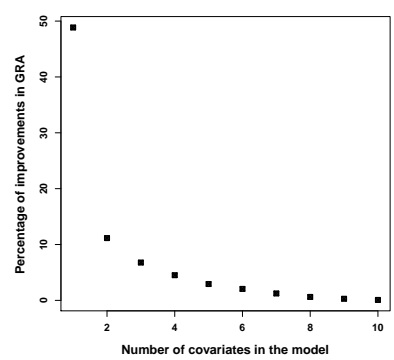

(d)

Figure 5.1: The QICu and GRA as functions of logistic-GEE model complexity.

These results show that unlike GRA, QICu does not exhibit monotonous relative improvements when adding covariates one by one in the logistic-GEE model. For smaller contributions to explaining variation in the outcomes (i.e., for smaller values of the regression coefficients) the relative change in QICu may be larger, but not for GRA. 


\subsubsection{Comparing GRA-CV with QICu as a Model-Selection Criterion}

The selection of the best subset of covariates maximizing the performance of the model is known as the model selection problem. In this subsection we compare GRA-CV (using cluster-out cross-validation) with QICu as the model-selection criterion, when we apply a forward (greedy) model selection process. The forward-selection technique begins with no covariate in the model. As the first step for every covariate we set a univariate model and obtain the GRA-CV and QICu of each model. The first model includes the covariate with the highest model's GRA-CV (the lowest QICu, in the case of using QICu as a selecting criterion). At each following step, each covariate that is not already in the model is tested for inclusion in the model by obtaining its GRA-CV (and QICu) and we then include the covariate that best improves the criterion value.

In the simulation study we consider a number of different logistic-GEE models to compare GRA-CV and QICu as greedy model selection criteria. Table 5.3 presents a list of the models with different parameters that we evaluate. We study the following situations:

(1) the covariates in the models are considerably different in their magnitude of effects (magnitudes of coefficients), models 1-4;

(2) the data is two-class imbalanced data, models 5-8;

(3) the covariates in the models are slightly different in their magnitude of effects, models 9-12;

\begin{tabular}{cccc}
\hline Model & Coefficients & Correlation & Positive instance proportion \\
\hline 1 & $\beta_{1}=1, \beta_{2}=2, \beta_{3}=-3$ & 0.1 & $44 \%$ to $56 \%$ \\
2 & $\beta_{1}=1, \beta_{2}=2, \beta_{3}=-3$ & 0.3 & $44 \%$ to $56 \%$ \\
3 & $\beta_{1}=1, \beta_{2}=2, \beta_{3}=-3$ & 0.6 & $44 \%$ to $56 \%$ \\
4 & $\beta_{1}=1, \beta_{2}=2, \beta_{3}=-3$ & 0.9 & $44 \%$ to $56 \%$ \\
5 & $\beta_{1}=1, \beta_{2}=-2, \beta_{3}=-3$ & 0.1 & $12 \%$ to $20 \%$ \\
6 & $\beta_{1}=1, \beta_{2}=-2, \beta_{3}=-3$ & 0.3 & $12 \%$ to $20 \%$ \\
7 & $\beta_{1}=1, \beta_{2}=-2, \beta_{3}=-3$ & 0.6 & $12 \%$ to $20 \%$ \\
8 & $\beta_{1}=1, \beta_{2}=-2, \beta_{3}=-3$ & 0.9 & $12 \%$ to $20 \%$ \\
9 & $\beta_{1}=1, \beta_{2}=1.3, \beta_{3}=-1.6$ & 0.1 & $52 \%$ to $64 \%$ \\
10 & $\beta_{1}=1, \beta_{2}=1.3, \beta_{3}=-1.6$ & 0.3 & $52 \%$ to $64 \%$ \\
11 & $\beta_{1}=1, \beta_{2}=1.3, \beta_{3}=-1.6$ & 0.6 & $52 \%$ to $64 \%$ \\
12 & $\beta_{1}=1, \beta_{2}=1.3, \beta_{3}=-1.6$ & 0.9 & $52 \%$ to $64 \%$ \\
\hline
\end{tabular}

Table 5.3: Simulation study factors for comparing GRA-CV and QICu as model selection criteria. 


\begin{tabular}{cccccccc}
\hline Model & Criterion & $321^{*}$ & 312 & 231 & 213 & 132 & 123 \\
\hline \multirow{2}{*}{ Model 1 } & QICu & 1000 & 0 & 0 & 0 & 0 & 0 \\
& GRA-CV & 1000 & 0 & 0 & 0 & 0 & 0 \\
\hline \multirow{2}{*}{ Model 2 } & QICu & 1000 & 0 & 0 & 0 & 0 & 0 \\
& GRA-CV & 1000 & 0 & 0 & 0 & 0 & 0 \\
\hline \multirow{2}{*}{ Model 3 } & QICu & 1000 & 0 & 0 & 0 & 0 & 0 \\
& GRA-CV & 1000 & 0 & 0 & 0 & 0 & 0 \\
\hline \multirow{2}{*}{ Model 4 } & QICu & 992 & 5 & 3 & 0 & 0 & 0 \\
& GRA-CV & 996 & 3 & 1 & 0 & 0 & 0 \\
\hline \multirow{2}{*}{ Model 5 } & QICu & 1000 & 0 & 0 & 0 & 0 & 0 \\
& GRA-CV & 1000 & 0 & 0 & 0 & 0 & 0 \\
\hline \multirow{2}{*}{ Model 6 } & QICu & 1000 & 0 & 0 & 0 & 0 & 0 \\
& GRA-CV & 1000 & 0 & 0 & 0 & 0 & 0 \\
\hline \multirow{2}{*}{ Model 7 } & QICu & 994 & 3 & 3 & 0 & 0 & 0 \\
& GRA-CV & 994 & 3 & 3 & 0 & 0 & 0 \\
\hline \multirow{2}{*}{ Model 8 } & QICu & 935 & 31 & 34 & 0 & 0 & 0 \\
& GRA-CV & 941 & 31 & 28 & 0 & 0 & 0 \\
\hline \multirow{2}{*}{ Model 9 } & QICu & 962 & 18 & 20 & 0 & 0 & 0 \\
& GRA-CV & 959 & 21 & 20 & 0 & 0 & 0 \\
\hline \multirow{2}{*}{ Model 10 } & QICu & 907 & 39 & 54 & 0 & 0 & 0 \\
& GRA-CV & 904 & 41 & 55 & 0 & 0 & 0 \\
\hline \multirow{2}{*}{ Model 11 } & QICu & 752 & 120 & 120 & 4 & 3 & 1 \\
& GRA-CV & 758 & 117 & 117 & 4 & 3 & 1 \\
\hline \multirow{2}{*}{ Model 12 } & QICu & 575 & 184 & 193 & 20 & 17 & 11 \\
& GRA-CV & 583 & 183 & 189 & 16 & 20 & 9 \\
\hline
\end{tabular}

Table 5.4: Frequencies of different permutations of covariates $X_{1}, X_{2}, X_{3}$ selected by GRA-CV versus QICu using forward selection method for 1000 replications. The true order is $\left\{X_{3}, X_{2}, X_{1}\right\}$.

*ijk: the set of covariates in the order $\left\{X_{i}, X_{j}, X_{k}\right\}$.

For each model, we generated a data set with $n=500$ clusters with cluster size $m=10$ with exchangeable correlation structure using the model in Eqn. (5.9) where each covariate within a cluster is distributed as uniform $(0,1)$. Then we started two forward selection processes: one using GRA-CV (based on cluster-out cross-validation) and one using QICu as a model selection criterion. We replicated this process 1000 times by generating 1000 independent data sets. Frequencies of the sets of covariates (in different orders) selected by GRA-CV versus QICu for each model are presented in Table 5.4.

Results in Table 5.4 show that both QICu and GRA-CV perform well in selection of the covariates in the right order, when the covariates in the models are considerably different in their magnitude of effects. However, in case of two-class imbalanced data QICu 
and GRA-CV in $93.5 \%$ and $94.1 \%$ of replications, respectively, select the covariates in the right order when the within-cluster correlation equals to 0.9 . That is, even for two-class imbalanced data, both QICu and GRA-CV perform the same when the covariates in the models are considerably different in their magnitude of effects. But when there is a high within-cluster correlation, GRA-CV performs slightly better than QICu. For balanced data, when the covariates in the models are only slightly different in their magnitude of effects and the correlation is 0.1 , both criteria select the covariates in the right order in almost $96 \%$ of replications which decreases to $57.5 \%$ and $58.3 \%$, respectively for QICu and GRA-CV, when correlation increases to 0.9. In general in Table 5.4 we observe that GRA-CV may perform better in some cases when there is a high correlation (equal to 0.6 or 0.9 ) within clusters. We note that GRA-CV uses less data than QICu due to its cross-validation nature, but still has comparable or even better results.

\subsubsection{Comparing GRA with RA}

As mentioned in Section 5.1, RA is not suggested for use as a goodness-of-fit criterion for logistic-GEE models, because the instances from which the model is built are assumed to be correlated (with unknown correlations) within clusters. However, if there are no within-cluster correlations, RA coincides with the Area Under the ROC Curve (AUC) [3] and we may take advantage of having a ROC curve.

In this section we consider different scenarios to assess the magnitude of difference between RA and GRA using simulated data. We assess this difference in terms of different factors, such as: the magnitude of correlation, the number of clusters and cluster sizes, and presence of two-class imbalanced data.

We generated data similar to the procedure described in the previous subsections using the model in Eqn. (5.9) with two covariates and exchangeable correlation structure. For each experiment we generated 1000 data sets and obtained the average and standard deviation (SD) of the RAs and GRAs.

Tables 5.5-5.8 present the averages of RAs and GRAs and their SDs assuming different magnitudes of correlation, numbers of clusters, and cluster sizes. Results in Tables 5.5-5.8 indicate that on average there are negligible differences between GRAs and RAs. We investigated the situations in which there are data sets with a small number of clusters of large sizes, see the results in Table 5.9. The results show that RA may be slightly overoptimistic compared to GRA. We in addition assessed the magnitude of the differences between RA and GRA in the case of two-class imbalanced data; see the results in Table 5.10. The results show that on average GRAs and RAs are almost the same.

In general, the results in Tables 5.5-5.10 indicate that, considering the data sets with different specifications, on average the differences between GRAs and RAs are very small. Therefore, in practice (depending on sensitivity of the analysis) one may opt to 


\begin{tabular}{ccccccc}
\hline $\mathrm{m}$ & & $\mathrm{n}=50$ & $\mathrm{n}=100$ & $\mathrm{n}=200$ & $\mathrm{n}=500$ & $\mathrm{n}=1000$ \\
\hline \multirow{4}{*}{2} & \multirow{2}{*}{ GRA } & 0.943201 & 0.942800 & 0.943364 & 0.943092 & 0.943041 \\
& & $(0.020808)$ & $(0.014367)$ & $(0.009875)$ & $(0.006572)$ & $(0.004476)$ \\
& \multirow{2}{*}{$\mathrm{RA}$} & 0.943201 & 0.942805 & 0.943368 & 0.943093 & 0.943042 \\
& & $(0.020783)$ & $(0.014363)$ & $(0.009875)$ & $(0.006572)$ & $(0.004476)$ \\
\hline \multirow{4}{*}{5} & \multirow{2}{*}{ GRA } & 0.942842 & 0.944597 & 0.943178 & 0.943035 & 0.943204 \\
& & $(0.013212)$ & $(0.010329)$ & $(0.006479)$ & $(0.004070)$ & $(0.002909)$ \\
& \multirow{2}{*}{ RA } & 0.942860 & 0.944619 & 0.943185 & 0.943037 & 0.943205 \\
& & $(0.013200)$ & $(0.010328)$ & $(0.006478)$ & $(0.004069)$ & $(0.002909)$ \\
\hline \multirow{4}{*}{10} & \multirow{2}{*}{ GRA } & 0.943698 & 0.943135 & 0.943330 & 0.943210 & 0.943038 \\
& & $(0.009092)$ & $(0.006513)$ & $(0.004650)$ & $(0.002819)$ & $(0.002060)$ \\
& \multirow{2}{*}{ RA } & 0.943724 & 0.943149 & 0.943330 & 0.943213 & 0.943039 \\
& & $(0.009085)$ & $(0.006509)$ & $(0.004651)$ & $(0.002819)$ & $(0.002060)$ \\
\hline \multirow{4}{*}{20} & \multirow{2}{*}{ GRA } & 0.942931 & 0.943312 & 0.943097 & 0.943220 & 0.943125 \\
& & $(0.006475)$ & $(0.004775)$ & $(0.003274)$ & $(0.002088)$ & $(0.001479)$ \\
& \multirow{2}{*}{ RA } & 0.942958 & 0.943326 & 0.943104 & 0.943213 & 0.943126 \\
& & $(0.006463)$ & $(0.004771)$ & $(0.003273)$ & $(0.002088)$ & $(0.001478)$ \\
\hline
\end{tabular}

Table 5.5: Average and SD (within parentheses) of GRAs and RAs of 1000 replications when $\beta_{0}=0, \beta_{1}=5, \beta_{2}=-5$, correlation equal to 0.1 and the proportion of positive instances is between $46 \%$ to $54 \%$.

\begin{tabular}{ccccccc}
\hline $\mathrm{m}$ & & $\mathrm{n}=50$ & $\mathrm{n}=100$ & $\mathrm{n}=200$ & $\mathrm{n}=500$ & $\mathrm{n}=1000$ \\
\hline \multirow{4}{*}{2} & \multirow{2}{*}{ GRA } & 0.943327 & 0.942997 & 0.9433297 & 0.942989 & 0.942881 \\
& & $(0.021569)$ & $(0.014893)$ & $(0.010358)$ & $(0.006585)$ & $(0.004600)$ \\
& \multirow{2}{*}{$\mathrm{RA}$} & 0.943349 & 0.943020 & 0.943334 & 0.942992 & 0.942883 \\
& & $(0.021532)$ & $(0.014881)$ & $(0.010355)$ & $(0.006584)$ & $(0.004599)$ \\
\hline \multirow{4}{*}{5} & \multirow{2}{*}{ GRA } & 0.943421 & 0.943257 & 0.943051 & 0.943233 & 0.943027 \\
& & $(0.013518)$ & $(0.009688)$ & $(0.006843)$ & $(0.004343)$ & $(0.003107)$ \\
& \multirow{2}{*}{ RA } & 0.943467 & 0.943287 & 0.943064 & 0.943238 & 0.943029 \\
& & $(0.013468)$ & $(0.009672)$ & $(0.006839)$ & $(0.004342)$ & $(0.003106)$ \\
\hline \multirow{4}{*}{10} & \multirow{2}{*}{ GRA } & 0.943039 & 0.943384 & 0.943284 & 0.943165 & 0.943166 \\
& & $(0.010293)$ & $(0.007164)$ & $(0.005059)$ & $(0.003198)$ & $(0.002210)$ \\
& \multirow{2}{*}{ RA } & 0.943102 & 0.943413 & 0.943299 & 0.943171 & 0.943169 \\
& & $(0.010254)$ & $(0.007149)$ & $(0.005054)$ & $(0.003197)$ & $(0.002209)$ \\
\hline \multirow{4}{*}{20} & \multirow{2}{*}{ GRA } & 0.942728 & 0.943074 & 0.943108 & 0.943197 & 0.943083 \\
& & $(0.007995)$ & $(0.005674)$ & $(0.003765)$ & $(0.002540)$ & $(0.001748)$ \\
& \multirow{2}{*}{ RA } & 0.942798 & 0.943105 & 0.943123 & 0.943203 & 0.943086 \\
& & $(0.007942)$ & $(0.005657)$ & $(0.003759)$ & $(0.002538)$ & $(0.001747)$ \\
\hline
\end{tabular}

Table 5.6: Average and SD (within parentheses) of GRAs and RAs of 1000 replications when $\beta_{0}=0, \beta_{1}=5, \beta_{2}=-5$, correlation equal to 0.3 and the proportion of positive instances is between $46 \%$ to $54 \%$. 


\begin{tabular}{ccccccc}
\hline $\mathrm{m}$ & & $\mathrm{n}=50$ & $\mathrm{n}=100$ & $\mathrm{n}=200$ & $\mathrm{n}=500$ & $\mathrm{n}=1000$ \\
\hline \multirow{3}{*}{2} & \multirow{2}{*}{ GRA } & 0.942864 & 0.943243 & 0.943305 & 0.943190 & 0.943053 \\
& & $(0.022794)$ & $(0.015729)$ & $(0.010975)$ & $(0.006960)$ & $(0.004806)$ \\
& \multirow{2}{*}{$\mathrm{RA}$} & 0.942896 & 0.943263 & 0.943316 & 0.943193 & 0.943055 \\
& & $(0.022737)$ & $(0.015712)$ & $(0.010966)$ & $(0.006959)$ & $(0.004805)$ \\
\hline \multirow{3}{*}{5} & \multirow{2}{*}{ GRA } & 0.944019 & 0.943109 & 0.94311 & 0.942940 & 0.943001 \\
& & $(0.015669)$ & $(0.010871)$ & $(0.008049)$ & $(0.004926)$ & $(0.003496)$ \\
& \multirow{2}{*}{ RA } & 0.944095 & 0.943142 & 0.943125 & 0.942947 & 0.943004 \\
& & $(0.015585)$ & $(0.010839)$ & $(0.008038)$ & $(0.004923)$ & $(0.003495)$ \\
\hline \multirow{4}{*}{10} & \multirow{2}{*}{ GRA } & 0.943510 & 0.943314 & 0.942942 & 0.943101 & 0.943222 \\
& & $(0.013632)$ & $(0.009353)$ & $(0.006776)$ & $(0.004212)$ & $(0.002898)$ \\
& \multirow{2}{*}{ RA } & 0.943582 & 0.943351 & 0.942960 & 0.943107 & 0.943226 \\
& & $(0.0135347)$ & $(0.0093223)$ & $(0.006764)$ & $(0.0042096)$ & $(0.0028972)$ \\
\hline \multirow{2}{*}{20} & \multirow{2}{*}{ GRA } & 0.943122 & 0.943419 & 0.943173 & 0.943025 & 0.943243 \\
& & $(0.011472)$ & $(0.008268)$ & $(0.005856)$ & $(0.003810)$ & $(0.002539)$ \\
& \multirow{2}{*}{ RA } & 0.943210 & 0.943456 & 0.943191 & 0.943033 & 0.943246 \\
& & $(0.011371)$ & $(0.008234)$ & $(0.005843)$ & $(0.003807)$ & $(0.002538)$ \\
\hline
\end{tabular}

Table 5.7: Average and SD (within parentheses) of GRAs and RAs of 1000 replications when $\beta_{0}=0, \beta_{1}=5, \beta_{2}=-5$, correlation equal to 0.6 and the proportion of positive instances is between $46 \%$ to $54 \%$.

\begin{tabular}{ccccccc}
\hline $\mathrm{m}$ & & $\mathrm{n}=50$ & $\mathrm{n}=100$ & $\mathrm{n}=200$ & $\mathrm{n}=500$ & $\mathrm{n}=1000$ \\
\hline \multirow{3}{*}{2} & \multirow{2}{*}{ GRA } & 0.943366 & 0.943117 & 0.943263 & 0.943358 & 0.943301 \\
& & $(0.024965)$ & $(0.018496)$ & $(0.012602)$ & $(0.007858)$ & $(0.005429)$ \\
& \multirow{2}{*}{$\mathrm{RA}$} & 0.943396 & 0.943132 & 0.943269 & 0.943360 & 0.943302 \\
& $(0.024903)$ & $(0.018476)$ & $(0.012596)$ & $(0.007856)$ & $(0.005428)$ \\
\hline \multirow{3}{*}{5} & \multirow{2}{*}{ GRA } & 0.942375 & 0.943501 & 0.943490 & 0.943117 & 0.943506 \\
& & $(0.022207)$ & $(0.014922)$ & $(0.010560)$ & $(0.006467)$ & $(0.004491)$ \\
& \multirow{2}{*}{$\mathrm{RA}$} & 0.942432 & 0.943519 & 0.943499 & 0.943121 & 0.943508 \\
& & $(0.022108)$ & $(0.014891)$ & $(0.010549)$ & $(0.006464)$ & $(0.004491)$ \\
\hline \multirow{4}{*}{10} & \multirow{2}{*}{ GRA } & 0.942856 & 0.942854 & 0.943654 & 0.943189 & 0.942946 \\
& & $(0.021065)$ & $(0.013952)$ & $(0.009940)$ & $(0.006384)$ & $(0.004429)$ \\
& \multirow{2}{*}{ RA } & 0.942906 & 0.942878 & 0.943665 & 0.943193 & 0.942948 \\
& & $(0.020963)$ & $(0.013916)$ & $(0.009929)$ & $(0.006382)$ & $(0.004428)$ \\
\hline \multirow{2}{*}{20} & \multirow{2}{*}{ GRA } & 0.942088 & 0.942866 & 0.943188 & 0.9433976 & 0.943319 \\
& & $(0.020537)$ & $(0.014036)$ & $(0.009712)$ & $(0.006272)$ & $(0.004374)$ \\
& \multirow{2}{*}{ RA } & 0.942147 & 0.942893 & 0.943199 & 0.943401 & 0.943321 \\
& & $(0.020423)$ & $(0.013995)$ & $(0.009700)$ & $(0.006268)$ & $(0.004373)$ \\
\hline
\end{tabular}

Table 5.8: Average and SD (within parentheses) of GRAs and RAs of 1000 replications when $\beta_{0}=0, \beta_{1}=5, \beta_{2}=-5$, correlation equal to 0.9 and the proportion of positive instances is between $46 \%$ to $54 \%$. 


\begin{tabular}{cccccc}
\hline $\mathrm{m}$ & & $\mathrm{n}=2$ & $\mathrm{n}=5$ & $\mathrm{n}=10$ & $\mathrm{n}=20$ \\
\hline \multirow{4}{*}{20} & \multirow{2}{*}{ GRA } & 0.939558 & 0.943114 & 0.943596 & 0.942949 \\
& & $(0.049858)$ & $(0.021458)$ & $(0.017361)$ & $(0.012947)$ \\
& \multirow{2}{*}{$\mathrm{RA}$} & 0.944343 & 0.9440347 & 0.943955 & 0.943145 \\
& $(0.037969)$ & $(0.019281)$ & $(0.016848)$ & $(0.012724)$ \\
\hline \multirow{4}{*}{50} & \multirow{2}{*}{ GRA } & 0.941605 & 0.942511 & 0.942575 & 0.943071 \\
& & $(0.039075)$ & $(0.027787)$ & $(0.014080)$ & $(0.009868)$ \\
& \multirow{2}{*}{ RA } & 0.944812 & 0.9436875 & 0.942979 & 0.943241 \\
& & $(0.028668)$ & $(0.025688)$ & $(0.013421)$ & $(0.009651)$ \\
\hline \multirow{4}{*}{100} & \multirow{2}{*}{ GRA } & 0.941950 & 0.942400 & 0.942654 & 0.943342 \\
& & $(0.032201)$ & $(0.018271)$ & $(0.012602)$ & $(0.008886)$ \\
& \multirow{2}{*}{ RA } & 0.945108 & 0.943253 & 0.943059 & 0.943511 \\
& & $(0.022387)$ & $(0.016141)$ & $(0.011890)$ & $(0.008633)$ \\
\hline \multirow{3}{*}{200} & \multirow{2}{*}{ GRA } & 0.942829 & 0.943135 & 0.942741 & 0.943059 \\
& & $(0.031309)$ & $(0.017648)$ & $(0.011756)$ & $(0.007943)$ \\
& \multirow{2}{*}{ RA } & 0.946077 & 0.943960 & 0.943128 & 0.943232 \\
& & $(0.020400)$ & $(0.015379)$ & $(0.011024)$ & $(0.007692)$ \\
\hline
\end{tabular}

Table 5.9: Average and SD (within parentheses) of GRAs and RAs of 1000 replications when $\beta_{0}=0, \beta_{1}=5, \beta_{2}=-5$, correlation equal to 0.3 and the proportion of positive instances is between $46 \%$ to $54 \%$. In these experiments we have relatively few clusters, which however are relatively large.

\begin{tabular}{ccccccc}
\hline $\mathrm{m}$ & & $\mathrm{n}=50$ & $\mathrm{n}=100$ & $\mathrm{n}=200$ & $\mathrm{n}=500$ & $\mathrm{n}=1000$ \\
\hline \multirow{3}{*}{2} & \multirow{2}{*}{ GRA } & 0.909811 & 0.908838 & 0.908888 & 0.908514 & 0.908642 \\
& & $(0.037325)$ & $(0.025555)$ & $(0.017677)$ & $(0.011278)$ & $(0.007962)$ \\
& \multirow{2}{*}{$\mathrm{RA}$} & 0.909665 & 0.908776 & 0.908848 & 0.908501 & 0.908634 \\
& $(0.037336)$ & $(0.025551)$ & $(0.017672)$ & $(0.011277)$ & $(0.007962)$ \\
\hline \multirow{3}{*}{5} & \multirow{2}{*}{ GRA } & 0.909582 & 0.908738 & 0.908176 & 0.908436 & 0.908545 \\
& & $(0.023585)$ & $(0.016895)$ & $(0.011802)$ & $(0.007756)$ & $(0.005533)$ \\
& \multirow{2}{*}{$\mathrm{RA}$} & 0.909374 & 0.908636 & 0.908119 & 0.908415 & 0.908534 \\
& & $(0.023526)$ & $(0.016872)$ & $(0.011796)$ & $(0.007754)$ & $(0.005532)$ \\
\hline \multirow{4}{*}{10} & \multirow{2}{*}{ GRA } & 0.908877 & 0.908615 & 0.908818 & 0.908525 & 0.908371 \\
& & $(0.018811)$ & $(0.012508)$ & $(0.008933)$ & $(0.005672)$ & $(0.004061)$ \\
& \multirow{2}{*}{ RA } & 0.908658 & 0.908484 & 0.908756 & 0.908501 & 0.908358 \\
& & $(0.018736)$ & $(0.012484)$ & $(0.008926)$ & $(0.005670)$ & $(0.004060)$ \\
\hline \multirow{3}{*}{20} & \multirow{2}{*}{ GRA } & 0.908788 & 0.908388 & 0.908255 & 0.908516 & 0.908444 \\
& & $(0.011738)$ & $(0.009589)$ & $(0.006995)$ & $(0.004506)$ & $(0.003080)$ \\
& \multirow{2}{*}{ RA } & 0.908687 & 0.908263 & 0.908191 & 0.908491 & 0.908431 \\
& & $(0.011729)$ & $(0.009565)$ & $(0.006986)$ & $(0.004503)$ & $(0.003079)$ \\
\hline
\end{tabular}

Table 5.10: Average and SD (within parentheses) of GRAs and RAs of 1000 replications when $\beta_{0}=0, \beta_{1}=0.5, \beta_{2}=-5$, correlation equal to 0.3 and the proportion of positive instances is between $8 \%$ to $12 \%$. In these experiments we have unbalanced data. 
neglect this difference and use RA instead of GRA and take advantage of having a ROC curve.

\subsection{Application to the TIME-CHF Study}

In this section, we present an example in order to evaluate GRA-CV as a model-selection criterion using the TIME-CHF data when the method of validation is cluster-out crossvalidation. The design of the TIME-CHF study is described in detail in Section 2.1. The analysis in this section includes 499 patients aged 60 years or older with Hearth Failure (HF), Left Ventricular Ejection Fraction (LVEF) $\leq 45 \%$, New York Heart Association functional classification (NYHA) $\geq$ II.

Six biomarkers are included in this experiment: Prealbumin (PREA), Soluble ST2 (SST2), Interleukin-6 (IL6), High Sensitivity C-Reactive Protein (HSCRP), Growth Differentiation Factor 15 (GDF15), Soluble FMS-Like Tyrosine kinase-1 (SFLT). The biomarkers, systolic Blood Pressure (BPsyst) and Rales were measured at every visit and dosages of the Heart Failure medication Loop (Loop diuretics) were available on a daily basis. In addition to Loop, biomarkers, BPsyst and Rales (1=abnormal lung sounds, $0=$ normal lung sounds) as time dependent covariates we used eight fixed covariates that were measured only at the baseline: Age, Gender ( $1=$ male, $0=$ female), Coronary Artery Disease (CAD), LVEF, Kidney-disease, Diabetes, Anemia, and Charlsonscore (Charlson comorbidity score), where CAD, Kidney-disease, Diabetes, and Anemia are binary variables that indicate whether a patient is suffering from these diseases or not $(1=\mathrm{yes}, 0=\mathrm{no})$.

We defined a binary outcome variable with a value of one if a given patient was hospitalized for HF or died during a given time interval of one month; otherwise the value was zero. The follow-up period was nineteen months. Note that the outcome values for a given patient can change from one time interval to another and are likely to be correlated. Therefore, the logistic-GEE model is a suitable model for this study.

For this analysis, we gave more weight to the outcome death (two times of HF hospitalization) when applying the weighted logistic-GEE model [105]. For patients who either died or withdrew from the study before nineteen months, the number of outcome values equals the number of follow-up months. In order to apply the weighted logistic-GEE model, we also included the covariate values at the same time resolution as the outcomes. Therefore, we converted the covariate values into monthly base variables as follows, see Table 5.11. 


\begin{tabular}{|c|c|c|c|c|c|}
\hline Patient ID & $\begin{array}{c}\text { HF Hospitalization } \\
\text { or death in the next month }\end{array}$ & Time point & Medication & $\begin{array}{c}\text { Biomarkers and } \\
\text { time dependent covariates }\end{array}$ & $\begin{array}{c}\text { Fixed } \\
\text { covariates }\end{array}$ \\
\hline 1 & 0 & $\mathrm{M}_{0}$ & $\mathrm{M}_{0}$ & $\mathrm{M}_{0}$ & $\mathrm{M}_{0}$ \\
\hline 1 & 1 & $\mathrm{M}_{1}$ & $\operatorname{Avg}\left(\mathrm{M}_{0}, \mathrm{M}_{1}\right)$ & $\mathrm{M}_{1}$ & $\mathrm{M}_{0}$ \\
\hline 1 & 0 & $\mathrm{M}_{2}$ & $\operatorname{Avg}\left(M_{1}, M_{2}\right)$ & $\mathrm{M}_{1}$ & $\mathrm{M}_{0}$ \\
\hline 1 & 0 & $\mathrm{M}_{3}$ & $\operatorname{Avg}\left(\mathrm{M}_{2}, \mathrm{M}_{3}\right)$ & $\mathrm{M}_{3}$ & $\mathrm{M}_{0}$ \\
\hline 1 & 1 & $\mathrm{M}_{4}$ & $\operatorname{Avg}\left(M_{3}, M_{4}\right)$ & $\mathrm{M}_{3}$ & $\mathrm{M}_{0}$ \\
\hline 1 & 0 & $\mathrm{M}_{5}$ & $\operatorname{Avg}\left(M_{4}, M_{5}\right)$ & $\mathrm{M}_{3}$ & $\mathrm{M}_{0}$ \\
\hline 1 & 1 & $\mathrm{M}_{6}$ & $\operatorname{Avg}\left(M_{5}, M_{6}\right)$ & $\mathrm{M}_{6}$ & $\mathrm{M}_{0}$ \\
\hline 2 & 1 & $\mathrm{M}_{0}$ & $\mathrm{M}_{0}$ & $\mathrm{M}_{0}$ & $\mathrm{M}_{0}$ \\
\hline 2 & 0 & $\mathrm{M}_{1}$ & $\operatorname{Avg}\left(M_{0}, M_{1}\right)$ & $\mathrm{M}_{1}$ & $\mathrm{M}_{0}$ \\
\hline 2 & 0 & $\mathrm{M}_{2}$ & $\operatorname{Avg}\left(M_{1}, M_{2}\right)$ & $\mathrm{M}_{1}$ & $\mathrm{M}_{0}$ \\
\hline 2 & 1 & $\mathrm{M}_{3}$ & $\operatorname{Avg}\left(M_{2}, M_{3}\right)$ & $\mathrm{M}_{3}$ & $\mathrm{M}_{0}$ \\
\hline 2 & 1 & $\mathrm{M}_{4}$ & $\operatorname{Avg}\left(M_{3}, M_{4}\right)$ & $\mathrm{M}_{3}$ & $\mathrm{M}_{0}$ \\
\hline 2 & 1 & $\mathrm{M}_{5}$ & $\operatorname{Avg}\left(M_{4}, M_{5}\right)$ & $\mathrm{M}_{3}$ & $\mathrm{M}_{0}$ \\
\hline$\vdots$ & $\vdots$ & $\vdots$ & $\vdots$ & $\vdots$ & $\vdots$ \\
\hline 499 & 1 & $\mathrm{M}_{0}$ & $\mathrm{M}_{0}$ & $\mathrm{M}_{0}$ & $\mathrm{M}_{0}$ \\
\hline 499 & 1 & $\mathrm{M}_{1}$ & $\operatorname{Avg}\left(M_{0}, M_{1}\right)$ & $\mathrm{M}_{1}$ & $\mathrm{M}_{0}$ \\
\hline 499 & 0 & $\mathrm{M}_{2}$ & $\operatorname{Avg}\left(M_{1}, M_{2}\right)$ & $\mathrm{M}_{1}$ & $\mathrm{M}_{0}$ \\
\hline 499 & 1 & $\mathrm{M}_{3}$ & $\operatorname{Avg}\left(M_{2}, M_{3}\right)$ & $\mathrm{M}_{3}$ & $\mathrm{M}_{0}$ \\
\hline 499 & 0 & $\mathrm{M}_{4}$ & $\operatorname{Avg}\left(M_{3}, M_{4}\right)$ & $\mathrm{M}_{3}$ & $\mathrm{M}_{0}$ \\
\hline 499 & 0 & $\mathrm{M}_{5}$ & $\operatorname{Avg}\left(M_{4}, M_{5}\right)$ & $\mathrm{M}_{3}$ & $\mathrm{M}_{0}$ \\
\hline 499 & 0 & $\mathrm{M}_{6}$ & $\operatorname{Avg}\left(M_{5}, M_{6}\right)$ & $\mathrm{M}_{6}$ & $\mathrm{M}_{0}$ \\
\hline 499 & 0 & $\mathrm{M}_{7}$ & $\operatorname{Avg}\left(M_{6}, M_{7}\right)$ & $\mathrm{M}_{6}$ & $\mathrm{M}_{0}$ \\
\hline 499 & 0 & $\mathrm{M}_{8}$ & $\operatorname{Avg}\left(M_{7}, M_{8}\right)$ & $\mathrm{M}_{6}$ & $\mathrm{M}_{0}$ \\
\hline 499 & 0 & $\mathrm{M}_{9}$ & $\operatorname{Avg}\left(M_{8}, M_{9}\right)$ & $\mathrm{M}_{6}$ & $\mathrm{M}_{0}$ \\
\hline 499 & 0 & $\mathrm{M}_{10}$ & $\operatorname{Avg}\left(\mathrm{M}_{9}, \mathrm{M}_{10}\right)$ & $\mathrm{M}_{6}$ & $\mathrm{M}_{0}$ \\
\hline 499 & 0 & $\mathrm{M}_{11}$ & $\operatorname{Avg}\left(\mathrm{M}_{10}, \mathrm{M}_{11}\right)$ & $\mathrm{M}_{6}$ & $\mathrm{M}_{0}$ \\
\hline 499 & 0 & $\mathrm{M}_{12}$ & $\operatorname{Avg}\left(\mathrm{M}_{11}, \mathrm{M}_{12}\right)$ & $\mathrm{M}_{12}$ & $\mathrm{M}_{0}$ \\
\hline 499 & 0 & $\mathrm{M}_{13}$ & $\operatorname{Avg}\left(M_{12}, M_{13}\right)$ & $\mathrm{M}_{12}$ & $\mathrm{M}_{0}$ \\
\hline 499 & 0 & $\mathrm{M}_{14}$ & $\operatorname{Avg}\left(\mathrm{M}_{13}, \mathrm{M}_{14}\right)$ & $\mathrm{M}_{12}$ & $\mathrm{M}_{0}$ \\
\hline 499 & 0 & $\mathrm{M}_{15}$ & $\operatorname{Avg}\left(M_{14}, M_{15}\right)$ & $\mathrm{M}_{12}$ & $\mathrm{M}_{0}$ \\
\hline 499 & 0 & $\mathrm{M}_{16}$ & $\operatorname{Avg}\left(M_{15}, M_{16}\right)$ & $\mathrm{M}_{12}$ & $\mathrm{M}_{0}$ \\
\hline 499 & 0 & $\mathrm{M}_{17}$ & $\operatorname{Avg}\left(\mathrm{M}_{16}, \mathrm{M}_{17}\right)$ & $\mathrm{M}_{12}$ & $\mathrm{M}_{0}$ \\
\hline 499 & 0 & $\mathrm{M}_{18}$ & $\operatorname{Avg}\left(\mathrm{M}_{17}, \mathrm{M}_{18}\right)$ & $\mathrm{M}_{18}$ & $\mathrm{M}_{0}$ \\
\hline
\end{tabular}

Table 5.11: Layout of dataset for applying logistic-GEE models. $\mathrm{M}_{\mathrm{i}}$ : month $i ; \mathrm{M}_{0}$ : baseline; Avg: average. 


\begin{tabular}{ccccc}
\hline Step & GRA-CV-Covariates $^{\dagger}$ & GRA-CV & QICu & QICu-Covariates $^{\ddagger}$ \\
\hline 0 & No covariate & 0.5 & 1917.965 & No covariate \\
\hline 1 & GDF15 & 0.738816 & 1753.5338 & GDF15 \\
\hline 2 & SST2 & 0.767409 & 1691.7556 & SST2 \\
\hline 3 & CAD & 0.775987 & 1660.4315 & Loop \\
\hline 4 & Loop & 0.784891 & 1649.2790 & CAD \\
\hline 5 & HSCRP & 0.790796 & 1645.5022 & BPsyst \\
\hline 6 & Age & 0.794360 & 1639.8644 & Age \\
\hline 7 & BPsyst & 0.795732 & 1635.7392 & HSCRP \\
\hline 8 & Rales & 0.796831 & 1635.2799 & Rales \\
\hline 9 & Gender & 0.796649 & 1636.1769 & SFLT \\
\hline 10 & SFLT & 0.796255 & 1636.2276 & Anemia \\
\hline 11 & Diabetes & 0.795786 & 1638.1868 & Gender \\
\hline 12 & LVEF & 0.794863 & 1640.6347 & IL6 \\
\hline 13 & PREA & 0.793678 & 1643.1331 & PREA \\
\hline 14 & Charlsonscore & 0.792385 & 1645.8925 & Diabetes \\
\hline 15 & IL6 & 0.791043 & 1648.6419 & LVEF \\
\hline 16 & Kidney-disease & 0.789549 & 1651.4346 & Kidney-disease \\
\hline 17 & Anemia & 0.787360 & 1654.2852 & Charlsonscore \\
\hline
\end{tabular}

Table 5.12: Selected covariates at each step of forward model-selection method.

$\dagger$ Selected covariates at each step using GRA-CV (with one-cluster-out cross-validation) as a model-selection criterion.

$¥$ Selected covariates at each step using QICu as a model-selection criterion.

The bolded covariates are those that are selected.

The medication covariate Loop was down-sampled to monthly values by taking the average drug dosage during the previous month. As the first observation of drug dosage, in the absence of a previous month, the dosage at baseline was used. The biomarkers, BPsyst and Rales were measured at the scheduled follow-up visits (baseline, $1^{\text {st }}, 3^{\text {rd }}, 6^{\text {th }}$, $12^{\text {th }}$ and $18^{\text {th }}$ months). To get the monthly values between these six visits, we used the last observation carried forward method (LOCF) [144] and put the value of the previous visit. For other fixed covariates we used the baseline value at every time interval. The data layout is further illustrated in Table 5.11.

In this setup we applied the forward model-selection method, using either only GRA$\mathrm{CV}$ or only $\mathrm{QICu}$ as a model-selection criterion to find the best subset of covariates through a weighted logistic-GEE model. As explained in Section 5.3.4 the forward modelselection method begins with no covariate in the model. At each step, each covariate is 
tested for inclusion in the model by obtaining the GRA-CVs (and QICus) and we included the covariate that best increased (decreased) GRA-CV $(\mathrm{QICu})$.

Table 5.12 shows the selected covariates for weighted logistic-GEE at each step, separately when, we apply GRA-CV and QICu as the model-selection criteria. We observe from Table 5.12 that GRA-CV increases in the first eight steps, QICu decreases, and after that no covariate inclusion further improves GRA-CV (and QICu). Using both criteria leads to the same selected subset of covariates, (GDF15, SST2, CAD, Loop, HSCRP, Age, BPsyst and Rales), however, the selected subsets were obtained in slightly different orders for each criterion.

\subsection{Conclusion}

In this chapter we proposed GRA as a new measure for assessing the goodness-of-fit as well as the predictive performance of logistic-GEE models. GRA was defined as the probability that a randomly selected instance with positive outcome is ranked higher than a randomly selected instance with negative outcome from another cluster. There are several advantages with GRA compared with other standard goodness-of-fit criteria for logisticGEE models: (a) there is no restriction in the computation of GRA regarding the number and types of covariates in the model, unlike the goodness-of-fit test and statistic proposed by Barnhart and Williamson [13] and Pan [98]; (b) in Section 5.3.2 a simulation study showed that GRA is not biased in the presence of two-class imbalanced data, whereas by contrast the Kappa-like statistic tends to indicate a poor fit of the model; (c) GRA is a probability that is fairly easy to interpret, unlike the Kappa-like statistic and QICu; (d) in contrast with QICu as a widely used goodness-of-fit and model-selection criterion for GEE models, GRA has an absolute range and can therefore be used for comparing the goodness-of-fit of different logistic-GEE models for different data sets; (e) by using crossvalidation, GRA-CV can be used as a predictive performance criterion for logistic-GEE models.

In a simulation study, we also compared the behavior of GRA with QICu as a function of logistic-GEE model complexity. The results showed that unlike GRA, QICu does not perfectly reflect model improvements when adding covariates one by one into the logisticGEE model. As a model-selection criterion, both QICu and GRA-CV (with cluster-out cross-validation) performed well in selecting of the covariates in the right order, when the covariates are considerably different in their magnitude of effects, even in case of twoclass imbalanced data. In models for which the covariates are only slightly different in their magnitude of effects, GRA-CV performed better than QICu when the within-cluster correlation was high (equal to 0.6 and 0.9). In general GRA-CV had comparable results or even better results than $\mathrm{QICu}$, although GRA-CV uses cross-validation, which reduces possibilities for overfitting. 
The simulation study also showed that considering effects of the magnitude of correlation, the number of clusters, size of clusters, and the proportion of positive instances, on average the obtained GRAs and RAs are almost the same. Therefore, in practice researchers may opt to use RA instead of GRA and take advantage of having a ROC curve and using available software packages for calculating the RA.

Note that, in this chapter, in our simulation study we only studied limited values for the parameters and other factors to assess the performances of GRA and GRA-CV. This implies that our findings are to be regarded as preliminary, but promising. It therefore could be interesting to extend the simulation study considering other values for the size of the data, cluster sizes, magnitude and type of within-cluster correlation, regression coefficients and using different type of cross-validation to evaluate GRA and GRA-CV more precisely.

Finally, we note that although GRA has been initially designed for logistic-GEE models, it is applicable to any model for bipartite ranking trained on clustered data. This is due to the fact that GRA employs the model's probabilities and data labels; i.e., it only uses a score but it does not use any internal information from the model being tested. Thus, we conclude that GRA is applicable as a general measure for models for bipartite ranking trained on clustered data. 


\section{Novel Concept to Guide Systolic Heart Failure Medication by Repeated Biomarker Testing}

This chapter is based on: Davarzani, N., Sanders-van Wijk S., Maeder M.T., Rickenbacher P., Smirnov E., Karel J., Suter T., de Boer R.A., Block D., Rolny V., Zaugg Ch., Pfisterer M.E., Peeters, R., Brunner-La Rocca, H.P. Novel concept to guide systolic heart failure medication by repeated biomarker testing - results from TIME-CHF in context of predictive, preventive and personalised medicine. The EPMA Journal, 2018. To appear. 


\begin{abstract}
Background: It is uncertain whether repeated measurements of biomarkers may help to optimize medical HF therapy in order to improve outcome in chronic heart failure (HF).

Methods and Results: This analysis included 499 patients from the Trial of Intensified versus Standard Medical Therapy in Elderly Patients with Congestive Heart Failure (TIME-CHF), aged $\geq 60$ years, $\mathrm{LVEF} \leq 45 \%$, NYHA $\geq$ II who had repeated clinical visits within 19 months follow-up. The interaction between repeated measurements of biomarkers and treatment effects of loop diuretics, spironolactone, $\beta$-blockers and ReninAngiotensin-System (RAS) blockers on risk of HF hospitalization or death was investigated, in a hypothesis generating analysis. Generalized Estimating Equations (GEE) methods were used to account for the correlation between recurrences of events in a patient.
\end{abstract}

Results: One hundred patients (20\%) had just one event (HF hospitalization or death) and $87(17.4 \%)$ had at least two events. Loop diuretics up-titration had a beneficial effect for patients with high Interleukin-6 (IL6) or high High-Sensitivity C-Reactive Protein (HSCRP) (interaction, $\mathrm{P}=0.013$ and $\mathrm{P}=0.001$ ), whereas the opposite was the case with low HSCRP (interaction, $\mathrm{P}=0.013$ ). Higher dosage of loop diuretics was associated with poor outcome in patients with high Blood Urea Nitrogen (BUN) or prealbumin (interaction, $\mathrm{P}=0.006$ and $\mathrm{P}=0.001$ ), but not in those with low levels of these biomarkers. Spironolactone up-titration was associated with lower risk of HF hospitalization or death in patients with high Cystatin $\mathrm{C}(\mathrm{Cys} \mathrm{C})$ (interaction, $\mathrm{P}=0.021)$. $\beta$-blockers up-titration might have a beneficial effect in patients with low Soluble Fms-Like Tyrosine kinase-1 SFLT (interaction, $\mathrm{P}=0.021$ ). No treatment biomarker interactions were found for RAS blockers.

Conclusion: The data of this post hoc analysis suggest that repeated measurements of biomarkers might be used to tailor HF treatment individually. Clearly, prospective testing is needed before this novel concept can be adopted. 
82

Chapter 6. Novel Concept to Guide Systolic Heart Failure Medication by Repeated Biomarker

\subsection{Introduction}

Medical therapy for chronic heart failure (HF) with reduced Ejection Fraction (HFrEF) has evolved greatly over the past decades [88]. Several medication classes - i.e. ReninAngiotensin System (RAS) blockers encompassing Angiotensin-Converting-Enzyme (ACE)inhibitors and Angiotensin-Receptor Blockers (ARBs), $\beta$-blockers and Mineralocorticoid Receptor Antagonists (MRAs) - have been shown to improve prognosis in HFrEF and are therefore recommended in high doses by current guidelines [103, 145]. In addition, (loop) diuretics are recommended for decongestion to relieve symptoms [103, 145]. However, in clinical practice, it is often difficult to implement all medication classes and especially to reach guideline recommended dosages, especially in elderly and comorbid patients [78].

N-terminal pro-B-type Natriuretic Peptide (NT-proBNP) is widely used and accepted as diagnostic and prognostic biomarker in HF [133]. The level of NT-proBNP is changing as response to therapy $[60,125]$. Therefore, it was suggested as a tool to tailor and intensify medical HF therapy.

Several trials and meta-analyses suggest that performing repeated measurements of (NT-pro)BNP may help to establish guideline recommended medical therapy in HFrEF patients to improve outcome [65, 96]. However, the large GUIDE-IT trial [35] that aimed to prove this concept in a sufficiently large patient population was stopped early due to futility [1]. Moreover, guiding HF therapy by a single marker is limited because one biomarker cannot cover the extensive pathophysiological pathways involved in HF. In fact, the approach to guide therapy using (NT-pro)BNP is based on the idea that patients at highest risk of poor outcome are in need of intensified therapy, but there is no specific tailoring of individual drugs.

Since different medication classes in HF interfere with different pathways, a combination of biomarkers that reflect these pathways may be better suited to indicate which medication class is most important to up or possibly down-titrate in a specific patient. That may lead towards precision medicine in HF. Such an attempt has, however, never been made.

As a first step towards the development of a biomarker-guided treatment algorithm for individually tailoring medical HFrEF therapy, we, therefore, investigated the interaction between multiple repeatedly measured biomarkers and the response to the four most important classes of HF medication regarding the risk of HF hospitalization or death. Thus, the main objective of the study, as a purely hypothesis generating study, was to explore which biomarkers in repeated testing would be most predictive of the response to HF drugs during follow-up. 


\subsection{Methods}

\subsubsection{Study and Design}

Since an important prerequisite to address the objective was the availability of detailed data on patient characteristics at different time points, medication over time, and repeatedly measured multiple biomarkers, we used the database of the Trial of Intensified vs. standard Medical therapy in Elderly patients congestive heart failure (TIME-CHF) for this analysis. The study design and methods of the TIME-CHF have been described in detail in Chapter 1. In brief, the study included 499 patients aged 60 years or older with symptomatic HF, Left Ventricular Ejection Fraction (LVEF) $\leq 45 \%$, New York Heart Association functional classification (NYHA) $\geq$ II, from 15 centers in Switzerland and Germany.

After baseline assessment, patients were followed for 5 pre-specified compulsory visits after 1, 3, 6, 12, and 18 months. For each patient, time to recurrence of clinical events was recorded, up to 5.5 years. The primary endpoint for the present analysis was the combined endpoint of HF hospitalization or death during the 18 months trial period plus one month of additional follow-up, i.e. 19 months in total.

History was taken, patients were clinically investigated and blood samples were drawn at every visit. Samples were stored at $-80^{\circ} \mathrm{C}$ until analysis. At the end of the trial, twenty biomarkers were measured from these stored samples at all available visits. Selection of biomarkers was based on the representation of different pathways that are known to reflect important pathophysiological pathways as previously reported [120]. Daily medication doses for all drugs including the four drug classes investigated in this analysis, i.e. $\beta$-blockers, RAS blockers, spironolactone, and loop diuretics were available daily as described [112].

The study was approved by the ethics committees of each center and each patient gave written informed consent before entering the study.

\subsubsection{Data Description}

The study contains three types of covariates presented in Table 6.1:

Patient characteristics: Age, gender, coronary artery disease (CAD), Charlson co-morbidity score, LVEF and history of kidney disease were recorded only at the baseline visit. Systolic blood pressure (BPsyst) and rales on auscultation were recorded at every visit. These 8 characteristics were used as covariates in the multivariable model in this chapter.

Biomarkers: The biomarkers in the TIME-CHF study were measured at every visit as 
84

Chapter 6. Novel Concept to Guide Systolic Heart Failure Medication by Repeated Biomarker Testing

we explained in Section 2.1. Table 2.2 illustrates all the biomarkers that were used in this study.

HF medications: The four most important classes of HF medications were considered for this analysis, i.e. $\beta$-blockers, RAS blockers, spironolactone, and loop diuretics were recorded on a daily basis for each patient, as explained in Section 2.1.

Outcome measurements: For the present analysis any HF hospitalization or death occurring at each month during the nineteen months follow-up were considered as outcome events (primary endpoint).

\subsubsection{Statistical methods}

Patient characteristics, biomarkers at baseline and average medication dosages are presented as mean and standard deviation (SD) for continuous normally distributed variables, median and interquartile range for non-normally distributed continuous variables, or as numbers and percentages for categorical variables (Table 6.1). Variables were compared between those patients without an event and those who experienced an event (i.e. HF hospitalization or death) within nineteen months follow-up. Differences in these variables per number of events (none vs. at least one) were assessed using a t-test for continuous normally distributed variables, a Mann-Whitney U-test for non-normally distributed continuous variables, and a $\chi^{2}$-test for categorical variables. All tests were two-sided at a 5 percent level of significance. Calculations were performed with the use of the SPSS statistical package version 22.0.

The main objective of the study was to explore which biomarkers are most predictive for the response to HF drugs during the follow-up period. In order to achieve this, we tested whether there was a significant interaction between biomarkers and further treatment effects of the four medication classes in our cohort of HF patients, applying the weighted logistic Generalized Estimating Equations (logistic-GEE) model [47, 147, 148]. Logistic-GEE model were applied using R (version 3.3.2, package 'geepack').

To this end, we defined a binary outcome variable with a value of one if a given patient was hospitalized for HF or died during a certain time interval of follow up; otherwise the value was zero. Note that the outcome values for a given patient can change from one time interval to another and that these outcome values are likely to be correlated. For this analysis, we discretized using time intervals of 1 month and gave more weight to the outcome death (two times that of HF hospitalization) when applying the weighted logistic-GEE model [105]. Giving a weight of three to the outcome death resulted in the same findings as for a weight of two. For patients who either died or withdrew from the study before nineteen months, the number of outcome values equals the number of follow 
up months. In order to apply the weighted logistic-GEE model, we also included the covariate values at the same time resolution as the outcomes. Therefore, we converted the covariate values into monthly base variables as explained in Section 5.4, see Table 5.11.

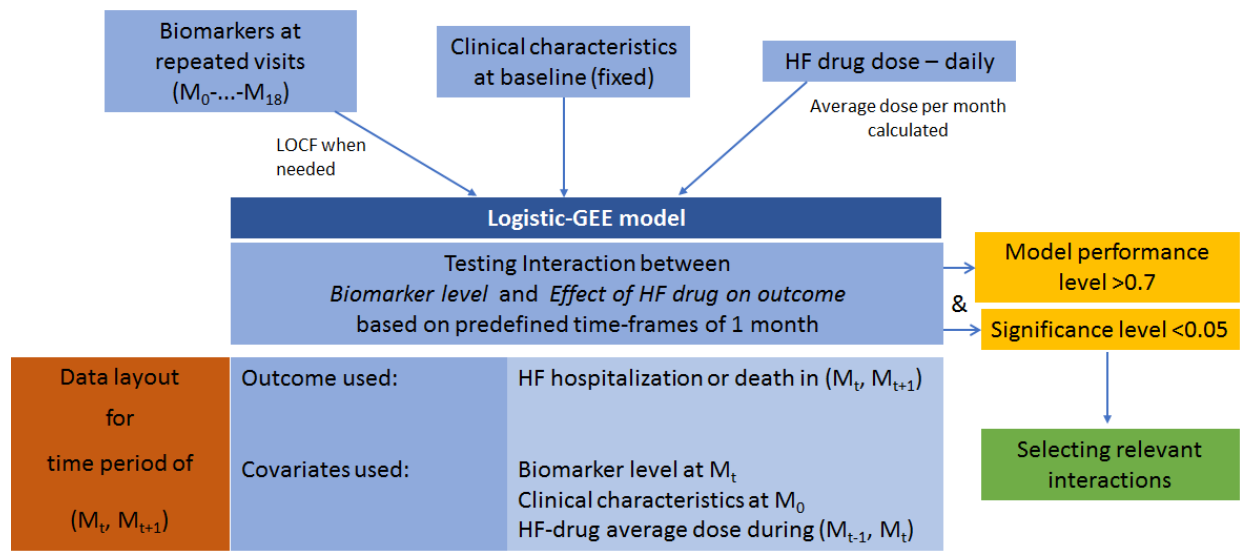

Figure 6.1: Method layout for assessing the interactions between biomarkers and medications. $\mathrm{M}_{\mathrm{t}}$ : month $\mathrm{t} ; \mathrm{M}_{0}$ : baseline.

LOCF: last observation carried forward method.

In order to test the interaction between a given biomarker (in logarithmic form) and medication in the presence of other patient characteristics, we use the following weighted logistic-GEE model:

$$
\begin{aligned}
\operatorname{logit}\left(\mathrm{P}_{\mathrm{it}}\right) & =b_{0}+b_{1} \text { Age }_{\mathrm{it}}+b_{2} \text { Gender }_{\mathrm{it}}+b_{3} \mathrm{CAD}_{\mathrm{it}}+b_{4} \text { CharlsonScore }_{\mathrm{it}}+b_{5} \mathrm{LVEF}_{\mathrm{it}} \\
& +b_{6} \text { KidneyDisease }_{\mathrm{it}}+b_{7} \text { Rales }_{\mathrm{it}}+b_{8} \text { BPsyst }_{\mathrm{it}}+b_{9} \text { Biomarker }_{\mathrm{it}}+b_{10} \text { Medication }_{\mathrm{it}} \\
& +\beta \text { Biomarker }_{\mathrm{it}} \times \text { Medication }_{\mathrm{it}}, i=1, \ldots, 499, t=0, \ldots, 18
\end{aligned}
$$

where, $P_{i t}$ is the probability of hospitalization or death in the month following month $t$ for patient $i$ and Covariate $_{\text {it }}$ is the value of the covariate at month $t$ for patient $i$. Biomarker $\times$ Medication indicates the interaction term and $\beta$ is the interaction coefficient.

In order to investigate whether medications have a different effect on risk of HF hospitalization or death for certain levels of the biomarkers, the interaction coefficient $\beta$ was tested for all possible paired combinations of medication classes and biomarkers. We used GRA-CV using one-cluster-out cross-validation, see Chapter 5, to estimate the predictive performance of the models. In this study, only those logistic-GEE models with a good predictive performance - set at a GRA-CV of $>0.7$ - were predefined as being statistically solid and meaningful (see Figure 6.1). 
Chapter 6. Novel Concept to Guide Systolic Heart Failure Medication by Repeated Biomarker 86

Testing

\subsection{Results}

The frequency of HF hospitalizations (including death) during the nineteen months followup is presented in Figure 6.2. Of the 499 patients, 312 (62.5\%) did not experience any HF hospitalization and were alive after nineteen months, 100 (20\%) had one and $87(17.4 \%)$ had at least two HF related events (HF hospitalization or death) within the nineteen month follow-up period.

Concentrations of biomarkers, baseline characteristics and average drug dosages during the first month are shown in Table 6.1. In comparison to patients without event, those with event(s) were older and more likely to have coronary artery disease, kidney disease, rales, more comorbidities and higher NYHA-class. Moreover, they had higher SFLT, GDF15, CYSC, IL6, STFR, HSTNT, Uric acid, BUN, SST2, NT-proBNP, Creatinine and HSCRP at the baseline and higher average dosages of loop diuretic and spironolactone during the first month, whereas RAS blockers dose was lower.

The $\mathrm{p}$-values of testing interaction (Biomarker $\times$ Medication) in 84 weighted logisticGEE models (covering all possible paired combinations of 4 medications and 20 biomarkers and PLGF/sFlt) and their corresponding GRA-CVs, are presented in Table 6.2.

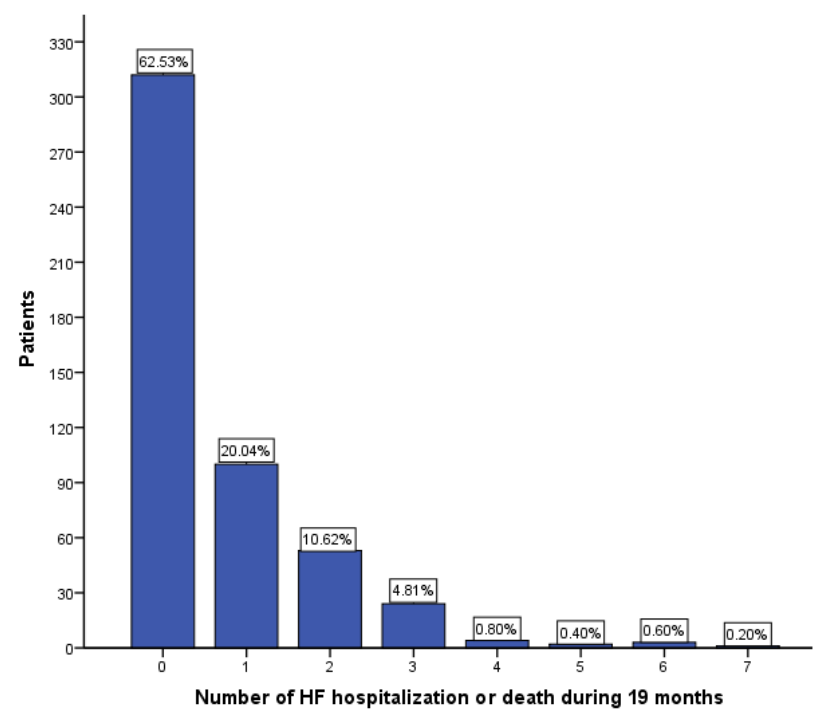

Figure 6.2: The frequency of HF hospitalizations (including death) during 19 months follow up. 


\begin{tabular}{|c|c|c|c|c|}
\hline & All Patients & No events & One or more events & ${ }^{\dagger} \mathrm{p}$-value \\
\hline Baseline characteristics & $(n=499)$ & $(\mathrm{n}=312)$ & $(\mathrm{n}=187)$ & \\
\hline Age (years), mean(sd) & $76.1(7.5)$ & $75.1(7.5)$ & $77.9(7.2)$ & 0.000 \\
\hline Male gender $(\%)$ & $327(65.5)$ & $200(64.1)$ & $127(67.9)$ & 0.386 \\
\hline $\mathrm{CAD}(\%)$ & $287(57.5)$ & $153(49)$ & 134 (71.7) & 0.000 \\
\hline Charlson Score, median [IQR] & $3[2-4]$ & $3[2-4]$ & $3[2-5]$ & 0.000 \\
\hline $\operatorname{LVEF}(\%)(\mathrm{sd})$ & $29.8(7.8)$ & $29.7(7.7)$ & $29.9(8.0)$ & 0.844 \\
\hline Kidney disease (\%) & $277(55.5)$ & $150(48.1)$ & $127(67.9)$ & 0.000 \\
\hline BPsyst, mean (sd) & $118.5(18)$ & $119.6(18)$ & $116.7(18.1)$ & 0.098 \\
\hline Rales $(\%)$ & $209(42.1)$ & $100(35.5)$ & $99(52.9)$ & 0.000 \\
\hline NYHA > II (\%) & $371(74.3)$ & $219(70.2)$ & $152(81.3)$ & 0.006 \\
\hline \multicolumn{5}{|l|}{ Biomarkers, median [IQR] } \\
\hline SFLT & $98.8[81-128]$ & $93.9[77.3-124.2]$ & $105.6[88.4-132.8]$ & 0.000 \\
\hline GDF15 & $3940[2697-5891]$ & 3530 [2416-5125] & 4786 [3433-7183] & 0.000 \\
\hline CYSC & $1.7[1.4-2.1]$ & $1.6[1.3-1.9]$ & $1.9[1.6-2.4]$ & 0.000 \\
\hline Ferritin & 152 [80-258] & 159 [85-261] & $151[66-248]$ & 0.314 \\
\hline IL6 & $7.3[3.9-14.1]$ & $6.6[3.5-11.9]$ & 9.3 [4.6-16.8] & 0.001 \\
\hline PLGF & $22.6[18.3-26.5]$ & $22.3[18.0-26.1]$ & $22.8[18.8-27.1]$ & 0.222 \\
\hline SHBG & $30.1[22.3-40.6]$ & $30.1[22.7-42.3]$ & 30.1 [21.4-38.9] & 0.232 \\
\hline STFR & $4.1[3.2-5.4]$ & $3.9[3.0-5.2]$ & 4.3 [3.3-5.9] & 0.016 \\
\hline HSTNT & 33.6 [19.1-62.7] & 28.6 [17.9-53.3] & $45.8[24.4-85.4]$ & 0.000 \\
\hline TP1NP & 36.7 [23.7-55.5] & 34.9 [23.7-51.5] & 38.2 [23.7-62] & 0.170 \\
\hline Uric & $7.7[6.1-9.2]$ & $7.3[5.9-8.8]$ & $8[6.7-9.5]$ & 0.003 \\
\hline BUN & 10.4 [7.6-13.5] & 9.4 [7.3-12] & $12.5[8.6-16.1]$ & 0.000 \\
\hline SST2 & 35.9 [26-54] & 32.5 [24-45.5] & 43.5 [31-64.2] & 0.000 \\
\hline NT-proBNP & 4194 [2270-7414] & 3675 [1831- 6301] & 5465 [3049-9743] & 0.000 \\
\hline Creatinine & 109 [88-141] & 102 [84-127] & 132 [99-157] & 0.000 \\
\hline HSCRP & 6.7 [2.5-15.8] & $5.5[1.9-14.8]$ & $8.9[3.6-20.4]$ & 0.008 \\
\hline PREA & $0.19[0.15-0.23]$ & $2.00[0.15-0.24]$ & 0.17 [0.14-0.22] & 0.015 \\
\hline OPN & $26.0[17.0-40.9]$ & $22.6[16.1-33.6]$ & $33.5[21.2-55.2]$ & 0.000 \\
\hline Mimican & 116 [85.9-164] & 107 [84.1-145] & $143[93.4-198]$ & 0.000 \\
\hline IGFBP7 & 242 [201-291] & 226 [197-274] & 265 [221-315] & 0.000 \\
\hline \multicolumn{5}{|l|}{ Medications, median [IQR] } \\
\hline RAS blockers & $59.7[44.3-100]$ & $59.6[44.3-100]$ & $50[40.3-100]$ & 0.048 \\
\hline$\beta$-blockers & $25[11.7-50]$ & $25[12.1-50]$ & $25[10.5-46.1]$ & 0.119 \\
\hline Loop diuretic & $60.6[40-92.4]$ & $43.2[33.1-80]$ & 80 [40-129] & 0.000 \\
\hline Spironolactone & $1.6[0-25]$ & $0[0-25]$ & $12.5[0-25]$ & 0.003 \\
\hline
\end{tabular}

Table 6.1: Baseline characteristics and biomarkers and the average drug dosages at the first month in patients without versus with event (HF hospitalizations or death) within nineteen months.

${ }^{\dagger} \mathrm{p}$-value of testing whether the variables are the same in the mean (for continuous normally distributed variables) or median (for continuous non-normally distributed variables) or percentage (for categorical variables) between those patients not hospitalized and those hospitalized or died (two sided t-test or Mann-Whitney U-test for continuous variables and $\chi^{2}$ test for categorical variables). IQR: interquartile range. 


\begin{tabular}{|c|c|c|c|c|c|}
\hline Biomarkers & & $\beta$-blockers & LOOP diuretics & Spironolactone & RAS blockers \\
\hline \multirow{2}{*}{ SFLT } & p-value & 0.02 & 0.33 & 0.28 & 0.50 \\
\hline & GRA-CV & 0.74 & 0.76 & 0.73 & 0.74 \\
\hline \multirow{2}{*}{ GDF15 } & p-value & 0.57 & 0.45 & 0.35 & 0.13 \\
\hline & GRA-CV & 0.75 & 0.76 & 0.75 & 0.75 \\
\hline \multirow{2}{*}{ CYSC } & p-value & 0.83 & 0.57 & 0.02 & 0.40 \\
\hline & GRA-CV & 0.70 & 0.72 & 0.70 & 0.71 \\
\hline \multirow{2}{*}{ Ferritin } & p-value & 0.50 & 0.91 & 0.31 & 0.47 \\
\hline & GRA-CV & 0.66 & 0.70 & 0.66 & 0.67 \\
\hline \multirow{2}{*}{ IL6 } & p-value & 0.27 & 0.01 & 0.43 & 0.43 \\
\hline & GRA-CV & 0.73 & 0.75 & 0.73 & 0.73 \\
\hline \multirow{2}{*}{ PLGF } & p-value & 0.20 & 0.52 & 0.04 & 0.33 \\
\hline & GRA-CV & 0.67 & 0.71 & 0.66 & 0.67 \\
\hline \multirow{2}{*}{ SHBG } & p-value & 0.58 & 0.86 & 0.47 & 0.12 \\
\hline & GRA-CV & 0.65 & 0.69 & 0.65 & 0.67 \\
\hline \multirow{2}{*}{ STFR } & p-value & 0.09 & 0.19 & 0.90 & 0.88 \\
\hline & GRA-CV & 0.71 & 0.73 & 0.70 & 0.71 \\
\hline \multirow{2}{*}{ HSTNT } & p-value & 0.42 & 0.52 & 0.44 & 0.95 \\
\hline & GRA-CV & 0.70 & 0.73 & 0.70 & 0.71 \\
\hline \multirow{2}{*}{ TP1NP } & p-value & 0.04 & 0.52 & 0.54 & 0.28 \\
\hline & GRA-CV & 0.67 & 0.71 & 0.66 & 0.67 \\
\hline \multirow{2}{*}{ Uric } & p-value & 0.18 & 0.48 & 0.46 & 0.27 \\
\hline & GRA-CV & 0.67 & 0.70 & 0.66 & 0.68 \\
\hline \multirow{2}{*}{ BUN } & p-value & 0.86 & 0.00 & 0.26 & 0.55 \\
\hline & GRA-CV & 0.67 & 0.70 & 0.67 & 0.69 \\
\hline \multirow{2}{*}{ SST2 } & p-value & 0.53 & 0.27 & 0.63 & 0.41 \\
\hline & GRA-CV & 0.76 & 0.77 & 0.76 & 0.77 \\
\hline \multirow{2}{*}{ NT-proBNP } & $\mathrm{p}$-value & 0.81 & 0.77 & 0.89 & 0.06 \\
\hline & GRA-CV & 0.75 & 0.76 & 0.74 & 0.76 \\
\hline \multirow{2}{*}{ Creatinine } & $\mathrm{p}$-value & 0.82 & 0.07 & 0.12 & 0.58 \\
\hline & GRA-CV & 0.69 & 0.71 & 0.68 & 0.70 \\
\hline \multirow{2}{*}{ HSCRP } & $\mathrm{p}$-value & 0.26 & 0.00 & 0.21 & 0.40 \\
\hline & GRA-CV & 0.72 & 0.75 & 0.71 & 0.72 \\
\hline \multirow{2}{*}{ PREA } & $\mathrm{p}$-value & 0.29 & 0.00 & 0.90 & 0.88 \\
\hline & GRA-CV & 0.69 & 0.74 & 0.69 & 0.70 \\
\hline \multirow{2}{*}{ OPN } & $\mathrm{p}$-value & 0.30 & 0.96 & 0.09 & 0.79 \\
\hline & GRA-CV & 0.70 & 0.72 & 0.70 & 0.71 \\
\hline \multirow{2}{*}{ Mimican } & $\mathrm{p}$-value & 0.96 & 0.24 & 0.07 & 0.63 \\
\hline & GRA-CV & 0.67 & 0.70 & 0.67 & 0.68 \\
\hline \multirow{2}{*}{ IGFBP7 } & $\mathrm{p}$-value & 0.50 & 0.90 & 0.11 & 0.74 \\
\hline & GRA-CV & 0.70 & 0.73 & 0.70 & 0.71 \\
\hline \multirow{2}{*}{ PLGF/SFLT } & $\mathrm{p}$-value & 0.67 & 0.58 & 0.20 & 0.87 \\
\hline & GRA-CV & 0.71 & 0.73 & 0.71 & 0.72 \\
\hline
\end{tabular}

Table 6.2: Results of the interaction (biomarker $\times$ medication) coefficient $\beta$ tests using weighted logisticGEE models (adjusted for age, gender, coronary artery disease as main cause of HF , Charlson Score, left ventricle ejection fraction, kidney disease, rales, systolic blood pressure, medication, and biomarker in logarithmic form) and their corresponding GRA-CVs.

$\mathrm{p}$-value: $\mathrm{p}$-value of the interaction coefficient $\beta$ tests in weighted logistic-GEE models. 
We note that in our analysis for each medication we have 21 interaction tests. Therefore, due to the multiple testing, at a 5 percent level of significance, the Bonferroni correction suggests to reject the null hypothesis $\left(\mathrm{H}_{0}: \beta=0\right)$ for each test with the $\mathrm{p}$-value less than $0.05 / 21=0.0024$. However, since in this study the results are regarded as hypothesis generating, we consider the interaction tests with p-value less than 0.05 as being significant. Moreover, we note that, $\mathrm{p}$-value adjustments may raise several practical objections [34]. For example you may increase the chance of making a type II error [101, 117].
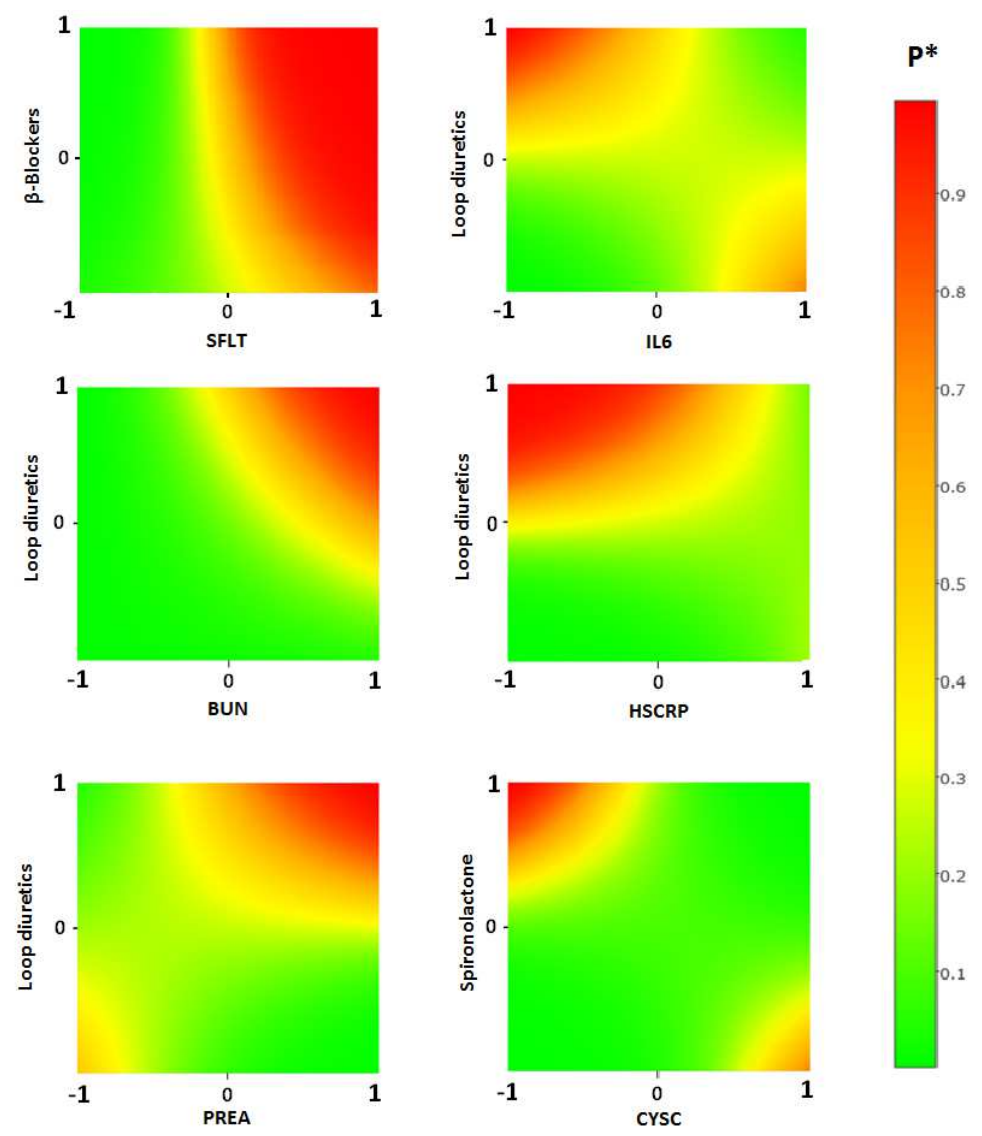

Figure 6.3: Effect of different levels of HF medications and biomarkers on risk of HF hospitalization or death.

${ }^{*} \mathrm{P}$ : Probability of HF hospitalization or death in a month. Range of biomarkers (in logarithmic form) and medications are standardized between -1 and 1 , for the range of the biomarker and medications concentration in our population. 
Chapter 6. Novel Concept to Guide Systolic Heart Failure Medication by Repeated Biomarker Testing

The results indicate that interaction of i) SFLT and TP1NP with treatment effect of $\beta$-blockers, ii) IL6, BUN, HSCRP and PREA with loop diuretics and iii) CYSC and PLGF with spironolactone were significant. That means that these biomarkers might indicate which medication class is most important to up-titrate or possibly down-titrate for improvement of outcome. No significant interactions between biomarkers and RAS blockers were found.

Figure 6.3 depicts the models with significant biomarker - medication interaction, for which GRA-CV $>0.7$. It suggests a beneficial effect of higher doses of loop diuretics in patients with high IL6 and/or high HSCRP. Higher loop diuretic doses seem to have adverse effects in patients with low HSCRP and/or high BUN and PREA. In contrast, high doses of loop diuretics do not seem to harm in patients with low BUN and PREA values. Spironolactone was associated with better outcome (low risk of HF or death) for patients with high CYSC. It also demonstrates that up-titration of $\beta$-blockers for patients with high SFLT might not decrease the risk of HF or death, but that such patients might be better off with low $\beta$-blockers doses.

\subsection{Discussion}

This study investigated the hypothesis whether biomarkers may be able to predict the response to therapy in HF. Several significant biomarker treatment-effect interactions were found. We consider these results as promising signals which may predict a specific response to therapy in individual patients. It must be noted that this study is purely hypothesis generating regarding a novel approach to personalize medicine in HF with the use of biomarkers. Clearly, the results of this study need to be tested in prospective intervention studies before individualized therapy may be applied to HF patients. But our data provide the first attempt to not just predict outcome, but rather to select specific HF therapy based on individual biomarker patterns with the aim to improve outcome.

The need for such an individualized therapy approach in HF has been raised on many occasions, supported by several facts. But in real-life practice, there is still a very high mortality and morbidity despite drug development in the past decades [138]. Moreover, it is often difficult or sometimes even impossible to establish guideline recommended drug therapy in HF [78, 103]. In such cases, it would be crucial to know which drug may be most important to be given in high doses to improve prognosis and which may be less important. At present, it is impossible to make this decision for an individual patient and HF drugs have been investigated on top of previous established therapies only, i.e. $\beta$-blockers on top of ACE-inhibitors, MRAs on top of both previous drugs. Despite a beneficial effect of these drugs as shown in large trials, not every drug will always have the same benefit in a specific patient or patient subgroup. Given the large heterogeneity of HF patients in terms of aetiology and comorbidities, a 'one-size-fits-all' approach is 
likely not optimal [138]. The dose of loop diuretics is even more so a clinical challenge and is largely intuitive. Guidelines recommend diuretics for symptom relief of congestion and recommend to lower diuretics whenever possible [103], but in many instances, they are inappropriately withheld or maintained because of fear of renal dysfunction or fear of decompensation, respectively.

\subsubsection{Considerations regarding statistics}

This investigation is highly strengthened by the fact that both the covariates (i.e. biomarkers at the beginning of a given time interval, medication and clinical covariates) as well as the outcome (i.e. HF hospitalization or death at the end of a given time interval), have been measured at multiple points in time. These multiple time-points help to make the interactions we were investigating clinically relevant, by only looking into the treatment effect that occurred after the measurement of the biomarker in more than just one single time point. However, the difficulty with investigating repeated measurements lies within the fact that the outcomes for a single patient are correlated, because a patient with a hospitalization is prone to suffer from a re-hospitalization or die. Moreover, repeated measurements of biomarkers, medication and other covariates are correlated as well. Therefore, the correlation between outcomes and variation of covariates in time needs to be taken into account for proper analysis.

One approach available for analyzing such data is using survival analysis methods, like time-dependent Cox regression models or recurrent analysis methods such as the Prentice, Williams and Peterson model [106]. However, these methods take into account the values of time-dependent covariates only at the time of events (e.g., time of death or HF hospitalization) and the covariate values between events are disregarded, which was not acceptable to address the objective of this analysis. More proper alternative methods are longitudinal analysis methods [76] that not only take into account correlation in hospitalizations but also can involve more information about the variation of biomarkers and medications and their interactions over time.

Among longitudinal methods, the GEE models [47, 147, 148] have become very popular regression methods in medical studies $[2,68,93,123,131]$. The most attractive property of GEE models is that the resulting estimation of regression coefficients of those models is easy to interpret, especially for binary outcomes. Moreover, applying GEE models, scholars may hypothesize different structures of correlation between outcomes, but the resulting estimation of regression coefficients of GEE models is consistent and asymptotically normal, even when the correlation structure is imprecisely specified $[147,148]$. Another advantage of GEE models for binary outcomes (logistic-GEE models) is that with the use of GRA not only we can assess the adequacy of the model but also we can assess how the obtained results will generalize to a future data set. 
92

Chapter 6. Novel Concept to Guide Systolic Heart Failure Medication by Repeated Biomarker Testing

Therefore in this study, we applied the weighted logistic-GEE model with its underlying a logistic regression model not only for the first, but also for repeated hospitalizations (or death) to test the interaction of biomarkers with the treatment effects of medications over time.

\subsubsection{Underlying pathways of biomarker drug interactions}

First, we found a significant interaction between SFLT and $\beta$-blockers, suggesting that patients with a high SFLT concentration may have a worse outcome with higher doses of $\beta$-blockers as compared to those on lower doses. Patients with low SFLT levels have a more favorable outcome overall, largely irrespective of $\beta$-blocker dose in our population. This raises the hypothesis that uptitration of $\beta$-blockers should be avoided in patients with high SFLT levels. SFLT is the soluble form of the endothelial -and macrophage-bound VEGF-receptor FLT-1. SFLT is formed after alternative splicing of FLT-1 RNA [124] and acts as a decoy receptor, thereby inhibiting VEGF and PLGF. This is assumed to result in anti-angiogenetic and anti-inflammatory effects [124]. SFLT concentrations are increased in HF [44, 46], myocardial infarction [95], pre-eclampsia and coronary disease [124] and higher concentrations of SFLT are associated with adverse outcome in these disease entities [44, 46, 67, 95, 139]. Higher SFLT is also associated with more severe HF according to NYHA-class and NT-proBNP [44, 67]. Nevertheless, the exact role of SFLT in the pathophysiology of HF and cardiovascular disease is not yet fully unraveled. SFLT-knockout mice developed more overt HF after aortic ligation, but on the other hand administration of adenovirus expressing SFLT-1 caused diastolic dysfunction and decreased vascular density in wildtype mice [124]. Thus, both extremes of SFLT may have negative effects in the pathogenesis of HF, requiring a precise balance in the SFLT/PLGF pathway for adequate homeostasis. Another explanation could be that analogous to natriuretic peptides SFLT production is on itself a protective response to cardiac or vascular injury, but SFLT is associated with worse outcome because it also reflects the presence and magnitude of the injury itself. With regard to the interaction of SFLT with $\beta$-blockers on outcome in our study, it is interesting to note that baseline $\beta$-blockers use was independently associated with a lower baseline SFLT concentration previously [67]. Although this was a cross-sectional finding, it might suggest that $\beta$-blockers lower SFLT, but if this cannot be achieved, high doses of $\beta$-blockers might be less favorable. Another explanation could be that an elevated SFLT reflects an advanced stage of HF where $\beta$-blockers are difficult to uptitrate and might result in (temporary) deterioration. This would mean that other drugs might be given first to improve HF and reduce SFLT and $\beta$-blockers uptitration might be postponed. In addition, $\beta$-blockers have been found to have anti-angiogenetic effects in cancer [99]. Although to the best of our knowledge, such anti-angiogenetic effects of $\beta$ blockers have not been properly investigated in HF, it might be speculated that high levels 
of SFLT acting anti-angiogenetically may cause the potential anti-angiogenetic effects of $\beta$-blockers becoming evident. Obviously, this explanation is speculative and it needs to be investigated in animal studies if there is indeed such an interaction in HF.

Second, there was a significant interaction between CYSC and spironolactone. Thus, patients with a low CYSC had a less favorable prognosis on higher doses of spironolactone compared to those on lower doses, whereas patients with a high CYSC had a better outcome with high versus low doses of spironolactone. We note that the results showed also the same pattern for BUN and creatinine, although the interactions were not significant (Data is not shown). CYSC is associated with inflammation and is the most sensitive marker of renal function in terms of glomerular filtration rate [53]. CYSC is strongly associated with risk of cardiovascular disease (CVD) and adverse outcome in HF, but also in the general population. Despite some biological plausible links between CYSC and CVD and HF, a recent mendelian randomization study found no causative role for CYSC in the development of CVD nor in the development of HF [136]. Nevertheless, it remains a very reliable biomarker of high risk of events and disease progression in HF. We are not aware of any previous publication about the interaction between CYSC and MRAs such as spironolactone. However, we and others previously found a similar treatment interaction with serum creatinine levels $[79,112]$. A possible explanation could be that an impaired renal function reflected by elevated CYSC or creatinine in the light of chronic HF is usually a form of cardio-renal syndrome which can be improved when HF is improved. Our results suggest that in this case, the preferred HF-drug could be spironolactone. MRAs are considered to have anti-fibrotic and anti-inflammatory effects, which can also support the link between CYSC and spironolactone that we found.

Finally, we found 4 biomarkers that interacted significantly with loop diuretic dose and outcome. First, in patients with high levels of BUN, higher doses of loop diuretics (HDLD) were associated with worse outcome, whilst this negative association with HDLD was not observed in patients with low BUN. Similar results were previously found by Testatni et al.[130] evaluating 2,456 patients in the BEST-trial. In that study, HDLD was associated with worse outcome when BUN was $\geq 21 \mathrm{mg} / \mathrm{dl}$, but this was not the case when BUN was low. In fact, after controlling for possible confounders, HDLD actually was associated with improved survival in those with low BUN, but with reduced survival in those with high BUN [129]. This interaction between BUN and loop diuretics was confirmed by Nunez et al [91], who further elaborated on this by adding CA125 to the model, leading to a further specification of subgroups with differential risk associated with HDLD. Also supportive of our findings, higher levels of BUN were previously associated with poor diuretic response in HF patients with acute decompensation [129]. PREA revealed an interaction with loop diuretics in a similar direction - HDLD was associated with worse outcome when PREA was high, whereas HDLD was associated with good outcome when PREA was low. A recent study linked signs of intestinal congestion 
94

Chapter 6. Novel Concept to Guide Systolic Heart Failure Medication by Repeated Biomarker

with elevated right atrial pressures and with cachexia [135]. Additionally, prealbumine was lower in patients with hypoalbuminemia [16] and both low prealbumine itself [77] as well as the presence of hypoalbuminemia [16] were associated with adverse outcome. This supports the idea that patients with low concentrations of PREA could have a benefit of HDLD because low PREA indirectly reflects a state of chronic venous congestion. Additionally, both low PREA [77] and cachexia in HF [135] are linked with increased inflammation, linking this interaction also to the inflammation markers. In this regard, two markers of inflammation - HSCRP and IL6 - showed similar patterns of interaction with loop diuretics on outcome in the present analysis. Thus, when inflammation markers were low, HDLD was associated with an increased event risk, whereas when inflammation markers were high, HDLD was associated with a lower risk. Pro-inflammatory activation is linked to congestion [27, 135], but is also considered a major underlying mechanism of $\mathrm{HF}$ progression and a poor prognostic factor, supporting the interaction we found for inflammatory markers and loop diuretics.

\subsubsection{Limitations}

This is a post-hoc exploratory analysis of data from a randomized trial. Therefore, our results must be seen as means to identify potential relationships and to generate hypotheses. Further research - preferably prospective - is needed to confirm these interactions and their mechanisms. In addition, animal studies are required to test the hypotheses raised by our findings. Possible limitations of this analysis are selection bias, reverse causality, and residual confounding factors. Nevertheless, because patients in the TIME-CHF trial were all attempted to be uptitrated on HF drugs either based on clinical symptoms and/or based on NT-proBNP levels, this may limit the selection bias for starting or uptitrating drugs compared to other cohorts. RAS blockers were given in rather high doses in almost all patients and this may also explain why we did not find any interactions between RAS blockers and biomarkers.

\subsubsection{Conclusion}

Our analysis suggests that repeated measurements of biomarkers may be helpful to individually tailor HF treatment, which may optimize the balance between beneficial and adverse effects of HF drugs. Our data provide ground for prospective testing which will be needed before this novel and innovative concept can be adopted. 
Conclusions 
This chapter concludes the thesis. First we summarize the contributions made in the various chapters, to answer the proposed research questions in Chapter 1 . Then we discuss possible directions for future work.

\subsection{Answer to the Research Questions}

This thesis started with the aim of investigating the hypotheses that biomarkers can be useful tools for: (1) predicting the treatment effects of medication in HF patients; (2) guiding the therapy in patients with chronic HF. Therefore the research questions of this thesis were based on these two clinical hypotheses and new statistical methods that can be developed to answer these and other relevant clinical questions.

In this section we return to the research questions that were proposed in Chapter 1 and we discuss each question based on the investigations and obtained results in Chapters 3 to 6.

Research Question 1: Does the NT-proBNP guided therapy reduce repeated HF hospitalizations?

In Chapter 3 we assessed the effect of NT-proBNP-guided therapy compared to symptom-guided therapy on repeated hospitalization events (hospitalizations plus death), within 5.5 years follow-up in the TIME-CHF study. For this, we used PWP-GT modeling as explained in Section 2.2. Regarding to the all-cause hospitalization events, results showed that there was a statistically significant beneficial effect of NT-proBNP-guided therapy on second and third all-cause hospitalization events, predominantly in the younger age group (aged $<75$ years). However, the effect of NT-proBNP-guided therapy as compared to standard therapy on first all-cause hospitalization events was not statistically significant. Regarding to the HF hospitalization event, NT-proBNP-guided therapy showed a beneficial effect on first HF hospitalization event, again predominantly in the younger age group. Only the younger age group has a beneficial effect of NT-proBNP-guided therapy on second HF hospitalization events.

Research Question 2: What is the best cutpoint for dichotomization of continuous variables?

From a statistical and clinical point of view, using dichotomized covariates are often of interest for various reasons. However finding the best cutpoint for dichotomization of continuous variables is still a challenging area of research. In Chapter $\mathbf{4}$ we proposed a 
Bayesian approach to estimate cutpoints for continuous covariates assuming that the dichotomized covariates are related to an outcome variable through a linear regression. The proposed Bayesian approach has the advantage that it can be applied in a multivariate setting. In the multivariate setting with the use of the Bayesian method we may dichotomize all or just a subset of the covariates. To the best of our knowledge this is the first method dealing with multivariate dichotomization with respect to a continuous outcome variable. Unlike most of the available methods, for the proposed Bayesian approach the estimated cutpoint is not limited to the observed values of the covariate and can take any value in the range of the covariate.

We observed in the simulation study that in the both univariate and bivariate settings the performance of the Bayesian approach is satisfactory in terms of estimated mean squared errors and average biases of the estimations. In addition, in the univariate setting the Bayesian approach has a better performance when compared to O'Brien's method.

We applied the proposed Bayesian approach to estimate the cutpoints for BMI, SST2 and Mimecan in the TIME-CHF study with respect to modeling NT-proBNP. We observed that the estimated cutpoints and associations of the dichotomized BMI and SST2 with NT-proBNP were in the same line with the recommended cutpoints and previously observed associations in other studies. We are not aware of any previous study suggesting a cutpoint for dichotomizing Mimecan.

Research Question 3: How to measure the goodness-of-fit and predictive performance of a logistic-GEE model?

Logistic-GEE is one of the most popular models for analyzing clustered binary data. We in fact also applied this model ourselves to address Research Question 4 in this thesis. However, assessing the goodness-of-fit and the predictive performance of logistic-GEE models is problematic due to the fact that the outcomes are correlated within a cluster and no likelihood is available. In addition, the logistic-GEE model disregards the unknown correlation between the outcomes within a cluster and simply models the mean of outcomes.

Several criteria and tests have been proposed for assessing the goodness-of-fit of logistic-GEE models in the literature. However, each of them has at least one of the following shortcomings:

(a) restriction on the number and types of covariates in the model being evaluated,

(b) bias in case of two-class imbalanced data,

(c) difficulty of interpretation, 
(d) a relative range of the criterion values (i.e., the range depends on the number of subjects and number of covariates in the model),

(e) inability to indicate the predictive performance of the model being evaluated.

In Chapter 5 we proposed the Generalized Ranking Accuracy (GRA) as a new measure for assessing the goodness-of-fit as well as the predictive performance of logisticGEE models. We defined GRA as the probability that a randomly selected instance with positive outcome (outcome $=1$ ) is ranked higher than a randomly selected instance with negative outcome (outcome=0) from another cluster. The GRA ranges from 0.0 to 1.0, such that the value 1.0 indicates perfect ranking on the given data (i.e., all instances with positive outcome are ranked higher than all instances with negative outcome from another cluster); and the value 0.0 indicates the opposite scenario. The value 0.5 corresponds to a noninformative model.

The proposed GRA does not suffer from problems (a) to (d). In addition, by using cross-validation, GRA-CV is proposed to use as a predictive performance criterion for logistic-GEE models.

In addition, we compared the behavior of GRA with QICu (as an extension of QIC [97]) as a function of logistic-GEE model complexity. The results showed that unlike GRA, QICu does not perfectly compute model improvements when adding covariates one by one into the logistic-GEE model. As a model-selection criterion, both QICu and GRA-CV (with cluster-out cross-validation) performed well in selection of the covariates in the right order, when the covariates are considerably different in their magnitude of effects, even in the case of two-class imbalanced data. In models for which the covariates are only slightly different in their magnitude of effects, GRA-CV performed better than $\mathrm{QICu}$ when the within-cluster correlation was high (equal to 0.6 or 0.9 ). In general GRA$\mathrm{CV}$ had comparable results, or even better results than QICu, although GRA-CV uses cross-validation, which reduces possibilities for overfitting.

We note that GRA is also applicable to any model for bipartite ranking traind on clustered data.

Research Question 4: Can biomarkers predict treatment effects of medication in patients with systolic HF?

In Chapter 6, we took the first step towards the development of a biomarker-guided treatment algorithm for individually tailoring medical HFrEF therapy. We investigated the interaction between multiple repeatedly measured biomarkers and response to the four most important classes of HF medication regarding the risk of HF hospitalization or death. This has been done with the use of weighted logistic-GEE models. 
The results presented in Chapter 6 confirm the hypothesis that biomarkers may be able to predict response to therapy in HF. Several significant biomarker treatment-effect interactions were found that are promising for potentially predicting response to therapy in individual patients. First, we found a significant interaction between SFLT and $\beta$-blockers suggesting that patients with a high SFLT concentration may have a worse outcome with higher doses of $\beta$-blockers as compared to those on lower doses. Patients with low SFLT levels have a more favorable outcome overall, largely irrespective of $\beta$-blocker dose in our population. This raises the hypothesis that uptitration of $\beta$-blockers should possibly be avoided in patients with high SFLT levels. Second, there was a significant interaction between CYSC and spironolactone. Thus, patients with a low CYSC have a less favorable prognosis on higher doses of spironolactone compared to those on lower doses, whereas patients with a high CYSC have a better outcome with high versus low doses of spironolactone. Finally, we found 4 biomarkers that interacted significantly with the loop diuretic dose with respect to outcome. The results suggested a beneficial effect of higher doses of loop diuretics in patients with high IL6 and/or high HSCRP. Higher loop diuretic doses might have adverse effects in patients with low HSCRP and/or high BUN and PREA. In contrast, high doses of loop diuretics do not seem to harm in patients with low BUN and PREA values.

\subsection{Directions for Future Research}

In this thesis we investigated four research questions in Chapter 3 to 6 in order achieve the objective of the study. Now we discuss some challenging research directions related to each of these chapters.

Chapter 3- In this study, unfortunately, we were not able to assess the effect of NTproBNP-guided therapy on the fourth and consecutive hospitalization events due to lack of a sufficient number of such events. Therefore, repeating this analysis on a larger trial with more number of patients and hospitalization events may help to validate the obtained results. In this thesis we applied PWP-GT model to deal with repeated events; however, there are two shortcomings with this model that needs to be addressed in the future. The PWP-GT model is not able to distinguish death from hospitalization as event. Moreover, there is no method available for calculating the PWP-GT model's power for the whole model as well as for consecutive events. Therefore, as future work, statistical methods need to be developed to assess power of the PWP-GT model and to distinguish death from hospitalization as event. We believe that the PWP-GT model is superior to other methods in dealing with repeated events. This is due to the fact that it takes into account the time between repeated events; it distinguishes the order of events; it takes into consideration the dependence of events within a subject. However, more simulation studies 
or clinical validations are required comparing the PWP-GT model with different methods addressing repeated events.

Chapter 4- We applied a simulation study to evaluate the proposed Bayesian approach for dichotomization in univariate and bivariate settings. However, the simulation study was only applied for limited choices of the parameters. Therefore, it might be interesting to investigate the performance of the proposed Bayesian approach for different choices of the parameters and for models with more than two covariates. The proposed Bayesian approach in this thesis is based on the assumption that the dichotomized covariates are related to a continuous outcome variable through a linear regression model. In future work one may consider dichotomization with respect to a binary outcome through a generalized linear or through non-linear regression models. We point out that it also might be interesting to see the performance of other noninformative priors such as Bernardo's prior [14] and Jeffreys [56] prior. We believe, more work is needed along these directions. Moreover, the proposed Bayesian approach needs to be tested more in the clinical setting to show superiority as compared to previous approaches, and to demonstrate clinical applicability.

Chapter 5- The proposed criterion, GRA, is applicable to any model for bipartite ranking trained on clustered data. However, in this thesis we only evaluated the GRA when using logistic-GEE models. Therefore assessing the GRA as a criterion for goodness-of-fit and predictive performance of other existing bipartite ranking models trained on clustered data might be interesting. In this thesis we proposed GRA-CV as a model selection criterion when we applied a forward model selection technique. This may be assessed for other model selection techniques, e.g., backward or stepwise techniques. In this thesis, in our simulation study we only specified limited values for the parameters and other factors to assess the performances of GRA and GRA-CV. It therefore might be interesting to extend the simulation study considering other values for the size of the data, cluster sizes, magnitude and type of within-cluster correlation, regression coefficients and using different types of cross-validation to evaluate GRA and GRA-CV more precisely. It might also be interesting to extend GRA to models dealing with ordinal or categorical clustered outcomes.

Chapter 6- Although in this thesis several significant biomarker treatment-effect interactions were found, the results of this study still need to be explicitly tested in prospective intervention studies before individualized therapy may be applied to HF patients. In 
addition, animal studies can be required to test the hypotheses raised by our findings.

From the machine learning point of view we may look at the problem in Chapter 6 as a classification problem dealing with correlated outcomes. Therefore, it might be interesting as future work to develop a method in the machine learning domain that can deal with correlated outcomes to get the advantages of available algorithms and techniques in machine learning. We believe, more work is needed along these directions. 


\section{References}

[1] DCRI announces halt to GUIDE-IT trial. https://dcri.org/dcri-announces-haltguide-trial. 2017.

[2] William T Abraham, Gregg C Fonarow, Nancy M Albert, Wendy Gattis Stough, Mihai Gheorghiade, Barry H Greenberg, Christopher M O'Connor, Jie Lena Sun, Clyde W Yancy, and James B Young. Predictors of in-hospital mortality in patients hospitalized for heart failure: insights from the organized program to initiate lifesaving treatment in hospitalized patients with heart failure (OPTIMIZE-HF). Journal of the American College of Cardiology, 52(5):347-356, 2008.

[3] Shivani Agarwal, Thore Graepel, Ralf Herbrich, Sariel Har-Peled, and Dan Roth. Generalization bounds for the area under the ROC curve. Journal of Machine Learning Research, 6:393-425, 2005.

[4] T Ahmad and CM O'connor. Therapeutic implications of biomarkers in chronic heart failure. Clinical Pharmacology \& Therapeutics, 94(4):468-479, 2013.

[5] Tariq Ahmad, Mona Fiuzat, G Michael Felker, and Christopher O'connor. Novel biomarkers in chronic heart failure. Nature Reviews Cardiology, 9(6):347-359, 2012.

[6] Habibul Ahsan, Yu Chen, Faruque Parvez, Lydia Zablotska, Maria Argos, Iftikhar Hussain, Hassina Momotaj, Diane Levy, Zhongqi Cheng, and Vesna Slavkovich. Arsenic exposure from drinking water and risk of premalignant skin lesions in bangladesh: baseline results from the health effects of arsenic longitudinal study. American Journal of Epidemiology, 163(12):1138-1148, 2006.

[7] Hirotugu Akaike. A new look at the statistical model identification. IEEE Transactions on Automatic Control, 19(6):716-723, 1974.

[8] Larry A Allen, Adrian F Hernandez, Christopher M O'Connor, and G Michael Felker. End points for clinical trials in acute heart failure syndromes. Journal of the American College of Cardiology, 53(24):2248-2258, 2009.

[9] Douglas G Altman, Berthold Lausen, Willi Sauerbrei, and Martin Schumacher. Dangers of using optimal cutpoints in the evaluation of prognostic factors. Journal of the National Cancer Institute, 86(11):829-835, 1994. 
[10] Leila DAF Amorim and Jianwen Cai. Modelling recurrent events: a tutorial for analysis in epidemiology. International Journal of Epidemiology, 44(1):324-333, 2015.

[11] Per Kragh Andersen and Richard David Gill. Cox's regression model for counting processes: a large sample study. The Annals of Statistics, 10(4):1100-1120, 1982.

[12] Peter C Austin and Lawrence J Brunner. Inflation of the type i error rate when a continuous confounding variable is categorized in logistic regression analyses. Statistics in Medicine, 23(7):1159-1178, 2004.

[13] Huiman X Barnhart and John M Williamson. Goodness-of-fit tests for GEE modeling with binary responses. Biometrics, 54(2):720-729, 1998.

[14] Jose M Bernardo. Reference posterior distributions for bayesian inference. Journal of the Royal Statistical Society. Series B (Methodological), 41(2):113-147, 1979.

[15] Avrim Blum, Adam Kalai, and John Langford. Beating the hold-out: Bounds for $\mathrm{k}$-fold and progressive cross-validation. In Proceedings of the twelfth annual conference on Computational learning theory, pages 203-208. ACM, 1999.

[16] Juan Luis Bonilla-Palomas, Antonio Luis Gámez-López, Mirian Moreno-Conde, María Cristina López-Ibáñez, Manuel Anguita-Sánchez, Ángel Gallego de la Sacristana, Francisco García-Catalán, and Antonia Villar-Ráez. Hypoalbuminemia in acute heart failure patients: causes and its impact on hospital and long-term mortality. Journal of Cardiac Failure, 20(5):350-358, 2014.

[17] Jeffrey S Borer, Michael Böhm, Ian Ford, Michel Komajda, Luigi Tavazzi, Jose Lopez Sendon, Marco Alings, Esteban Lopez-de Sa, Karl Swedberg, and Shift Investigators. Effect of ivabradine on recurrent hospitalization for worsening heart failure in patients with chronic systolic heart failure: the SHIFT study. European Heart Journal, 33(22):2813.

[18] Leo Breiman, Jerome Friedman, Charles J Stone, and Richard A Olshen. Classification and regression trees. CRC press, 1984.

[19] Hans-Peter Brunner-La Rocca, Peter Theo Buser, Ruth Schindler, Alain Bernheim, Peter Rickenbacher, Matthias Pfisterer, and TIME-CHF-Investigators. Management of elderly patients with congestive heart failuredesign of the trial of intensified versus standard medical therapy in elderly patients with congestive heart failure (TIME-CHF). American Heart Journal, 151(5):949-955, 2006.

[20] George Casella and Roger L Berger. Statistical inference, volume 2. Duxbury Pacific Grove, CA, 2002. 
[21] Jennifer SK Chan. Analysis of correlation structures using generalized estimating equation approach for longitudinal binary data. Journal of Data Science, 12(2):293-305, 2014.

[22] Weiwei Cheng and Eyke Hüllermeier. Combining instance-based learning and logistic regression for multilabel classification. Machine Learning, 76(2):211-225, 2009.

[23] Siddhartha Chib and Edward Greenberg. Understanding the metropolis-hastings algorithm. The American Statistician, 49(4):327-335, 1995.

[24] Mariantonietta Cicoira, Aldo Pietro Maggioni, Roberto Latini, Simona Barlera, Elisa Carretta, Andras Janosi, Jordi Soler Soler, Inder Anand, and Jay N Cohn. Body mass index, prognosis and mode of death in chronic heart failure: results from the valsartan heart failure trial. European Journal of Heart Failure, 9(4):397402, 2007.

[25] Aldo Clerico, Alberto Giannoni, Simona Vittorini, and Michele Emdin. The paradox of low BNP levels in obesity. Heart Failure Reviews, 17(1):81-96, 2012.

[26] Jacob Cohen. The cost of dichotomization. Applied Psychological Measurement, 7(3):249-253, 1983.

[27] Paolo C Colombo, Amanda C Doran, Duygu Onat, Ka Yuk Wong, Myra Ahmad, Hani N Sabbah, and Ryan T Demmer. Venous congestion, endothelial and neurohormonal activation in acute decompensated heart failure: cause or effect? Current Heart Failure Reports, 12(3):215-222, 2015.

[28] Richard J Cook and Jerald Lawless. The statistical analysis of recurrent events. Springer Science \& Business Media, 2007.

[29] Shannon M Dunlay, Margaret M Redfield, Susan A Weston, Terry M Therneau, Kirsten Hall Long, Nilay D Shah, and Véronique L Roger. Hospitalizations after heart failure diagnosis: a community perspective. Journal of the American College of Cardiology, 54(18):1695-1702, 2009.

[30] Lawrence J Emrich and Marion R Piedmonte. A method for generating highdimensional multivariate binary variates. The American Statistician, 45(4):302304, 1991.

[31] Scott Evans and Lingling Li. A comparison of goodness of fit tests for the logistic GEE model. Statistics in Medicine, 24(8):1245-1261, 2005. 
[32] Scott R Evans and David W Hosmer. Goodness of fit tests for logistic GEE models: Simulation results. Communications in Statistics-Simulation and Computation, 33(1):247-258, 2004.

[33] David Faraggi and Richard Simon. A simulation study of cross-validation for selecting an optimal cutpoint in univariate survival analysis. Statistics in Medicine, 15(20):2203-2213, 1996.

[34] Ronald J Feise. Do multiple outcome measures require p-value adjustment? BMC Medical Research Methodology, 2(1):8, 2002.

[35] G Michael Felker, Tariq Ahmad, Kevin J Anstrom, Kirkwood F Adams, Lawton S Cooper, Justin A Ezekowitz, Mona Fiuzat, Nancy Houston-Miller, James L Januzzi, Eric S Leifer, et al. Rationale and design of the GUIDE-IT study: guiding evidence based therapy using biomarker intensified treatment in heart failure. JACC: Heart Failure, 2(5):457-465, 2014.

[36] G Michael Felker, Vic Hasselblad, Adrian F Hernandez, and Christopher M O'Connor. Biomarker-guided therapy in chronic heart failure: a meta-analysis of randomized controlled trials. American Heart Journal, 158(3):422-430, 2009.

[37] António Ferreira, Paulo Bettencourt, Dias Paula, Manuel Pestana, Paula Serrão, Patrício Soares-da silva, and Mário Cerqueira-Gomes. Neurohormonal activation, the renal dopaminergic system and sodium handling in patients with severe heart failure under vasodilator therapy. Clinical Science, 100(5):557-566, 2001.

[38] Alan E Gelfand and Adrian FM Smith. Sampling-based approaches to calculating marginal densities. Journal of the American Statistical Association, 85(410):398409, 1990.

[39] John Geweke et al. Evaluating the accuracy of sampling-based approaches to the calculation of posterior moments, volume 196. Federal Reserve Bank of Minneapolis, Research Department Minneapolis, MN, USA, 1991.

[40] Walter R Gilks. Markov chain Monte Carlo. Encyclopedia of Biostatistics, 2005.

[41] Robert J Glynn and Julie E Buring. Ways of measuring rates of recurrent events. BMJ: British Medical Journal, 312(7027):364, 1996.

[42] Michael Goldstein and David Wooff. Bayes linear statistics, theory and methods, volume 716. John Wiley \& Sons, 2007.

[43] Major Greenwood and G Udny Yule. An inquiry into the nature of frequency distributions representative of multiple happenings with particular reference to the 
occurrence of multiple attacks of disease or of repeated accidents. Journal of the Royal Statistical Society, 83(2):255-279, 1920.

[44] Damien Gruson, Michel P Hermans, Benjamin Ferracin, Sylvie A Ahn, and Michel F Rousseau. SFLT-1 in heart failure: relation with disease severity and biomarkers. Scandinavian Journal of Clinical and Laboratory Investigation, 76(5):411-416, 2016.

[45] Zhenchao Guo, Thomas M Gill, and Heather G Allore. Modeling repeated time-toevent health conditions with discontinuous risk intervals: an example of a longitudinal study of functional disability among older persons. Methods of Information in Medicine, 47(2):107, 2008.

[46] Muhammad Hammadah, Vasiliki V Georgiopoulou, Andreas P Kalogeropoulos, Malory Weber, Xi Wang, Michael A Samara, Yuping Wu, Javed Butler, and WH Wilson Tang. Elevated soluble fms-like tyrosine kinase-1 and placental-like growth factor levels are associated with development and mortality risk in heart failure. Circulation: Heart Failure, 9(1):e002115, 2016.

[47] James A Hanley, Abdissa Negassa, and Janet E Forrester. Statistical analysis of correlated data using generalized estimating equations: an orientation. American Journal of Epidemiology, 157(4):364-375, 2003.

[48] James W Hardin. Generalized estimating equations (GEE). Wiley Online Library, 2005.

[49] Joseph M Hilbe, JW Hardin, and HW Hardin. Generalized estimating equations. CRC Press, 2003.

[50] Kalon KL Ho, Joan L Pinsky, William B Kannel, and Daniel Levy. The epidemiology of heart failure: the Framingham study. Journal of the American College of Cardiology, 22(4):A6-A13, 1993.

[51] Richard Hobbs. Clinical burden and health service challenges of chronic heart failure. Br J Gen Pract, 60(577):611-615, 2010.

[52] Robert V Hogg and Allen T Craig. Introduction to mathematical statistics. $\left(5^{\text {th }}\right.$ edition). Upper Saddle River, New Jersey: Prentice Hall, 1995.

[53] R Hojs, S Bevc, R Ekart, M Gorenjak, and L Puklavec. Serum cystatin C-based equation compared to serum creatinine-based equations for estimation of glomerular filtration rate in patients with chronic kidney disease. Clinical Nephrology, 70(1):10-17, 2008. 
[54] Yijian Huang and Ying Qing Chen. Marginal regression of gaps between recurrent events. Lifetime Data Analysis, 9(3):293-303, 2003.

[55] M Katherine Hutchinson and Matthew C Holtman. Analysis of count data using poisson regression. Research in Nursing \& Health, 28(5):408-418, 2005.

[56] Joseph G Ibrahim and Purushottam W Laud. On Bayesian analysis of generalized linear models using Jeffreys's prior. Journal of the American Statistical Association, 86(416):981-986, 1991.

[57] Mi Jin Jang. Working correlation selection in generalized estimating equations. Ph.D. dissertation. The University of Iowa, 2011.

[58] James L Januzzi, Carlos A Camargo, Saif Anwaruddin, Aaron L Baggish, Annabel A Chen, Daniel G Krauser, Roderick Tung, Renee Cameron, J Tobias Nagurney, Claudia U Chae, et al. The N-terminal Pro-BNP investigation of dyspnea in the emergency department (PRIDE) study. The American Journal of Cardiology, 95(8):948-954, 2005.

[59] James L Januzzi, Alexandre Mebazaa, and Salvatore Di Somma. ST2 and prognosis in acutely decompensated heart failure: the international ST2 consensus panel. The American Journal of Cardiology, 115(7):26B-31B, 2015.

[60] Patrick Jourdain, Guillaume Jondeau, François Funck, Pascal Gueffet, Alain Le Helloco, Erwan Donal, Jean F Aupetit, Marie C Aumont, Michel Galinier, and Jean C Eicher. Plasma brain natriuretic peptide-guided therapy to improve outcome in heart failure: the STARS-BNP multicenter study. Journal of the American College of Cardiology, 49(16):1733-1739, 2007.

[61] Beat A Kaufmann, Kaatje Goetschalckx, Son Y Min, Micha T Maeder, Urs Bucher, Fabian Nietlispach, Alain M Bernheim, Matthias E Pfisterer, and HansPeter Brunner-La Rocca. Improvement in left ventricular ejection fraction and reverse remodeling in elderly heart failure patients on intense NT-proBNP-guided therapy. International Journal of Cardiology, 191:286-293, 2015.

[62] Min-Seok Kim, Tae-Dong Jeong, Seung-Bong Han, Won-Ki Min, and Jae-Joong Kim. Role of soluble st 2 as a prognostic marker in patients with acute heart failure and renal insufficiency. Journal of Korean Medical Science, 30(5):569-575, 2015.

[63] David G Kleinbaum and Mitchel Klein. Survival analysis: a self-learning text. Springer Science \& Business Media, 2006. 
[64] Daniel G Krauser, Donald M Lloyd-Jones, Claudia U Chae, Renee Cameron, Saif Anwaruddin, Aaron L Baggish, Annabel Chen, Roderick Tung, and James L Januzzi. Effect of body mass index on natriuretic peptide levels in patients with acute congestive heart failure: a probnp investigation of dyspnea in the emergency department (pride) substudy. American Heart Journal, 149(4):744-750, 2005.

[65] Miloš Kubánek, Kevin M Goode, Věra Lánská, Andrew L Clark, and John GF Cleland. The prognostic value of repeated measurement of n-terminal pro-b-type natriuretic peptide in patients with chronic heart failure due to left ventricular systolic dysfunction. European Journal of Heart Failure, 11(4):367-377, 2009.

[66] Bonnie Ky, Benjamin French, Kristin McCloskey, J Eduardo Rame, Erin McIntosh, Puja Shahi, Daniel Dries, WH Wilson Tang, Alan HB Wu, James C Fang, Rebecca Boxer, Nancy K Sweitzer, Wayne Levy, Lee Goldberg, Mariell Jessup, and Thomas P Cappola. High-sensitivity ST2 for prediction of adverse outcomes in chronic heart failure. Circulation: Heart Failure, 4(2):180-187, 2011.

[67] Bonnie Ky, Benjamin French, Kosha Ruparel, Nancy K Sweitzer, James C Fang, Wayne C Levy, Douglas B Sawyer, and Thomas P Cappola. The vascular marker soluble FMS-like tyrosine kinase 1 is associated with disease severity and adverse outcomes in chronic heart failure. Journal of the American College of Cardiology, 58(4):386-394, 2011.

[68] Jennifer Elston Lafata, Manel Pladevall, George Divine, Melissa Ayoub, and Edward F Philbin. Are there race/ethnicity differences in outpatient congestive heart failure management, hospital use, and mortality among an insured population? Medical Care, 42(7):680-689, 2004.

[69] John G Lainchbury, Richard W Troughton, Kim M Strangman, Christopher M Frampton, Anna Pilbrow, Timothy G Yandle, Amjad K Hamid, M Gary Nicholls, and A Mark Richards. N-terminal pro B-type natriuretic peptide-guided treatment for chronic heart failure: results from the BATTLESCARRED (NT-proBNPassisted treatment to lessen serial cardiac readmissions and death) trial. Journal of the American College of Cardiology, 55(1):53-60, 2009.

[70] J Richard Landis and Gary G Koch. The measurement of observer agreement for categorical data. Biometrics, 33(1):159-174, 1977.

[71] Erich Leo Lehmann and George Casella. Theory of point estimation. Springer Science \& Business Media, 2006.

[72] Daniel Levy, Satish Kenchaiah, Martin G Larson, Emelia J Benjamin, Michelle J Kupka, Kalon KL Ho, Joanne M Murabito, and Ramachandran S Vasan. Long-term 
trends in the incidence of and survival with heart failure. New England Journal of Medicine, 347(18):1397-1402, 2002.

[73] Kung Yee Liang and Scott L Zeger. Longitudinal data analysis using generalized linear models. Biometrika, 73(1):13-22, 1986.

[74] Danyu Y Lin and Lee-Jen Wei. The robust inference for the cox proportional hazards model. Journal of the American Statistical Association, 84(408):10741078, 1989.

[75] Xinhua Liu. Classification accuracy and cut point selection. Statistics in Medicine, 31(23):2676-2686, 2012.

[76] Joseph J Locascio and Alireza Atri. An overview of longitudinal data analysis methods for neurological research. Dementia and geriatric cognitive disorders extra, 1(1):330-357, 2011.

[77] Patrícia Lourenço, Sérgio Silva, Fernando Friões, Margarida Alvelos, Marta Amorim, Marta Couto, Paulo Torres-Ramalho, João Tiago Guimarães, José Paulo Araújo, and Paulo Bettencourt. Low prealbumin is strongly associated with adverse outcome in heart failure. Heart, 100(22):1780-1785, 2014.

[78] Aldo P Maggioni, Stefan D Anker, Ulf Dahlström, Gerasimos Filippatos, Piotr Ponikowski, Faiez Zannad, Offer Amir, Ovidiu Chioncel, Marisa Crespo Leiro, and Jaroslaw Drozdz. Are hospitalized or ambulatory patients with heart failure treated in accordance with european society of cardiology guidelines? evidence from 12,440 patients of the ESC heart failure long-term registry. European Journal of Heart Failure, 15(10):1173-1184, 2013.

[79] Alan Maisel, Yang Xue, Dirk J Van Veldhuisen, Adriaan A Voors, Tiny Jaarsma, Peter S Pang, Javed Butler, Bertram Pitt, Paul Clopton, and Rudolf A De Boer. Effect of spironolactone on 30-day death and heart failure rehospitalization (from the coach study). The American Journal of Cardiology, 114(5):737-742, 2014.

[80] Alan S Maisel, Padma Krishnaswamy, Richard M Nowak, James McCord, Judd E Hollander, Philippe Duc, Torbjørn Omland, Alan B Storrow, William T Abraham, Alan HB Wu, Paul Clopton, Philippe G Steg, Arne Westheim, Catherine Wold Knudsen, Alberto Perez, Radmila Kazanegra, Howard C Herrmann, and Peter A McCullough. Rapid measurement of B-type natriuretic peptide in the emergency diagnosis of heart failure. New England Journal of Medicine, 347(3):161167, 2002. 
[81] Frederick A Masoudi, Edward P Havranek, and Harlan M Krumholz. The burden of chronic congestive heart failure in older persons: magnitude and implications for policy and research. Heart Failure Reviews, 7(1):9-16, 2002.

[82] Madhu Mazumdar and Jill R Glassman. Categorizing a prognostic variable: review of methods, code for easy implementation and applications to decision-making about cancer treatments. Statistics in Medicine, 19(1):113-132, 2000.

[83] John J McMurray and Simon Stewart. Epidemiology, aetiology, and prognosis of heart failure. Heart, 83(5):596-602, 2000.

[84] John JV McMurray, Stamatis Adamopoulos, Stefan D Anker, Angelo Auricchio, Michael Böhm, Kenneth Dickstein, Volkmar Falk, Gerasimos Filippatos, Cândida Fonseca, Miguel Angel Gomez-Sanchez, et al. ESC guidelines for the diagnosis and treatment of acute and chronic heart failure 2012: The task force for the diagnosis and treatment of acute and chronic heart failure 2012 of the European Society of Cardiology. developed in collaboration with the heart failure association (HFA) of the ESC. European journal of Heart Failure, 14(8):803-869, 2012.

[85] Arend Mosterd and Arno W Hoes. Clinical epidemiology of heart failure. Heart, 93(9):1137-1146, 2007.

[86] Shweta R Motiwala and James L Januzzi Jr. Using biomarkers to guide heart failure management: current perspectives and future directions. Cardiology in Review, 21(3):127-134, 2013.

[87] Stefano Muzzarelli, Micha Tobias Maeder, Stefan Toggweiler, Hans Rickli, Fabian Nietlispach, Barbara Julius, Thilo Burkard, Matthias Emil Pfisterer, Hans-Peter Brunner-La Rocca, and TIME-CHF Investigators. Frequency and predictors of hyperkalemia in patients 60 years of age with heart failure undergoing intense medical therapy. The American Journal of Cardiology, 109(5):693-698, 2012.

[88] Brent N Reed and Carla A Sueta. A practical guide for the treatment of symptomatic heart failure with reduced ejection fraction (HFrEF). Current Cardiology Reviews, 11(1):23-32, 2015.

[89] Vijaiganesh Nagarajan, Luke Kohan, Eric Holland, Ellen C Keeley, and Sula Mazimba. Obesity paradox in heart failure: a heavy matter. ESC Heart Failure, 3(4):227-234, 2016.

[90] Hanyu Ni. Prevalence of self-reported heart failure among us adults: results from the 1999 national health interview survey. American Heart Journal, 146(1):121$128,2003$. 
[91] Julio Núñez, Eduardo Núñez, Gema Miñana, Vicent Bodí, Gregg C Fonarow, Vicente Bertomeu-González, Patricia Palau, Pilar Merlos, Silvia Ventura, Francisco J Chorro, Pau Llácer, and Juan Sanchis. Differential mortality association of loop diuretic dosage according to blood urea nitrogen and carbohydrate antigen 125 following a hospitalization for acute heart failure. European Journal of Heart Failure, 14(9):974-984, 2012.

[92] Sean M O'Brien. Cutpoint selection for categorizing a continuous predictor. Biometrics, 60(2):504-509, 2004.

[93] Daniel P O’Connor, Molly S Bray, Brian K McFarlin, Mary H Sailors, Kenneth J Ellis, and Andrew S Jackson. Generalized equations for estimating DXA percent fat of diverse young women and men: the TIGER study. Medicine and science in sports and exercise, 42(10):1959, 2010.

[94] A O'Hagan and J Forster. Kendall's advanced theory of statistics: Bayesian inference. Arnold. London, UK, 2004.

[95] Kenji Onoue, Shiro Uemura, Yukiji Takeda, Satoshi Somekawa, Hajime Iwama, Taku Nishida, Yoshinobu Morikawa, Hitoshi Nakagawa, Takeshi Tsutsumi, and Ji Hee Sung. Usefulness of soluble FMS-like tyrosine kinase-1 as a biomarker of acute severe heart failure in patients with acute myocardial infarction. The American Journal of Cardiology, 104(11):1478-1483, 2009.

[96] Alberto Palazzuoli, Giovanni Antonelli, Ilaria Quatrini, and Ranuccio Nuti. Natriuretic peptides in heart failure: where we are, where we are going. Internal and Emergency Medicine, 6(1):63-68, 2011.

[97] Wei Pan. Akaike's information criterion in generalized estimating equations. Biometrics, 57(1):120-125, 2001.

[98] Wei Pan. Goodness-of-fit tests for GEE with correlated binary data. Scandinavian Journal of Statistics, 29(1):101-110, 2002.

[99] E Pasquier, J Street, C Pouchy, M Carre, AJ Gifford, J Murray, MD Norris, T Trahair, $\mathrm{N}$ Andre, and $\mathrm{M}$ Kavallaris. $\beta$-blockers increase response to chemotherapy via direct antitumour and anti-angiogenic mechanisms in neuroblastoma. British Journal of Cancer, 108(12):2485-2494, 2013.

[100] Jennie Pearce and Simon Ferrier. Evaluating the predictive performance of habitat models developed using logistic regression. Ecological Modelling, 133(3):225$245,2000$. 
[101] Thomas V Perneger. Whats wrong with bonferroni adjustments. BMJ: British Medical Journal, 316(7139):1236, 1998.

[102] Matthias Pfisterer, Peter Buser, Hans Rickli, Marc Gutmann, Paul Erne, Peter Rickenbacher, André Vuillomenet, Urs Jeker, Paul Dubach, and Hansjürg Beer. BNP-guided vs symptom-guided heart failure therapy: the trial of intensified vs standard medical therapy in elderly patients with congestive heart failure (TIMECHF) randomized trial. JAMA, 301(4):383-392, 2009.

[103] Piotr Ponikowski, Adriaan A Voors, Stefan D Anker, Héctor Bueno, John GF Cleland, Andrew JS Coats, Volkmar Falk, José Ramón González-Juanatey, Veli-Pekka Harjola, Ewa A Jankowska, et al. 2016 esc guidelines for the diagnosis and treatment of acute and chronic heart failure: The task force for the diagnosis and treatment of acute and chronic heart failure of the european society of cardiology (ESC) developed with the special contribution of the heart failure association (HFA) of the esc. European Heart Journal, 37(27):2129-2200, 2016.

[104] Pramote Porapakkham, Pornwalee Porapakkham, Hendrik Zimmet, Baki Billah, and Henry Krum. B-type natriuretic peptide-guided heart failure therapy: a metaanalysis. Archives of Internal Medicine, 170(6):507-514, 2010.

[105] John S Preisser, Kurt K Lohman, and Paul J Rathouz. Performance of weighted estimating equations for longitudinal binary data with drop-outs missing at random. Statistics in Medicine, 21(20):3035-3054, 2002.

[106] Ross L Prentice, Benjamin J Williams, and Arthur V Peterson. On the regression analysis of multivariate failure time data. Biometrika, 68(2):373-379, 1981.

[107] Erik Pulkstenis and Timothy J Robinson. Two goodness-of-fit tests for logistic regression models with continuous covariates. Statistics in Medicine, 21(1):79-93, 2002.

[108] David R Ragland. Dichotomizing continuous outcome variables: dependence of the magnitude of association and statistical power on the cutpoint. Epidemiology, 3(5):434-440, 1992.

[109] Howard Raiffa and Robert Schlaifer. Applied statistical decision theory. Harvard University Graduate School of Business Administration (Division of Research); Bailey \& Swinfen, 1961.

[110] Margaret M Redfield, Steven J Jacobsen, John C Burnett Jr, Douglas W Mahoney, Kent R Bailey, and Richard J Rodeheffer. Burden of systolic and diastolic ventricular dysfunction in the community: appreciating the scope of the heart failure epidemic. JAMA, 289(2):194-202, 2003. 
[111] J Remes, H Miettinen, A Reunanen, and K Pyörälä. Validity of clinical diagnosis of heart failure in primary health care. European Heart Journal, 12(3):315-321, 1991.

[112] Brunner-La Rocca, Christian Knackstedt, Luc Eurlings, Vinzent Rolny, Friedemann Krause, Matthias E Pfisterer, Daniel Tobler, Peter Rickenbacher, Micha T Maeder, et al. Impact of worsening renal function related to medication in heart failure. European Journal of Heart Failure, 17(2):159-168, 2015.

[113] Jennifer K Rogers, John JV McMurray, Stuart J Pocock, Faiez Zannad, Henry Krum, Dirk J van Veldhuisen, Karl Swedberg, Harry Shi, John Vincent, and Bertram Pitt. Eplerenone in patients with systolic heart failure and mild symptoms analysis of repeat hospitalizations. Circulation, 126(19):2317-2323, 2012.

[114] Jennifer K Rogers, Stuart J Pocock, John JV McMurray, Christopher B Granger, Eric L Michelson, Jan Östergren, Marc A Pfeffer, Scott D Solomon, Karl Swedberg, and Salim Yusuf. Analysing recurrent hospitalizations in heart failure: a review of statistical methodology, with application to charm-preserved. European Journal of Heart Failure, 16(1):33-40, 2014.

[115] Emilio Ros. Nuts and novel biomarkers of cardiovascular disease. The American Journal of Clinical Nutrition, 89(5):1649S-1656S, 2009.

[116] Wayne Rosamond, Katherine Flegal, Gary Friday, Karen Furie, Alan Go, Kurt Greenlund, Nancy Haase, Michael Ho, Virginia Howard, Bret Kissela, Steven Kittner, Donald Lloyd-Jones, Mary McDermott, James Meigs, Claudia Moy, Graham Nichol, Christopher J O’Donnell, Veronique Roger, John Rumsfeld, Paul Sorlie, Julia Steinberger, Thomas Thom, Sylvia Wasserthiel-Smoller, and Yuling Hong. Heart disease and stroke statistics-2007 update. Circulation, 115(5):e69e171, 2007.

[117] Kenneth J Rothman. No adjustments are needed for multiple comparisons. Epidemiology, pages 43-46, 1990.

[118] Sandra Sanders-van Wijk, Micha T Maeder, Fabian Nietlispach, Hans Rickli, Werner Estlinbaum, Paul Erne, Peter Rickenbacher, Martin Peter, Matthias P Pfisterer, and Hans-Peter Brunner-La Rocca. Long-term results of intensified, N-Terminal-pro-B-type Natriuretic Peptide-guided versus symptom-guided treatment in elderly patients with heart failure five-year follow-up from TIME-CHF. Circulation: Heart Failure, 7(1):131-139, 2014.

[119] Sandra Sanders-van Wijk, Stefano Muzzarelli, Michael Neuhaus, Stephanie Kiencke, Micha Maeder, Werner Estlinbaum, Daniel Tobler, Kurt Mayer, Paul 
Erne, and Matthias E Pfisterer. Safety and tolerability of intensified, N-Terminal pro Brain Natriuretic Peptide-guided compared with standard medical therapy in elderly patients with congestive heart failure: results from TIME-CHF. European Journal of Heart Failure, 15(8):910-918, 2013.

[120] Sandra Sanders-van Wijk, Vanessa van Empel, Nasser Davarzani, Micha T Maeder, Rolf Handschin, Matthias E Pfisterer, Brunner-La Rocca, et al. Circulating biomarkers of distinct pathophysiological pathways in heart failure with preserved vs. reduced left ventricular ejection fraction. European Journal of Heart Failure, 17(10):1006-1014, 2015.

[121] Gianluigi Savarese, Bruno Trimarco, Santo Dellegrottaglie, Maria Prastaro, Francesco Gambardella, Giuseppe Rengo, Dario Leosco, and Pasquale PerroneFilardi. Natriuretic peptide-guided therapy in chronic heart failure: a meta-analysis of 2,686 patients in 12 randomized trials. PloS one, 8(3):e58287, 2013.

[122] DD Schocken, EJ Benjamin, GC Fonarow, HM Krumholz, D Levy, GA Mensah, J Narula, ES Shor, JB Young, and Y Hong. American heart association council on epidemiology and prevention; american heart association council on clinical cardiology; american heart association council on cardiovascular nursing; american heart association council on high blood pressure research; quality of care and outcomes research interdisciplinary working group. Functional Genomics and Translational Biology Interdisciplinary Working Group. Prevention of heart failure: a scientific statement from the American Heart Association Councils on Epidemiology and Prevention, Clinical Cardiology, Cardiovascular Nursing, and High Blood Pressure Research, pages 2544-2565, 2008.

[123] Samantha R Seals and Inmaculada B Aban. Analysis of the 17-segment left ventricle model using generalized estimating equations. Journal of Nuclear Cardiology, 23(5):1110-1111, 2016.

[124] Ayako Seno, Yukiji Takeda, Masaru Matsui, Aya Okuda, Tomoya Nakano, Yasuki Nakada, Takuya Kumazawa, Hitoshi Nakagawa, Taku Nishida, and Kenji Onoue. Suppressed production of soluble fms-like tyrosine kinase-1 contributes to myocardial remodeling and heart failurenovelty and significance. Hypertension, 68(3):678-687, 2016.

[125] Monica R Shah, Robert M Califf, Anju Nohria, Manju Bhapkar, Margaret Bowers, Donna M Mancini, Mona Fiuzat, Lynne W Stevenson, and Christopher M O'Connor. The starbrite trial: a randomized, pilot study of B-type natriuretic peptide-guided therapy in patients with advanced heart failure. Journal of Cardiac Failure, 17(8):613-621, 2011. 
[126] Samuel Sanford Shapiro and Martin B Wilk. An analysis of variance test for normality (complete samples). Biometrika, 52(3/4):591-611, 1965.

[127] Simon Stewart, Andrew Jenkins, Scot Buchan, Alistair McGuire, Simon Capewell, and John JJV McMurray. The current cost of heart failure to the national health service in the UK. European Journal of Heart Failure, 4(3):361-371, 2002.

[128] R Core Team. R: A language and environment for statistical computing. https://www.r-project.org/. Vienna, Austria: R foundation for statistical computing, 2016.

[129] Jozine M ter Maaten, Mattia AE Valente, Marco Metra, Noemi Bruno, Christopher M O’Connor, Piotr Ponikowski, John R Teerlink, Gad Cotter, Beth Davison, and John G Cleland. A combined clinical and biomarker approach to predict diuretic response in acute heart failure. Clinical Research in Cardiology, 105(2):145$153,2016$.

[130] Jeffrey M Testani, Thomas P Cappola, Colleen M Brensinger, Richard P Shannon, and Stephen E Kimmel. Interaction between loop diuretic-associated mortality and blood urea nitrogen concentration in chronic heart failure. Journal of the American College of Cardiology, 58(4):375-382, 2011.

[131] Marita G Titler, Gwenneth A Jensen, Joanne McCloskey Dochterman, Xian Jin Xie, Mary Kanak, David Reed, and Leah L Shever. Cost of hospital care for older adults with heart failure: medical, pharmaceutical, and nursing costs. Health Services Research, 43(2):635-655, 2008.

[132] Anestis Touloumis. Simulating correlated binary and multinomial responses under marginal model specification: The simcormultres package. The R Journal, 2016.

[133] Richard W Troughton, Christopher M Frampton, Timothy G Yandle, Eric A Espine, M Gary Nicholls, and A Mark Richards. Treatment of heart failure guided by plasma aminoterminal brain natriuretic peptide (n-bnp) concentrations. The Lancet, 355(9210):1126-1130, 2000.

[134] Gisela Tunes-da-Silva and John P Klein. Cutpoint selection for discretizing a continuous covariate for generalized estimating equations. Computational Statistics \& Data Analysis, 55(1):226-235, 2011.

[135] Miroslava Valentova, Stephan von Haehling, Juergen Bauditz, Wolfram Doehner, Nicole Ebner, Tarek Bekfani, Sebastian Elsner, Veronika Sliziuk, Nadja Scherbakov, and Ján Murín. Intestinal congestion and right ventricular dysfunction: a link with appetite loss, inflammation, and cachexia in chronic heart failure. European Heart Journal, 37(21):1684-1691, 2016. 
[136] Sander W Van Der Laan, Tove Fall, Aicha Soumaré, Alexander Teumer, Sanaz Sedaghat, Jens Baumert, Delilah Zabaneh, Jessica van Setten, Ivana Isgum, Tessel E Galesloot, et al. Cystatin C and cardiovascular disease: a mendelian randomization study. Journal of the American College of Cardiology, 68(9):934-945, 2016.

[137] Roland RJ van Kimmenade and James L Januzzi. Emerging biomarkers in heart failure. Clinical Chemistry, 58(1):127-138, 2012.

[138] Adriaan Voors, Stefan D Anker, John G Cleland, Kenneth Dickstein, Gerasimos Filippatos, Pim van der Harst, Hans L Hillege, Chim C Lang, Jozine M ter Maaten, and Leong Ng. A systems BIOlogy study to TAilored Treatment in Chronic Heart Failure: rationale, design, and baseline characteristics of BIOSTAT-CHF. European Journal of Heart Failure, 18(6):716-716, 2016.

[139] Esther Vorovich, Benjamin French, Bonnie Ky, Lee Goldberg, James C Fang, Nancy K Sweitzer, and Thomas P Cappola. Biomarker predictors of cardiac hospitalization in chronic heart failure: a recurrent event analysis. Journal of Cardiac Failure, 20(8):569-576, 2014.

[140] Gero Walter and Thomas Augustin. Bayesian linear regression-different conjugate models and their (in) sensitivity to prior-data conflict. In Kneib T., Tutz G. (eds) Statistical Modelling and Regression Structures, pages 59-78. Springer, 2010.

[141] Robert WM Wedderburn. Quasi-likelihood functions, generalized linear models, and the gaussnewton method. Biometrika, 61(3):439-447, 1974.

[142] John M Williamson, Hung Mo Lin, and Huiman X Barnhart. A classification statistic for GEE categorical response models. Journal of Data Science, 1:149_ $165,2003$.

[143] Jurgen Windeler and Stefan Lange. Events per person year-a dubious concept. BMJ: British Medical Journal, 310(6977):454, 1995.

[144] $\mathrm{H} \mathrm{Xu}$. LOCF method and application in clinical data analysis. In Poster presented at the annual meeting of the Northeast SAS Users Group (NESUG). Abstract retrieved from http://www. nesug. org/Proceedings/nesug09/po/po12. pdf, 2009.

[145] Clyde W Yancy, Mariell Jessup, Biykem Bozkurt, Javed Butler, Donald E Casey, Mark H Drazner, Gregg C Fonarow, Stephen A Geraci, Tamara Horwich, and James L Januzzi. 2013 ACCF/AHA guideline for the management of heart failure: executive summary: a report of the american college of cardiology foundation/american heart association task force on practice guidelines. Journal of the American College of Cardiology, 62(16):1495-1539, 2013. 
[146] Michihiro Yoshimura, Yuji Mizuno, Masafumi Nakayama, Tomohiro Sakamoto, Seigo Sugiyama, Hiroaki Kawano, Hirofumi Soejima, Nobutaka Hirai, Yoshihiko Saito, Kazuwa Nakao, et al. B-type natriuretic peptide as a marker of the effects of enalapril in patients with heart failure. The American Journal of Medicine, 112(9):716-720, 2002.

[147] Scott L Zeger and Kung Yee Liang. Longitudinal data analysis for discrete and continuous outcomes. Biometrics, 42(1):121-130, 1986.

[148] Scott L Zeger, Kung-Yee Liang, and Paul S Albert. Models for longitudinal data: a generalized estimating equation approach. Biometrics, 40(4):1049-1060, 1988.

[149] Jianchun Zhang, Lanju Zhang, and Harry Yang. Sample size consideration for immunoassay screening cut-point determination. Journal of Biopharmaceutical Statistics, 24(3):535-545, 2014. 


\section{Publication List}

Davarzani, N., Sanders-van Wijk S., Maeder M.T., Rickenbacher P., Smirnov E., Karel J., Suter T., de Boer R.A., Block D., Rolny V., Zaugg Ch., Pfisterer M.E., Peeters, R., Brunner-La Rocca, H.P. (2018). Novel concept to guide systolic heart failure medication by repeated biomarker testing - results from TIME-CHF in context of predictive, preventive and personalised medicine. The EPMA Journal, 2018. To appear.

Davarzani, N., West P.N., Hutchins, G.A., Hewitt C.L., Cunningham, D., Allum, H.W., Smyth, E., Valeri, N., Langley, R.E., Grabsch, I.H. (2018). Prognostic value of pathological lymph node status and primary tumour regression after neoadjuvant chemotherapy results from the MRC OE02 oesophageal cancer trial. Journal of Histopathology. 72(7): 1180-1188.

Icuma, T., Achcar, J.A., Martinez, E.Z., Davarzani, N. (2018). Determination of optimum medical cut points for continuous covariates in lifetime regression models. Journal of Model Assisted Statistics and Applications. 13(2):141-159.

Davarzani, N., Achcar, J.A., Karel, J. Smirnov, E., Brunner-La Rocca, H.P., Peeters, R. (2018). Cutpoint estimation for continuous covariates dichotomization. Submitted.

Davarzani, N., Sanders-van Wijk, S., Maeder, M., Burkart, T., Rickenbacher, P., Estlinbaum, W., Erne, P., Beer, H.J., Pfisterer, M.E., Brunner-La Rocca, H.P. (2017). NTproBNP guided therapy reduces repeated hospitalizations - results from TIME-CHF. Journal of Cardiac Failure, 23(5): 382-389.

Davarzani, N., Achcar, J.A., Peeters, R., Smirnov, E. (2017). A Bayesian analysis for the bivariate geometric distribution in the presence of covariates and censored data. Journal of Statistics and Management Systems, 20(1): 1-16.

Davarzani, N., Golparvar, L., Parsian, A. (2017). Estimation on dependent left censoring in Marshall-Olkin bivariate exponential. In revision.

Davarzani, N., Smirnov, E., Peeters, R, Karel, J., Brunner-La Rocca, H.P. (2016). Ranking Accuracy for Logistic-GEE Models. In: Boström H., Knobbe A., Soares C., Papapetrou P. (eds), Advances in Intelligent Data Analysis XV. Proceedings of IDA 2016. Lecture 
Notes in Computer Science, 9897: 14-25.

Davarzani, N., Golparvar, L., Parsian, A., Peeters, R. (2016). Estimation on dependent right censoring scheme in an ordinary bivariate geometric distribution. Journal of Applied Statistics, 44(8): 1-16.

Icuma, T.R., Buzatto, E.P., Tiezzi, D.G., Achcar, J.A., Davarzani, N. (2016). BasuDhar bivariate geometric distribution in the presence of covariates and censored data: A Bayesian approach. Journal of Data Science, 14(4): 657-680.

Sanders-van Wijk, S., Van Empel, V., Davarzani, N., Maeder, M.T., Muzzarelli, S., Jeker, U., Dieterle, T., Handschin, R., Kiencke, S., Pfisterer, ME., Brunner-La Rocca, H.P., for the TIME-CHF investigators. (2015). Circulating biomarkers of distinct pathophysiological pathways in heart failure with preserved vs. reduced left ventricular ejection fraction. European journal of heart failure, 17(10): 1006-1014.

Achcar, J.A., Davarzani, N., Souza, M.R. (2015). Basu-Dhar bivariate geometric distribution in the presence of covariates and censored data: A Bayesian approach. Journal of Applied Statistics, 43(9): 1636-1648.

Davarzani, N., Achcar, J.A., Smirnov, E., Peeters, R. (2015). Bivariate lifetime geometric distribution in presence of cure fraction. Journal of Data Science, 13(4): 755-770.

Davarzani, N., Parsian, A., Peeters, R. (2015). Dependent right censorship in the MarshallOlkin bivariate Weibull distribution. Communication in Statistics - Theory and Methods, 44(11): 2222-2242.

Davarzani, N., Parsian, A., Peeters, R. (2015). Statistical Inference on Middle-Censored Data in a Dependent Setup. Journal of Statistical Theory and Practice, 9(3): 646-657.

Davarzani, N., Parsian, A. (2013). Inference under right censoring in a discrete setup. Communication in Statistics - Theory and Methods, 42(13): 2362-2375. 


\section{Summary}

Heart failure (HF) occurs when the heart muscle does not pump sufficient blood to fulfill the requirements of the body as it should. HF is one of the most important causes of hospitalization for patients aged 65 and over, which increasingly imposes high health care costs related to relatively long hospital stays. HF is a very complex disease and it is difficult to diagnose and monitor the progression of the disease based on clinical signs and symptoms only. This is even more difficult for elderly patients with multiple comorbidities. Therefore, biomarkers (circulating markers that can be measured in the blood) that reflect one or several pathways might be useful as diagnostic or prognostic factors in HF. Biomarkers may also have therapeutic implications for individualized therapy. Guiding HF therapy by a single biomarker, measured only at one time point, is limited because one biomarker at one time point cannot cover the extensive pathophysiological pathways involved in HF. Performing repeated measurements of several biomarkers, however, may lead towards precision in medical therapy in HF patients.

This thesis had two main objectives; namely a medical objective and a methodological one. The medical objective of this thesis was to investigate the predictive value of biomarkers to predict the response to therapy in the Trial of Intensified versus standard Medical therapy in Elderly patients with Congestive Heart Failure (TIME-CHF) study. The methodological objective of the thesis was to develop new methods with the aim to be used for addressing the medical objective and to facilitate investigation of biomarkers.

In Chapter 2, we provided general background information about the TIME-CHF study and the statistical methods applied in this thesis.

In Chapter 3, we employed the Prentice, Williams and Peterson Gap-Times model $[10,106]$ to investigate the effect of N-terminal pro-B-type Natriuretic Peptide (NTproBNP)-guided therapy on the recurrence of all-cause hospitalization events (all-cause hospitalization or death) and HF hospitalization events (HF hospitalization or death) in the TIME-CHF study. We observed that the NT-proBNP-guided therapy as compared to standard therapy did not reduce the risk of first all-cause hospitalization event. However, we showed that NT-proBNP-guided therapy has a beneficial effect on the second and third all-cause hospitalization events, indicating that considering repeated events may be meaningful in interventional clinical trials. When considering pre-stratified age groups, these effects were only seen in patients aged $<75$ years, but not in patients aged $\geq 75$ years. In addition, NT-proBNP-guided therapy reduced the risk of first and second HF hospitalization again predominantly in patients aged $<75$ years.

In this thesis we aimed to move towards developing a biomarker-guided treatment 
algorithm for personalized medical HFrEF (HF with reduced Ejection Fraction) therapy (Chapter 6). As a purely hypothesis generating study, we investigated the interaction between multiple repeatedly measured biomarkers and the response to the four most important classes of HF medication regarding the risk of HF hospitalization or death in patients with reduced ejection fraction. For these analyses, we employed logistic Generalized Estimating Equations (logistic-GEE) model [73, 147].

The four HF medications in this study were $\beta$-blockers, Renin-Angiotensin System (RAS) blockers, Spironolactone and Loop diuretics. The study also included the following briomarkers: Soluble FMS-Like Tyrosine kinase-1 (SFLT), Growth Differentiation Factor 15, Cystatin-C (CysC), Ferritin, Interleukin-6 (IL6), Placental Like Growth Factor, Sex Hormone-Binding Globulin, Soluble TransFerrin Receptor, High Sensitivity Troponin T, Type-1 Procollagen N-terminal Peptide, Uric acid, Blood Urea Nitrogen (BUN), Soluble ST2 (SST2), NT-proBNP, Creatinine, High Sensitivity C-Reactive Protein (HSCRP), Prealbumin (PREA), Osteopontin, Mimican, and Insulin-like Growth Factor-Binding Protein 7.

The results showed that loop diuretics up-titration has a beneficial effect for patients with high IL6 or high HSCRP, whereas the opposite was true with low IL6 or low HSCRP. Higher dosage of loop diuretics was associated with poor outcome in patients with high BUN or PREA, but not in those with low levels of these biomarkers. Spironolactone uptitration was associated with lower risk of HF hospitalization or death in patients with high CysC. Patients with a high SFLT concentration may have a worse outcome with higher doses of $\beta$-blockers as compared to those on lower doses. Patients with low SFLT levels had a more favourable outcome overall, largely irrespective of $\beta$-blockers dose in our population. No treatment biomarker interactions were found for RAS blockers. Such interactions between biomarkers and treatment might help to personalize treatment in HFrEF patients, but prospective validation is required before this approach can be applied to clinical practice. In order to support and assess the accuracy of these results, in Chapter 5 , we proposed a new performance measure for logistic-GEE models, which we called Generalized Ranking Accuracy (GRA).

GRA is defined as the probability that a randomly selected instance with positive outcome is ranked higher than a randomly selected instance with negative outcome from another cluster, with logistic-GEE model. There are several advantages with GRA compared with other standard goodness-of-fit criteria for logistic-GEE models: (a) there is no restriction in computation of GRA regarding the number and types of covariates in the model; (b) GRA as a probability is easy to interpret; (c) GRA has an absolute range and can be used for comparing the goodness-of-fit of different logistic-GEE models for different data sets; and (d) by using cross-validation, GRA-CV can be used as a predictive performance criterion for logistic-GEE models. In addition we showed experimentally that GRA is not biased in the presence of two-class imbalanced data. Moreover, we showed 
that GRA-CV performs well as a model selection criterion for logistic-GEE models. We note that GRA is also applicable to any model for bipartite ranking trained on clustered data.

Dichotomizing the biomarkers (or any continuous covariates) may make it easier to interpret the obtained statistical results and may help to have a more precise treatment decision. Therefore, we introduced a Bayesian approach (Chapter 4) to estimate cutpoints for continuous covariates. The proposed approach is based on the assumption that the dichotomized covariates are related to an outcome variable through linear regression. In the multivariate setting, this approach can be used to dichotomize all or a subset of the covariates. To the best of our knowledge the proposed Bayesian approach is the first method that can deal with multivariate dichotomization with respect to modeling a continuous outcome variable. We applied the Bayesian approach to estimate the cutpoints for three markers in the TIME-CHF study, the BMI (Body Mass Index) and two biomarkers: SST2 and Mimecan, with respect to NT-proBNP. We observed that the estimated cutpoints and associations of the dichotomized markers with NT-proBNP were in the same line with the recommended cutpoints and previously observed associations in the other studies. 


\section{Valorization}

Heart Failure (HF) is recognized as an escalating public health problem in Western societies with ageing populations [51, 83]. In addition, HF is the most common reason for hospitalization in patients aged 65 years and over which imposes enormous health care costs related to relatively long hospital stays [81, 127]. Thus, HF accounts for almost $2 \%$ of health care budget in western countries and its total economic burden is increasing, the greatest portions attributable to HF related hospitalizations. These health care costs can be decreased by having a more precise diagnosis of disease in HF patients and implementing more effective therapy. Diagnosing HF and treating patients on clinical signs and symptoms can be challenging, as signs and symptoms of HF are not specific. Therefore, biomarkers might be helpful in this regard. The obtained results in this thesis improve implementation of biomarkers in diagnosis of $\mathrm{HF}$ and guiding individualized medical HF therapy. In addition, we developed new methods with the aim to facilitate investigation of biomarkers. In this thesis the analysis were based on the Trial of Intensified versus standard Medical therapy in Elderly patients with Congestive Heart Failure (TIME-CHF) study $[19,102]$. In this chapter we provide valorization opportunities result from this thesis, which may be of interest for medical researchers, patients, clinicians and pharmaceutical companies.

Although HF patients are known to experience repeated hospitalizations, most studies only evaluated the time-to-first event and do not consider the impact of therapy after the initial event. The results in this thesis demonstrated that guiding the therapy based on N-terminal pro-B-type Natriuretic Peptide (NT-proBNP) versus standard symptoms reduces the risk of recurrent hospitalization in HF patients (Chapter 3). This leads to the decrease in hospitalization costs for the health system. Even more importantly, medical intervention studies usually use time-to-first event methods only. Our result show that these methods may result in the wrong conclusions.

The results in Chapter 6 suggest that repeated measurements of biomarkers might be helpful to individually tailor HF treatment to optimize the balance between beneficial and adverse effects of HF drugs in a particular patient. This might also be economically beneficial, since patients would have better outcome (less hospitalizations, less side effects) with less medication, thereby reducing costs. However, this novel predictive, preventive and personalized medicine approach clearly needs confirmation in other studies.

Dichotomizing continuous covariates is widespread in medical prognostic variable studies. In Chapter 4, we proposed a Bayesian approach to dichotomize continuous covariates. This might help clinicians to have a more precise interpretation of the covariate's 
effect on the outcome variable and guides them in their choice of therapy. In addition, it can establish eligibility criteria for prospective studies. Moreover, some researchers prefer using dichotomized covariates since they are more straightforward to analyze and the interpretation of the results may be easier.

In Chapter 5, we proposed Generalized Ranking Accuracy (GRA) as a measure for assessing the goodness-of-fit as well as the predictive performance of the models trained on clustered data. Clustered data are very common in biomedical and clinical researches when the observations for each subject are repeated over time. Using GRA for models trained on the clinical cluster data may help the researchers in their result interpretations and help clinicians in making an appropriate treatment decision. 


\section{Acknowledgements}

My gratitude goes to my supervisors Prof. Hans-Peter Brunner-La Rocca and Prof. Ralf Peeters for guiding me throughout this research and for all productive discussions we had. My sincere appreciation goes to my co-supervisors Dr. Evgueni Smirnov and Dr. Joël Karel for their endless support and help. I would like to thank Dr. Sandra Sanders-van Wijk for her great help regarding the background on cardiology.

I would like to express my gratitude to Prof. Heike Grabsch who provided me the opportunity to work at the Department of Pathology. I have learned a lot from her about Pathology. I would like to thank her for the supervision and support she consistently offered in the last two years. I would like to thank Prof. Ernst-Jan Speel for welcoming me and giving me the chance of working in his group.

Prof. Ahmad Parsian, I am so grateful for all your supports. Due to you, I have not only advanced a lot in Statistics, but also learned more about Morality. Thank you for being always beside me in this journey. I would like to thank Prof. Jorge Alberto Achcar for the collaborations we had during my $\mathrm{PhD}$.

I would like to express my gratitude to the members of assessment committee who devoted their time to read my thesis and provided valuable feedback for future improvement. Prof. Gerhard Weiss, Prof. Ilja Arts, Prof. Geert Molenberghs, Prof. Christian E. Müller and Dr. Vanessa van Empel, it is my honor to get the approval on my thesis from you.

I would like to thank my DKE officemates, Firat Ismailoglu, Shuang Zhou, Chiara Sironi, Hua Zhang, Arjun Krishnamurthy and Yiyong Gou, thanks for making our office a great place to work. I would like to thank my colleagues in Maastricht University at Department of Knowledge Engineering, Department of Cardiology and Department of Pathology, Ronald Westra, Jet Custers, Pietro Bonizzi, Rachel Cavill, Katerina Stankova, Frank Thuijsman, Gijs Schoenmakers, Nico Roos, Wei Zhao, Li You, Katharina Schüller, Matthijs Cluitmans, Esan Ghaleb, Christos Athanasiadis, Dario Dotti, Amir Ahangi, Maryam Karimian, Lindsay Hewitt, Gregorio Fazzi, Han Jin, Erwin Wijnands, Clairy Dinjens, Muriel Draht, Zheng Feng, Glenn Rademakers, Nathalie Vaes, Jaleesa van der Meer, Alexander koch, Kim Wouters, Danielle Vanderschuren.

I would like to thank my parents and lovely siblings Yaser, Azadeh and Mahdi. I would like to express my heartfelt gratitude to my uncle Ghasem Pourpak for all his supports.

My especial thanks goes to Sepideh who has been always beside me in all moments, whether hard or happy. 
Thanks to my dear Maastricht family, Iman, Ortrun, Irina, Dorcas, Ibrahima and Shuan for sharing all good moments with me. Iman, I owe you so much. You are the one always being supportive. I cannot imagine what would have been my situation without your help. Ortrun I will never forget the trip we had in Munich and your lovely mom's hosting. Irina and Dorcas, you are the best companies and hard to find. We had many happy moments here in Maastricht and Amsterdam, our special brunch, dinner and movie events. I am so proud of our friendship. Ibrahima, you are my Mr. President. I really appreciate your talents in politics, dance and singing. I am dreaming a day to see you one top of the politics mountain. I will never forget all the happy moments we had in Maastricht. Shuan, you are the one who is always available in the hard moments with lot of approaches. I have learned a lot from you, especially about the Japanese culture.

Maryam Salehijam, thank you for all your supports even remotely when you have left Maastricht. Alex Mohajer you are the one that I always love to listen to his advises. I have learned a lot about the life from you. Sir Sohrab and Khale Mitra, thank you very much for all the gatherings you organized and all happy moments you have shared with us.

Ahmah Salahati Ghalejooghi, thank you very much for all the Rahnamoods you were sharing with me over the lunch time. I have shared lots of energy and somehow happy moments with you.

Mehrdad, thanks a lot for all gathering and events you organized. I always enjoy spending time with you and Sanne. My lovely couples, Nastaran and Ehsan, I always enjoy companying you. You are wonderful friends and fellow travellers. Saba jan, you are a great friend and specially a good advisor. Sorry if I have never followed any of your advises. Lumiere and I are going to miss you. Iman and Julia you were the great neighbours. Thanks to Arash for all his supports. Meshkat, you are a good friend and a great doctor with your magical healing drops. Sofya jan, thanks a lot for all workshops and goodbye parties you organized. Hoda, Bijan and Hossein we have started life in Maastricht together and we have shared lots of happy moments. Somaieh jan, thanks for the biking tours you were organizing. Ali Gharaviri, you are a great friend. I had great time in Maastricht with you. Ehsan Ramezanifar I always enjoyed spending time with you. Roya and Hasti I am so happy you moved to Maastricht.

Thanks to my lovely friends from my bachelor studies: Ezam, Rasoul, Gelareh, Sara, Neda, Alireza, Elham, Vahid and Mahdieh for all their supports. My especial thanks goes to Nasser Rad, the man under the shadow, for all his helps and supports.

Thanks to all my friends in Maastricht that shared happy moments with me, Kiyana, Eliyeh, Sirous, Negar, Taniya, Linda, Sebastian, Rahi, Alisom, Elisa, Kristina, Olga, Masoud, Mehrdad and Parizad. 


\section{About the Author}

Naser Davarzani was born on June 20, 1983 in Tehran, Iran. He graduated from Allameh Tabataba'i University with B.Sc. in Statistics and from Payame Noor University with MSc. in Mathematical Statistics. His Master's thesis focused on Bayesian analysis in censored data and its results were published as three papers. In March 2012, he moved to Maastricht University to conduct his $\mathrm{PhD}$ at Department of Data Science and Knowledge Engineering in partnership with Department of Cardiology, Maastricht University Medical Centre. His PhD was part of a research study, TIMECHF, in collaboration with Roche. During his $\mathrm{PhD}$ research, his scientific contributions resulted in various high-level conference and journal publications. Since December 2015, he

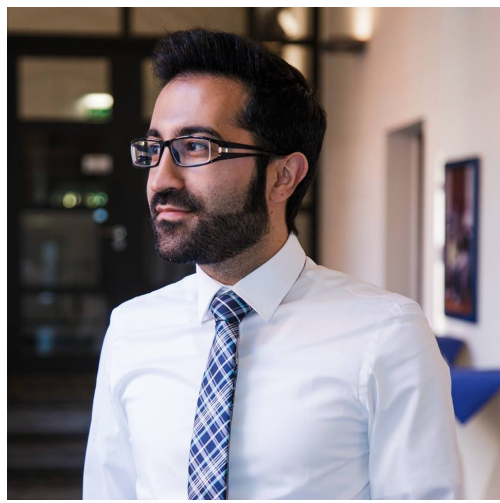
has been working as a Biostatistician at Department of Pathology, GROW School for Oncology and Developmental Biology, Maastricht University, on the topic of gastric cancer. 


\section{SIKS Dissertation Series}

\section{1}

1. Botond Cseke (RUN), Variational Algorithms for Bayesian Inference in Latent Gaussian Models.

2. Nick Tinnemeier (UU), Organizing Agent Organizations. Syntax and Operational Semantics of an Organization-Oriented Programming Language.

3. Jan Martijn van der Werf (TUE), Compositional Design and Verification of Component-Based Information Systems.

4. Hado van Hasselt (UU), Insights in Reinforcement Learning; Formal analysis and empirical evaluation of temporal-difference.

5. Bas van der Raadt (VU), Enterprise Architecture Coming of Age - Increasing the Performance of an Emerging Discipline.

6. Yiwen Wang (TUE), SemanticallyEnhanced Recommendations in Cultural Heritage.

7. Yujia Cao (UT), Multimodal Information Presentation for High Load Human Computer Interaction.

8. Nieske Vergunst (UU), BDI-based Generation of Robust Task-Oriented Dialogues.
9. Tim de Jong (OU), Contextualised Mobile Media for Learning.

10. Bart Bogaert (UvT), Cloud Content Contention.

11. Dhaval Vyas (UT), Designing for Awareness: An Experience-focused HCI Perspective.

12. Carmen Bratosin (TUE), Grid Architecture for Distributed Process Mining.

13. Xiaoyu Mao (UvT), Airport under Control. Multiagent Scheduling for Airport Ground Handling.

14. Milan Lovric (EUR), Behavioral Finance and Agent-Based Artificial Markets.

15. Marijn Koolen (UvA), The Meaning of Structure: the Value of Link Evidence for Information Retrieval.

16. Maarten Schadd (UM), Selective Search in Games of Different Complexity.

17. Jiyin He (UVA), Exploring Topic Structure: Coherence, Diversity and Relatedness.

18. Mark Ponsen (UM), Strategic Decision-Making in complex games.

19. Ellen Rusman (OU), The Mind's Eye on Personal Profiles. 
20. Qing $\mathrm{Gu}$ (VU), Guiding serviceoriented software engineering - A view-based approach.

21. Linda Terlouw (TUD), Modularization and Specification of ServiceOriented Systems.

22. Junte Zhang (UVA), System Evaluation of Archival Description and Access.

23. Wouter Weerkamp (UVA), Finding People and their Utterances in Social Media.

24. Herwin van Welbergen (UT), Behavior Generation for Interpersonal Coordination with Virtual Humans On Specifying, Scheduling and Realizing Multimodal Virtual Human Behavior.

25. Syed Waqar ul Qounain Jaffry (VU), Analysis and Validation of Models for Trust Dynamics.

26. Matthijs Aart Pontier (VU), Virtual Agents for Human Communication - Emotion Regulation and Involvement-Distance Trade-Offs in Embodied Conversational Agents and Robots.

27. Aniel Bhulai (VU), Dynamic website optimization through autonomous management of design patterns.

28. Rianne Kaptein (UVA), Effective Focused Retrieval by Exploiting Query Context and Document Structure.

29. Faisal Kamiran (TUE), Discriminationaware Classification.
30. Egon van den Broek (UT), Affective Signal Processing (ASP): Unraveling the mystery of emotions.

31. Ludo Waltman (EUR), Computational and Game-Theoretic Approaches for Modeling Bounded Rationality.

32. Nees-Jan van Eck (EUR), Methodological Advances in Bibliometric Mapping of Science.

33. Tom van der Weide (UU), Arguing to Motivate Decisions.

34. Paolo Turrini (UU), Strategic Reasoning in Interdependence: Logical and Game-theoretical Investigations.

35. Maaike Harbers (UU), Explaining Agent Behavior in Virtual Training.

36. Erik van der Spek (UU), Experiments in serious game design: a cognitive approach.

37. Adriana Burlutiu (RUN), Machine Learning for Pairwise Data, Applications for Preference Learning and Supervised Network Inference.

38. Nyree Lemmens (UM), Bee-inspired Distributed Optimization.

39. Joost Westra (UU), Organizing Adaptation using Agents in Serious Games.

40. Viktor Clerc (VU), Architectural Knowledge Management in Global Software Development. 
41. Luan Ibraimi (UT), Cryptographically Enforced Distributed Data Access Control.

42. Michal Sindlar (UU), Explaining Behavior through Mental State Attribution.

43. Henk van der Schuur (UU), Process Improvement through Software Operation Knowledge.

44. Boris Reuderink (UT), Robust BrainComputer Interfaces.

45. Herman Stehouwer (UvT), Statistical Language Models for Alternative Sequence Selection.

46. Beibei Hu (TUD), Towards Contextualized Information Delivery: A Rule-based Architecture for the Domain of Mobile Police Work.

47. Azizi Bin Ab Aziz (VU), Exploring Computational Models for Intelligent Support of Persons with Depression.

48. Mark Ter Maat (UT), Response Selection and Turn-taking for a Sensitive Artificial Listening Agent.

49. Andreea Niculescu (UT), Conversational interfaces for task-oriented spoken dialogues: design aspects influencing interaction quality.

\section{2}

1. Terry Kakeeto (UvT), Relationship Marketing for SMEs in Uganda.
2. Muhammad Umair (VU), Adaptivity, emotion, and Rationality in Human and Ambient Agent Models.

3. Adam Vanya (VU), Supporting Architecture Evolution by Mining Software Repositories.

4. Jurriaan Souer (UU), Development of Content Management Systembased Web Applications.

5. Marijn Plomp (UU), Maturing Inter organisational Information Systems.

6. Wolfgang Reinhardt (OU), Awareness Support for Knowledge Workers in Research Networks.

7. Rianne van Lambalgen (VU), When the Going Gets Tough: Exploring Agent-based Models of Human Performance under Demanding Conditions.

8. Gerben de Vries (UVA), Kernel Methods for Vessel Trajectories.

9. Ricardo Neisse (UT), Trust and Privacy Management Support for Context-Aware Service Platforms.

10. David Smits (TUE), Towards a Generic Distributed Adaptive Hypermedia Environment.

11. J.C.B. Rantham Prabhakara (TUE), Process Mining in the Large: Preprocessing, Discovery, and Diagnostics.

12. Kees van der Sluijs (TUE), Model Driven Design and Data Integration in Semantic Web Information Systems. 
13. Suleman Shahid (UvT), Fun and Face: Exploring non-verbal expressions of emotion during playful interactions.

14. Evgeny Knutov (TUE), Generic Adaptation Framework for Unifying Adaptive Web-based Systems.

15. Natalie van der Wal (VU), Social Agents. Agent-Based Modelling of Integrated Internal and Social Dynamics of Cognitive and Affective Processes.

16. Fiemke Both (VU), Helping people by understanding them - Ambient Agents supporting task execution and depression treatment.

17. Amal Elgammal (UvT), Towards a Comprehensive Framework for Business Process Compliance.

18. Eltjo Poort (VU), Improving Solution Architecting Practices.

19. Helen Schonenberg (TUE), What's Next? Operational Support for Business Process Execution.

20. Ali Bahramisharif (RUN), Covert Visual Spatial Attention, a Robust Paradigm for Brain-Computer Interfacing.

21. Roberto Cornacchia (TUD), Querying Sparse Matrices for Information Retrieval.

22. Thijs Vis (UvT), Intelligence, politie en veiligheidsdienst: verenigbare grootheden?
23. Christian Muehl (UT), Toward Affective Brain-Computer Interfaces: Exploring the Neurophysiology of Affect during Human Media Interaction.

24. Laurens van der Werff (UT), Evaluation of Noisy Transcripts for Spoken Document Retrieval.

25. Silja Eckartz (UT), Managing the Business Case Development in InterOrganizational IT Projects: A Methodology and its Application.

26. Emile de Maat (UVA), Making Sense of Legal Text.

27. Hayrettin Gurkok (UT), Mind the Sheep! User Experience Evaluation \& Brain-Computer Interface Games.

28. Nancy Pascall (UvT), Engendering Technology Empowering Women.

29. Almer Tigelaar (UT), Peer-to-Peer Information Retrieval.

30. Alina Pommeranz (TUD), Designing Human-Centered Systems for Reflective Decision Making.

31. Emily Bagarukayo (RUN), A Learning by Construction Approach for Higher Order Cognitive Skills Improvement, Building Capacity and Infrastructure.

32. Wietske Visser (TUD), Qualitative multi-criteria preference representation and reasoning.

33. Rory Sie (OUN), Coalitions in Cooperation Networks (COCOON). 
34. Pavol Jancura (RUN), Evolutionary analysis in PPI networks and applications.

35. Evert Haasdijk (VU), Never Too Old To Learn - On-line Evolution of Controllers in Swarm- and Modular Robotics.

36. Denis Ssebugwawo (RUN), Analysis and Evaluation of Collaborative Modeling Processes.

37. Agnes Nakakawa (RUN), A Collaboration Process for Enterprise Architecture Creation.

38. Selmar Smit (VU), Parameter Tuning and Scientific Testing in Evolutionary Algorithms.

39. Hassan Fatemi (UT), Risk-aware design of value and coordination networks.

40. Agus Gunawan (UvT), Information Access for SMEs in Indonesia.

41. Sebastian Kelle (OU), Game Design Patterns for Learning.

42. Dominique Verpoorten (OU), Reflection Amplifiers in self-regulated Learning.

43. Withdrawn

44. Anna Tordai (VU), On Combining Alignment Techniques.

45. Benedikt Kratz (UvT), A Model and Language for Business-aware Transactions.
46. Simon Carter (UVA), Exploration and Exploitation of Multilingual Data for Statistical Machine Translation.

47. Manos Tsagkias (UVA), Mining Social Media: Tracking Content and Predicting Behavior.

48. Jorn Bakker (TUE), Handling Abrupt Changes in Evolving Timeseries Data.

49. Michael Kaisers (UM), Learning against Learning - Evolutionary dynamics of reinforcement learning algorithms in strategic interactions.

50. Steven van Kervel (TUD), Ontologogy driven Enterprise Information Systems Engineering.

51. Jeroen de Jong (TUD), Heuristics in Dynamic Sceduling; a practical framework with a case study in elevator dispatching.

\section{3}

1. Viorel Milea (EUR), News Analytics for Financial Decision Support.

2. Erietta Liarou (CWI), MonetDB/DataCell: Leveraging the Column-store Database Technology for Efficient and Scalable Stream Processing.

3. Szymon Klarman (VU), Reasoning with Contexts in Description Logics.

4. Chetan Yadati (TUD), Coordinating autonomous planning and scheduling. 
5. Dulce Pumareja (UT), Groupware Requirements Evolutions Patterns.

6. Romulo Goncalves (CWI), The Data Cyclotron: Juggling Data and Queries for a Data Warehouse Audience.

7. Giel van Lankveld (UvT), Quantifying Individual Player Differences.

8. Robbert-Jan Merk (VU), Making enemies: cognitive modeling for opponent agents in fighter pilot simulators.

9. Fabio Gori (RUN), Metagenomic Data Analysis: Computational Methods and Applications.

10. Jeewanie Jayasinghe Arachchige (UvT), A Unified Modeling Framework for Service Design.

11. Evangelos Pournaras (TUD), Multi-level Reconfigurable Selforganization in Overlay Services.

12. Marian Razavian (VU), Knowledgedriven Migration to Services.

13. Mohammad Safiri (UT), Service Tailoring: User-centric creation of integrated IT-based home care services to support independent living of elderly.

14. Jafar Tanha (UVA), Ensemble Approaches to Semi-Supervised Learning.

15. Daniel Hennes (UM), Multiagent Learning - Dynamic Games and Applications.
16. Eric Kok (UU), Exploring the practical benefits of argumentation in multi-agent deliberation.

17. Koen Kok (VU), The Power Matcher: Smart Coordination for the Smart Electricity Grid.

18. Jeroen Janssens (UvT), Outlier Selection and One-Class Classification.

19. Renze Steenhuizen (TUD), Coordinated Multi-Agent Planning and Scheduling.

20. Katja Hofmann (UvA), Fast and Reliable Online Learning to Rank for Information Retrieval.

21. Sander Wubben (UvT), Text-to-text generation by monolingual machine translation.

22. Tom Claassen (RUN), Causal Discovery and Logic.

23. Patricio de Alencar Silva (UvT), Value Activity Monitoring.

24. Haitham Bou Ammar (UM), Automated Transfer in Reinforcement Learning.

25. Agnieszka Anna LatoszekBerendsen (UM), Intention-based Decision Support. A new way of representing and implementing clinical guidelines in a Decision Support System.

26. Alireza Zarghami (UT), Architectural Support for Dynamic Home care Service Provisioning. 
27. Mohammad Huq (UT), Inferencebased Framework Managing Data Provenance.

28. Frans van der Sluis (UT), When Complexity becomes Interesting: An Inquiry into the Information experience

29. Iwan de Kok (UT), Listening Heads

30. Joyce Nakatumba (TUE), ResourceAware Business Process Management: Analysis and Support

31. Dinh Khoa Nguyen (UvT), Blueprint Model and Language for Engineering Cloud Applications

32. Kamakshi Rajagopal (OUN), Networking For Learning; The role of Networking in a Lifelong Learner's Professional Development

33. Qi Gao (TUD), User Modeling and Personalization in the Microblogging Sphere.

34. Kien Tjin-Kam-Jet (UT), Distributed Deep Web Search.

35. Abdallah El Ali (UvA), Minimal Mobile Human Computer Interaction.

36. Than Lam Hoang (TUe), Pattern Mining in Data Streams.

37. Dirk Börner (OUN), Ambient Learning Displays.

38. Eelco den Heijer (VU), Autonomous Evolutionary Art.
39. Joop de Jong (TUD), A Method for Enterprise Ontology based Design of Enterprise Information Systems.

40. Pim Nijssen (UM), Monte-Carlo Tree Search for Multi-Player Games.

41. Jochem Liem (UVA), Supporting the Conceptual Modelling of Dynamic Systems: A Knowledge Engineering Perspective on Qualitative Reasoning.

42. Léon Planken (TUD), Algorithms for Simple Temporal Reasoning.

43. Marc Bron (UVA), Exploration and Contextualization through Interaction and Concepts.

\section{4}

1. Nicola Barile (UU), Studies in Learning Monotone Models from Data.

2. Fiona Tuliyano (RUN), Combining System Dynamics with a Domain Modeling Method.

3. Sergio Raul Duarte Torres (UT), Information Retrieval for Children: Search Behavior and Solutions.

4. Hanna Jochmann-Mannak (UT), Websites for children: search strategies and interface design Three studies on children's search performance and evaluation.

5. Jurriaan van Reijsen (UU), Knowledge Perspectives on Advancing Dynamic Capability. 
6. Damian Tamburri (VU), Supporting Networked Software Development.

7. Arya Adriansyah (TUE), Aligning Observed and Modeled Behavior.

8. Samur Araujo (TUD), Data Integration over Distributed and Heterogeneous Data Endpoints.

9. Philip Jackson (UvT), Toward Human-Level Artificial Intelligence: Representation and Computation of Meaning in Natural Language.

10. Ivan Salvador Razo Zapata (VU), Service Value Networks.

11. Janneke van der Zwaan (TUD), An Empathic Virtual Buddy for Social Support.

12. Willem van Willigen (VU), Look Ma, No Hands: Aspects of Autonomous Vehicle Control.

13. Arlette van Wissen (VU), AgentBased Support for Behavior Change: Models and Applications in Health and Safety Domains.

14. Yangyang Shi (TUD), Language Models With Meta-information.

15. Natalya Mogles (VU), Agent-Based Analysis and Support of Human Functioning in Complex SocioTechnical Systems: Applications in Safety and Health care.

16. Krystyna Milian (VU), Supporting trial recruitment and design by automatically interpreting eligibility criteria.
17. Kathrin Dentler (VU), Computing health care quality indicators automatically: Secondary Use of Patient Data and Semantic Interoperability

18. Mattijs Ghijsen (UVA), Methods and Models for the Design and Study of Dynamic Agent Organizations.

19. Vinicius Ramos (TUE), Adaptive Hypermedia Courses: Qualitative and Quantitative Evaluation and Tool Support.

20. Mena Habib (UT), Named Entity Extraction and Disambiguation for Informal Text: The Missing Link.

21. Kassidy Clark (TUD), Negotiation and Monitoring in Open Environments.

22. Marieke Peeters (UU), Personalized Educational Games - Developing agent-supported scenario-based training.

23. Eleftherios Sidirourgos (UvA/CWI), Space Efficient Indexes for the Big Data Era.

24. Davide Ceolin (VU), Trusting Semistructured Web Data.

25. Martijn Lappenschaar (RUN), New network models for the analysis of disease interaction.

26. Tim Baarslag (TUD), What to Bid and When to Stop.

27. Rui Jorge Almeida (EUR), Conditional Density Models Integrating 
Fuzzy and Probabilistic Representations of Uncertainty.

28. Anna Chmielowiec (VU), Decentralized k-Clique Matching.

29. Jaap Kabbedijk (UU), Variability in Multi-Tenant Enterprise Software.

30. Peter de Cock (UvT), Anticipating Criminal Behaviour.

31. Leo van Moergestel (UU), Agent Technology in Agile Multiparallel Manufacturing and Product Support.

32. Naser Ayat (UvA), On Entity Resolution in Probabilistic Data.

33. Tesfa Tegegne (RUN), Service Discovery in eHealth.

34. Christina Manteli (VU), The Effect of Governance in Global Software Development: Analyzing Transactive Memory Systems.

35. Joost van Ooijen (UU), Cognitive Agents in Virtual Worlds: A Middleware Design Approach.

36. Joos Buijs (TUE), Flexible Evolutionary Algorithms for Mining Structured Process Models.

37. Maral Dadvar (UT), Experts and Machines United Against Cyberbullying.

38. Danny Plass-Oude Bos (UT), Making brain-computer interfaces better: improving usability through postprocessing.
39. Jasmina Maric (UvT), Web Communities, Immigration, and Social Capital.

40. Walter Omona (RUN), A Framework for Knowledge Management Using ICT in Higher Education.

41. Frederic Hogenboom (EUR), Automated Detection of Financial Events in News Text.

42. Carsten Eijckhof (CWI/TUD), Contextual Multidimensional Relevance Models.

43. Kevin Vlaanderen (UU), Supporting Process Improvement using Method Increments.

44. Paulien Meesters (UvT), Intelligent Blauw. Met als ondertitel: Intelligence-gestuurde politiezorg in gebiedsgebonden eenheden.

45. Birgit Schmitz (OUN), Mobile Games for Learning: A PatternBased Approach.

46. Ke Tao (TUD), Social Web Data Analytics: Relevance, Redundancy, Diversity.

47. Shangsong Liang (UVA), Fusion and Diversification in Information Retrieval.

\section{5}

1. Niels Netten (UvA), Machine Learning for Relevance of Information in Crisis Response. 
2. Faiza Bukhsh (UvT), Smart auditing: Innovative Compliance Checking in Customs Controls.

3. Twan van Laarhoven (RUN), Machine learning for network data.

4. Howard Spoelstra (OUN), Collaborations in Open Learning Environments.

5. Christoph Bösch (UT), Cryptographically Enforced Search Pattern Hiding.

6. Farideh Heidari (TUD), Business Process Quality Computation - Computing Non-Functional Requirements to Improve Business Processes.

7. Maria-Hendrike Peetz (UvA), TimeAware Online Reputation Analysis.

8. Jie Jiang (TUD), Organizational Compliance: An agent-based model for designing and evaluating organizational interactions.

9. Randy Klaassen (UT), HCI Perspectives on Behavior Change Support Systems.

10. Henry Hermans (OUN), OpenU: design of an integrated system to support lifelong learning.

11. Yongming Luo (TUE), Designing algorithms for big graph datasets: A study of computing bisimulation and joins.
12. Julie M. Birkholz (VU), Modi Operandi of Social Network Dynamics: The Effect of Context on Scientific Collaboration Networks.

13. Giuseppe Procaccianti (VU), Energy-Efficient Software.

14. Bart van Straalen (UT), A cognitive approach to modeling bad news conversations.

15. Klaas Andries de Graaf (VU), Ontology-based Software Architecture Documentation.

16. Changyun Wei (UT), Cognitive Coordination for Cooperative MultiRobot Teamwork.

17. André van Cleeff (UT), Physical and Digital Security Mechanisms: Properties, Combinations and Trade-offs.

18. Holger Pirk (CWI), Waste Not, Want Not! - Managing Relational Data in Asymmetric Memories.

19. Bernardo Tabuenca (OUN), Ubiquitous Technology for Lifelong Learners.

20. Lois Vanhée (UU), Using Culture and Values to Support Flexible Coordination.

21. Sibren Fetter (OUN), Using PeerSupport to Expand and Stabilize Online Learning.

22. Zhemin Zhu (UT), Co-occurrence Rate Networks. 
23. Luit Gazendam (VU), Cataloguer Support in Cultural Heritage.

24. Richard Berendsen (UVA), Finding People, Papers, and Posts: Vertical Search Algorithms and Evaluation.

25. Steven Woudenberg (UU), Bayesian Tools for Early Disease Detection.

26. Alexander Hogenboom (EUR), Sentiment Analysis of Text Guided by Semantics and Structure.

27. Sándor Héman (CWI), Updating compressed colomn stores.

28. Janet Bagorogoza (TiU), Knowledge Management and High Performance; The Uganda Financial Institutions Model for HPO.

29. Hendrik Baier (UM), Monte-Carlo Tree Search Enhancements for OnePlayer and Two-Player Domains.

30. Kiavash Bahreini (OU), Real-time Multimodal Emotion Recognition in E-Learning.

31. Yakup Koç (TUD), On the robustness of Power Grids.

32. Jerome Gard (UL), Corporate Venture Management in SMEs.

33. Frederik Schadd (TUD), Ontology Mapping with Auxiliary Resources.

34. Victor de Graaf (UT), Gesocial Recommender Systems.

35. Jungxao Xu (TUD), Affective Body Language of Humanoid Robots: Perception and Effects in Human Robot
Interaction.

\section{6}

1. Syed Saiden Abbas (RUN), Recognition of Shapes by Humans and Machines.

2. Michiel Christiaan Meulendijk (UU), Optimizing medication reviews through decision support: prescribing a better pill to swallow.

3. Maya Sappelli (RUN), Knowledge Work in Context: User Centered Knowledge Worker Support.

4. Laurens Rietveld (VU), Publishing and Consuming Linked Data.

5. Evgeny Sherkhonov (UVA), Expanded Acyclic Queries: Containment and an Application in Explaining Missing Answers.

6. Michel Wilson (TUD), Robust scheduling in an uncertain environment.

7. Jeroen de Man (VU), Measuring and modeling negative emotions for virtual training.

8. Matje van de Camp (TiU), A Link to the Past: Constructing Historical Social Networks from Unstructured Data.

9. Archana Nottamkandath (VU), Trusting Crowdsourced Information on Cultural Artefacts. 
10. George Karafotias (VUA), Parameter Control for Evolutionary Algorithms.

11. Anne Schuth (UVA), Search Engines that Learn from Their Users.

12. Max Knobbout (UU), Logics for Modelling and Verifying Normative Multi-Agent Systems.

13. Nana Baah Gyan (VU), The Web, Speech Technologies and Rural Development in West Africa - An ICT4D Approach.

14. Ravi Khadka (UU), Revisiting Legacy Software System Modernization.

15. Steffen Michels (RUN), Hybrid Probabilistic Logics - Theoretical Aspects, Algorithms and Experiments.

16. Guangliang Li (UVA), Socially Intelligent Autonomous Agents that Learn from Human Reward.

17. Berend Weel (VU), Towards Embodied Evolution of Robot Organisms.

18. Albert Meroño Peñuela (VU), Refining Statistical Data on the Web.

19. Julia Efremova (Tu/e), Mining Social Structures from Genealogical Data.

20. Daan Odijk (UVA), Context \& Semantics in News \& Web Search.

21. Alejandro Moreno Célleri (UT), From Traditional to Interactive Playspaces: Automatic Analysis of Player Behavior in the Interactive Tag Playground.
22. Grace Lewis (VU), Software Architecture Strategies for Cyber-Foraging Systems.

23. Fei Cai (UVA), Query Auto Completion in Information Retrieval.

24. Brend Wanders (UT), Repurposing and Probabilistic Integration of Data; An Iterative and data model independent approach.

25. Julia Kiseleva (TU/e), Using Contextual Information to Understand Searching and Browsing Behavior.

26. Dilhan Thilakarathne (VU), In or Out of Control: Exploring Computational Models to Study the Role of $\mathrm{Hu}-$ man Awareness and Control in Behavioural Choices, with Applications in Aviation and Energy Management Domains.

27. Wen Li (TUD), Understanding Geospatial Information on Social Media.

28. Mingxin Zhang (TUD), Large-scale Agent-based Social Simulation - A study on epidemic prediction and control.

29. Nicolas Höning (TUD), Peak reduction in decentralised electricity systems - Markets and prices for flexible planning.

30. Ruud Mattheij (UvT), The Eyes Have It.

31. Mohammad Khelghati (UT), Deep web content monitoring. 
32. Eelco Vriezekolk (UT), Assessing Telecommunication Service Availability Risks for Crisis Organisations.

33. Peter Bloem (UVA), Single Sample Statistics, exercises in learning from just one example.

34. Dennis Schunselaar (TUE), Configurable Process Trees: Elicitation, Analysis, and Enactment.

35. Zhaochun Ren (UVA), Monitoring Social Media: Summarization, Classification and Recommendation.

36. Daphne Karreman (UT), Beyond R2D2: The design of nonverbal interaction behavior optimized for robot-specific morphologies.

37. Giovanni Sileno (UvA), Aligning Law and Action - a conceptual and computational inquiry.

38. Andrea Minuto (UT), Materials that Matter - Smart Materials meet Art \& Interaction Design.

39. Merijn Bruijnes (UT), Believable Suspect Agents; Response and Interpersonal Style Selection for an Artificial Suspect.

40. Christian Detweiler (TUD), Accounting for Values in Design.

41. Thomas King (TUD), Governing Governance: A Formal Framework for Analysing Institutional Design and Enactment Governance.
42. Spyros Martzoukos (UVA), Combinatorial and Compositional Aspects of Bilingual Aligned Corpora.

43. Saskia Koldijk (RUN), ContextAware Support for Stress SelfManagement: From Theory to Practice.

44. Thibault Sellam (UVA), Automatic Assistants for Database Exploration.

45. Bram van de Laar (UT), Experiencing Brain-Computer Interface Control.

46. Jorge Gallego Perez (UT), Robots to Make you Happy.

47. Christina Weber (UL), Real-time foresight - Preparedness for dynamic innovation networks.

48. Tanja Buttler (TUD), Collecting Lessons Learned.

49. Gleb Polevoy (TUD), Participation and Interaction in Projects. A GameTheoretic Analysis.

50. Yan Wang (UVT), The Bridge of Dreams: Towards a Method for Operational Performance Alignment in IT-enabled Service Supply Chains.

\section{7}

1. Jan-Jaap Oerlemans (UL), Investigating Cybercrime.

2. Sjoerd Timmer (UU), Designing and Understanding Forensic Bayesian Networks using Argumentation. 
3. Daniël Harold Telgen (UU), Grid Manufacturing; A CyberPhysical Approach with Autonomous Products and Reconfigurable Manufacturing Machines.

4. Mrunal Gawade (CWI), Multi-core Parallelism in a Column-store.

5. Mahdieh Shadi (UVA), Collaboration Behavior.

6. Damir Vandic (EUR), Intelligent Information Systems for Web Product Search.

7. Roel Bertens (UU), Insight in Information: from Abstract to Anomaly.

8. Rob Konijn (VU), Detecting Interesting Differences:Data Mining in Health Insurance Data using Outlier Detection and Subgroup Discovery.

9. Dong Nguyen (UT), Text as Social and Cultural Data: A Computational Perspective on Variation in Text.

10. Robby van Delden (UT), (Steering) Interactive Play Behavior.

11. Florian Kunneman (RUN), Modelling patterns of time and emotion in Twitter \#anticipointment.

12. Sander Leemans (TUE), Robust Process Mining with Guarantees.

13. Gijs Huisman (UT), Social Touch Technology - Extending the reach of social touch through haptic technology.
14. Shoshannah Tekofsky (UvT), You Are Who You Play You Are: Modelling Player Traits from Video Game Behavior.

15. Peter Berck (RUN), Memory-Based Text Correction.

16. Aleksandr Chuklin (UVA), Understanding and Modeling Users of Modern Search Engines.

17. Daniel Dimov (UL), Crowdsourced Online Dispute Resolution.

18. Ridho Reinanda (UVA), Entity Associations for Search.

19. Jeroen Vuurens (UT), Proximity of Terms, Texts and Semantic Vectors in Information Retrieval.

20. Mohammadbashir Sedighi (TUD), Fostering Engagement in Knowledge Sharing: The Role of Perceived Benefits, Costs and Visibility.

21. Jeroen Linssen (UT), Meta Matters in Interactive Storytelling and Serious Gaming (A Play on Worlds).

22. Sara Magliacane (VU), Logics for causal inference under uncertainty.

23. David Graus (UVA), Entities of Interest — Discovery in Digital Traces.

24. Chang Wang (TUD), Use of Affordances for Efficient Robot Learning.

25. Veruska Zamborlini (VU), Knowledge Representation for Clinical Guidelines, with applications to Multimorbidity Analysis and Literature Search. 
26. Merel Jung (UT), Socially intelligent robots that understand and respond to human touch.

27. Michiel Joosse (UT), Investigating Positioning and Gaze Behaviors of Social Robots: People's Preferences, Perceptions and Behaviors.

28. John Klein (VU), Architecture Practices for Complex Contexts.

29. Adel Alhuraibi (UvT), From ITBusinessStrategic Alignment to Performance: A Moderated Mediation Model of Social Innovation, and Enterprise Governance of IT".

30. Wilma Latuny (UvT), The Power of Facial Expressions.

31. Ben Ruijl (UL), Advances in computational methods for QFT calculations.

32. Thaer Samar (RUN), Access to and Retrievability of Content in Web Archives.

33. Brigit van Loggem (OU), Towards a Design Rationale for Software Documentation: A Model of ComputerMediated Activity.

34. Maren Scheffel (OU), The Evaluation Framework for Learning Analytics.

35. Martine de Vos (VU), Interpreting natural science spreadsheets.

36. Yuanhao Guo (UL), Shape Analysis for Phenotype Characterisation from High-throughput Imaging.
37. Alejandro Montes Garcia (TUE), WiBAF: A Within Browser Adaptation Framework that Enables Control over Privacy.

38. Alex Kayal (TUD), Normative Social Applications.

39. Sara Ahmadi (RUN), Exploiting properties of the human auditory system and compressive sensing methods to increase noise robustness in ASR.

40. Altaf Hussain Abro (VUA), Steer your Mind: Computational Exploration of Human Control in Relation to Emotions, Desires and Social Support For applications in human-aware support systems.

41. Adnan Manzoor (VUA), Minding a Healthy Lifestyle: An Exploration of Mental Processes and a Smart Environment to Provide Support for a Healthy Lifestyle.

42. Elena Sokolova (RUN), Causal discovery from mixed and missing data with applications on ADHD datasets.

43. Maaike de Boer (RUN), Semantic Mapping in Video Retrieval.

44. Garm Lucassen (UU), Understanding User Stories - Computational Linguistics in Agile Requirements Engineering.

45. Bas Testerink (UU), Decentralized Runtime Norm Enforcement.

46. Jan Schneider (OU), Sensor-based Learning Support. 
47. Jie Yang (TUD), Crowd Knowledge Creation Acceleration.

48. Angel Suarez (OU), Collaborative inquiry-based learning.

\section{8}

1. Han van der Aa (VUA), Comparing and Aligning Process Representations.

2. Felix Mannhardt (TUE), Multiperspective Process Mining.

3. Steven Bosems (UT), Causal Models For Well-Being: Knowledge Modeling, Model-Driven Development of Context-Aware Applications, and Behavior Prediction.

4. Jordan Janeiro (TUD), Flexible Coordination Support for Diagnosis Teams in Data-Centric Engineering Tasks.

5. Hugo Huurdeman (UVA), Supporting the Complex Dynamics of the Information Seeking Process.

6. Dan Ionita (UT), Model-Driven Information Security Risk Assessment of Socio-Technical Systems.
7. Jieting Luo (UU), A formal account of opportunism in multi-agent systems.

8. Rick Smetsers (RUN), Advances in Model Learning for Software Systems.

9. Xu Xie (TUD), Data Assimilation in Discrete Event Simulations.

10. Julia S. Mollee (VU), Moving forward: supporting physical activity behavior change through intelligent technology.

11. Mahdi Sargolzaei (UVA), Enabling Framework for Service-oriented Collaborative Networks.

12. Xixi Lu (TUE), Using behavioral context in process mining.

13. Seyed Amin Tabatabaei (VU), Computing a Sustainable Future; Exploring the added value of computational models for increasing the use of renewable energy in the residential sector.

14. Bart Joosten (UVT), Detecting Social Signals with Spatiotemporal Gabor Filters.

15. Naser Davarzani (UM), Biomarker Discovery in Heart Failure. 